\title{
Hepatitis B Virus Genotypes in Latvia
}

\author{
Irina Sominskaya ${ }^{1}$, Maria Mihailova ${ }^{1}$, Juris Jansons ${ }^{*}, 1$, Diana Legzdina ${ }^{1}$, Gunita Sudmale ${ }^{1}$, \\ Paul Pumpens ${ }^{1}$, Frida Arsha ${ }^{2}$, Irena Davidjuka ${ }^{2}$, Jazeps Keish ${ }^{2}$, Valentina Sondore ${ }^{2}$, \\ Baiba Rozentale $^{2}$ and Ludmila Viksna ${ }^{2}$
}

${ }^{1}$ Latvian Biomedical Research and Study Centre, Ratsupites 1, Riga LV-1067, Latvia

${ }^{2}$ Infectology Center of Latvia, Linezera 3, Riga LV-1006, Latvia

\begin{abstract}
The aim of this study was to investigate the properties of HBV genomes isolated from patients with acute and chronic hepatitis, liver cirrhosis, and hepatic coma admitted to the Infectology Center of Latvia. HBV genotypes and HBsAg subtypes were determined by direct S-gene sequencing. Genotypes D $(72.17 \%)$ and A $(27.96 \%)$ were predominant. Only one case $(0.87 \%)$ of HBV genotype E was found. Prevalence of genotype D over genotype A was more strongly pronounced in isolates from patients with acute hepatitis than from patients with chronic hepatitis- $74 \%$ $24 \%$ and $68 \%-32 \%$, respectively. Number of previously described and some new mutations (Ser21 Thr in preC region, Tyr134Lys, Phe134Leu, Ile110Met, Thr118Ile and Ser136Tyr in S-gene) were discovered by direct sequencing of PCR fragments corresponding to preS/S and preC/C regions and to a fragment of the $\mathrm{X}$ gene of $\mathrm{HBV}$ genome. The most frequently mutations were found in the region of the basal core promoter, preC and major immunodominant region of core protein and domain $a$ of S protein. In general the rate of mutations discovered in chronic patients was just 1,23 times higher then in patients with acute hepatitis, at the same time the ratio for the hot-spot preC 1896 stop mutation was higher and reached 1,81. A possible role of discovered mutations in the HBV pathogenesis is discussed. The findings are relevant to diagnosis and prognosis of $\mathrm{HBV}$-induced liver disease.
\end{abstract}

Keywords: Hepatitis B virus, genotype, subtype, variant, mutation.

\section{INTRODUCTION}

The clinical course of hepatitis B virus (HBV) infection varies from self-limited and asymptomatic acute to fulminant hepatitis. A definite population of patients with acute HBV infection (AH) develops chronic liver disease: chronic hepatitis $(\mathrm{CH})$, liver cirrhosis (LC), and hepatocellular carcinoma (HCC). HBV is classified into 8 genotypes from A to $\mathrm{H}$ based on more than $8 \%$ sequence divergence of the entire genome sequences [1-3]. The HBV genotypes appear to be associated with particular geographic distribution, ethnicity, and possibly diagnoses and clinical outcome of the liver disease. At first, the HBV genotypes have distinct geographical distributions, e.g. A - Northwest Europe, North America, and Central Africa; D - Mediterranean area, Middle East, and India; E - sub-Saharan Africa. The HBV genotypes are divided into clusters or subgenotypes with 4 to $8 \%$ sequence divergence. Existence of two subgenotypes within the genotype A is described for South Africa [4]. These subgenotypes are assigned as A1 and A2 and the major difference between them is associated with to amino acid (aa) residues 207 and 209 in the HBs protein S (Asn/ Leu and Ser/Val substitutions for A1 and A2, respectively). It has been pointed out that European HBV strains belong to the subgenotype A2, whereas Afro-Asian strains belong to the subgenotype A1. A subgenotype A3, found in Cameroon,

*Address correspondence to this author at the Latvian Biomedical Research and Study Centre, Ratsupites 1, Riga, LV-1067, Latvia; Tel: 371-7808212; Fax: 371-7442407; E-mail jansons@biomed.lu.lv is a recombinant of the genotypes $\mathrm{A}$ and $\mathrm{E}$ [5]. The most widespread in the world is the genotype $\mathrm{D}$. This genotype is divided into four subgenotypes D1 to D4. The geographic distribution of these subgenotypes is less restricted than that for the genotype A, although the strains from the Middle East mainly belong to D1, from South Africa and Alaska to D3, and from Oceania and Somalia to D4 [6]. None of these subgenotypes is characterized with specific aa substitutions.

Traditionally, HBV is classified into 9 hepatitis B surface antigen (HBsAg) subtypes: $a d w 2, a d w 4, a d r q^{+}, a d r q^{-}, a y w 1$, ayw2, ayw3, ayw4 and ayr according to the phenotypic expression of the HBs-encoding part of the gene $\mathrm{S}$ of different HBV genotypes [7]. Epidemiological studies show that the prevalence of these subtypes also varies in different parts of the world and that they probably have a different clinical significance [8,9]. The $a d w 2$ and aywl subtypes usually belong to the genotype $\mathrm{A}$, whereas the ayw2, ayw3, ayw4 and $a d w 3$ subtypes belong to the genotype D. The ayw4 subtype appears in the genotype $\mathrm{E}$.

Despite the introduction of anti-HBV vaccination in 1997 and constant improvement of medical care in Latvia, the number of hepatitis B cases remains high. In previous papers we described appearance of HBV genotypes in Latvia $[10,11]$ and their distribution in two Latvian clinics: the Kidney transplantation Centre and the Hematology Unit of the Children's Clinical University Hospital in Riga [12,13]. Due to the specific nature of the clinics obtained data represented exclusively the HBV genome distribution for different sorts of chronic HBV infection. 
Table 1. Distribution of Patients Admitted to Study by Sex, Age, Gender and Major HBV and HCV Markers

\begin{tabular}{|c|c|c|c|c|c|c|c|}
\hline Desase & Total & Sex & Number & Age & HBV DNA + & HBV DNA - & a-HCV + \\
\hline Acute hepatitis & 89 & $\mathrm{f}$ & 42 & $44.22 \pm 18.26$ & 40 & 2 & 3 \\
\hline \multirow{2}{*}{ Chronic hepatitis } & \multirow{2}{*}{33} & $\mathrm{~m}$ & 24 & $40.91 \pm 17.65$ & 19 & 5 & 7 \\
\hline & & $\mathrm{f}$ & 9 & $36.00 \pm 19.87$ & 6 & 3 & 0 \\
\hline Other liver damages & 9 & $\mathrm{~m}$ & 6 & $27.67 \pm 2.89$ & 5 & 1 & 1 \\
\hline \multirow{2}{*}{ Total } & \multirow{2}{*}{131} & $\mathrm{~m}$ & 77 & $35.69 \pm 17.08$ & 66 & 11 & 16 \\
\hline & & $\mathrm{f}$ & 54 & $44.25 \pm 18.92$ & 49 & 5 & 3 \\
\hline
\end{tabular}

The aim of this study is to evaluate a present epidemiological distribution of the HBV genotypes, subgenotypes, and subtypes among patients of the Infectology Center of Latvia (ICL), a major medical institution in this field in Latvia, whose main specialization is acute HBV infection. We present here the appearance of mutations within the HBV genome regions EnhII/BCP/X-preC/C and $\mathrm{preS} / \mathrm{S}$ against the HBV genome prototypes. Obtained results shows that the distribution of the HBV genotypes in Latvia remains the same for all kinds of HBV-induced liver disease patients and diagnoses, and the profile of the medical institutions involved. More than 20-years long persistence of areaspecific HBV strains with preserved "old" mutations reveals a clear predominance of "local" HBV genotypes, despite the rapid development and growing migration rate in Latvia.

\section{MATERIALS AND METHODS}

\section{Patients}

A total of 131 (54 female and 77 male) consecutive patients of the ICL from December $1^{\text {st }} 1997$ to December $31^{\text {st }} 2006$ were admitted to the study. Diagnosis: $\mathrm{AH}, \mathrm{CH}$, LC, autoimmune hepatitis, and hepatic coma (Table 1). Diagnosis was based on traditional clinical, biochemical, and immunochemical hepatitis B marker tests. The mean age of the female and male patients was $44.25 \pm 18.92$ years and
$35.69 \pm 17.08$, respectively. State agency "Infectology Center of Latvia" and the Scientific Council of the Biomedical Research and Study Centre approved the protocol of this study and appropriate research permission was obtained from the independent Ethics Committee of Latvia. Informed consent to participate into this study was obtained from all included patients.

\section{Serological Tests}

The serum samples were stored at $-20{ }^{\circ} \mathrm{C}$ until assay was performed. All serum samples were tested for the presence of HBsAg (ETI-MAK-4, DiaSorin, Saluggia, Italy and Enzygnost HBsAg 5.0, Dade Behring, Marburg, Germany), total antibodies against hepatitis B core antigen (anti-HBc) (ETI-AB-COREK-2, DiaSorin, Saluggia, Italy), as well as for anti-HBc IgM, and anti-HBs. HBsAg positive samples were tested for hepatitis B e antigen (HBe) (ETI-EBK-2, DiaSorin, Saluggia, Italy) and antibodies against it (anti$\mathrm{HBe}$ ) (ETI-AB-EBK, DiaSorin, Saluggia, Italy). The study protocol was in agreement with rules of Latvian Ethics Committee.

\section{PCR Amplification and Sequence Analysis}

HBV DNA was extracted from $50 \mu \mathrm{l}$ of serum with a commercially available DNA-RNA isolation kit based on

Table 2. PCR Primers Used in Study

\begin{tabular}{|c|c|c|c|}
\hline \multicolumn{2}{|c|}{ Region of Analysis } & \multirow{2}{*}{$\frac{\text { Primer }}{17 \mathrm{p}}$} & \multirow{2}{*}{$\frac{\text { Sequence }}{\text { 5' TTATTTACATACTCTTTGGAAGGC 3' }^{\prime}}$} \\
\hline nres $/ \mathrm{S}$ & $2750-813 \mathrm{nt}$ & & \\
\hline Proding & $2100010 \mathrm{se}$ & 8.15 & 5' AATGTATACCCAAAGACAG 3', \\
\hline \multirow{2}{*}{ gene S (nested) } & \multirow{2}{*}{$215-710 \mathrm{nt}$} & S1p & 5' TTGTTGACAAGAATCCTCACAATACC 3' \\
\hline & & $\mathrm{S} 2 \mathrm{p}$ & 5' GCCCTACGAACCACTGAACAAATGG 3' \\
\hline \multirow{2}{*}{$\mathrm{preC} / \mathrm{C}$} & \multirow{2}{*}{$1741-2516 \mathrm{nt}$} & M3 & 5' CTGGGAGGAGTTGGGGGA 3' \\
\hline & & p19 & 5' AGGTACTGTAGAGGAATAAAGCCC 3' \\
\hline \multirow{2}{*}{ geneC (nested) } & \multirow{2}{*}{$2045-2387 n t$} & $\mathrm{C} 1 "$ & 5' GTTCACCTCACCATACTGCACTCAGGC 3' \\
\hline & & $\mathrm{C} 2{ }^{\prime}$ & 5' GAGTTCTTCTTCTAGGGGACCTGCCTCG 3' \\
\hline \multirow{2}{*}{$\mathrm{BCP} /$ preC (nested) } & \multirow{2}{*}{$1741-2114 \mathrm{nt}$} & M3 & 5' CTGGGAGGAGTTGGGGGA 3' \\
\hline & & 2.364 & 5' CCCAGGTAGCTAGAGTCAT 3' \\
\hline \multirow{2}{*}{ gene $\mathrm{X}$} & \multirow{2}{*}{$1505-1825 \mathrm{nt}$} & $21 \mathrm{p}$ & 5' CGTTCCAGCCGACCACGGGGCGC 3' \\
\hline & & $2-\mathrm{Sp}$ & 5' AAAAAGTTGCATGGTGCTGG 3' \\
\hline
\end{tabular}


phenol/chloroform extraction (Lyteh, Moscow, Russia). $\mathrm{HBV}$ genome fragments carrying the whole $\mathrm{preS} / \mathrm{S}$, $\mathrm{preC} / \mathrm{C}$ regions, and a fragment of the $\mathrm{X}$ gene, which included the EnII/BCP region, were PCR-amplified with several primer sets (Table 2), as previously described [13]. When amplifycation of the whole fragments failed, we used nested PCR with inner primers. Amplified HBV DNA fragments were sequenced in both directions directly using the ABI Prism Dye Terminator Cycle Sequencing Ready Reaction (Applied Biosystems, Foster City, Calif., USA) and electrophoregrams were obtained on an ABI Prism 377 sequencer (Applied Biosystems). In all cases PCR primers were used as sequencing primers. For sequencing of the complementary strand of the HBV preS region, an additional primer p14 - 5' CTGTAA CACGAGCAGGGGTCCTAG 3' was used [14]. The sequences were edited manually with the BioEdit Sequence Alignment Editor and subsequently aligned in the FASTA format (http://ngfnblast.gbf.de/docs/fasta.html). The phylogenetic tree was constructed by the MEGA program using the UPGMA algorithm.

\section{RESULTS AND DISCUSSION}

Sera of patients were divided into three groups: first group from 89 patients with $\mathrm{AH}$, second one from 33 patients with $\mathrm{CH}$, and third one from 9 patients with $\mathrm{LC}$, autoimmune hepatitis, hepatic coma, and fulminant hepatitis (FH). The last group was named "other liver damage" (Table 1). All AH sera patients were HBsAg and anti-HBc IgM positive. Sera of 19 patients contained anti-HCV antibodies. One serum of an AH patient contained anti-HAV antibodies and one of the $\mathrm{CH}$ patients was positive for HAV. Serological data concerning presence of $\mathrm{HBsAg}$ and $\mathrm{HBe} / \mathrm{anti}-\mathrm{HBe}$ patient status are listed in Table $\mathbf{3}$.

All samples were tested for HBV DNA by PCR; HBV DNA was detected in sera from 115 of the 131 patients (87.79\%), 16 samples were negative (12.21\%) (Table 1).

\section{Phylogenetic Analysis of HBV Genotypes}

The genotype of each HBV sequence was determined by comparison with sequences of known HBV genotypes A-H in the GenBank and EMBL nucleotide databases using the BLAST program. Phylogenetic tree was constructed for the HBV gene S (Fig. 1). Reference sequences, which are shown with their accession numbers, were obtained from the EMBL/GenBank database. Despite the multinational society existing in Latvia and increasing population mobility during recent years, we found only genotypes A (26.96\% of cases) and D ( $72.17 \%$ of cases) and only one case of a "foreign" genotype E $(0.87 \%)$ in the studied patients. Phylogenetic analysis clearly showed similarity between sequences of the genotype $\mathrm{A}$, whereas the genotype $\mathrm{D}$ sequences were more divergent. All genotype A isolates clustered together with A2 subgenotype sequences from the GenBank database and contained typical aa residue Ser at position 207 and Val at position 209. This means that the genotype A strains possess a rather low level of divergence and are "newcomers" to Latvia. The genotype D isolates had a more pronounced level of divergence and fell into three main clusters. The major cluster consisted of 48 isolates, which shared some similarity with the strains from the D2 subgenotype [6]. From this cluster, some subclusters were distinguished. Two isolates showed similarity with the strains from the D1

Table 3. Frequency of Specific Immunological Markers in Two Groups of Patients: AH, CH and other Liver Damages

\begin{tabular}{|c|c|c|c|c|c|}
\hline \multirow{2}{*}{ Disease } & \multirow{2}{*}{ Total } & HBsAg+ & HBsAg+ & \multirow{2}{*}{ HBsAg+ } & HBsA- \\
\cline { 3 - 5 } & & HBeAg+ & a-HBe + & & 1 \\
\hline \hline Acute hepatitis & 89 & 27 & 26 & 3 & 13 \\
\hline Chronic hepatitis & 33 & 16 & 8 & 3 \\
\hline Other liver damages & 9 & 3 & 2 & 3 \\
\hline
\end{tabular}

Table 4. Distribution of HVB Genotypes, Subgenotypes and HBsAg Subtypes between LIC Isolates. Number of Isolates with Mutations is Shown in Brackets

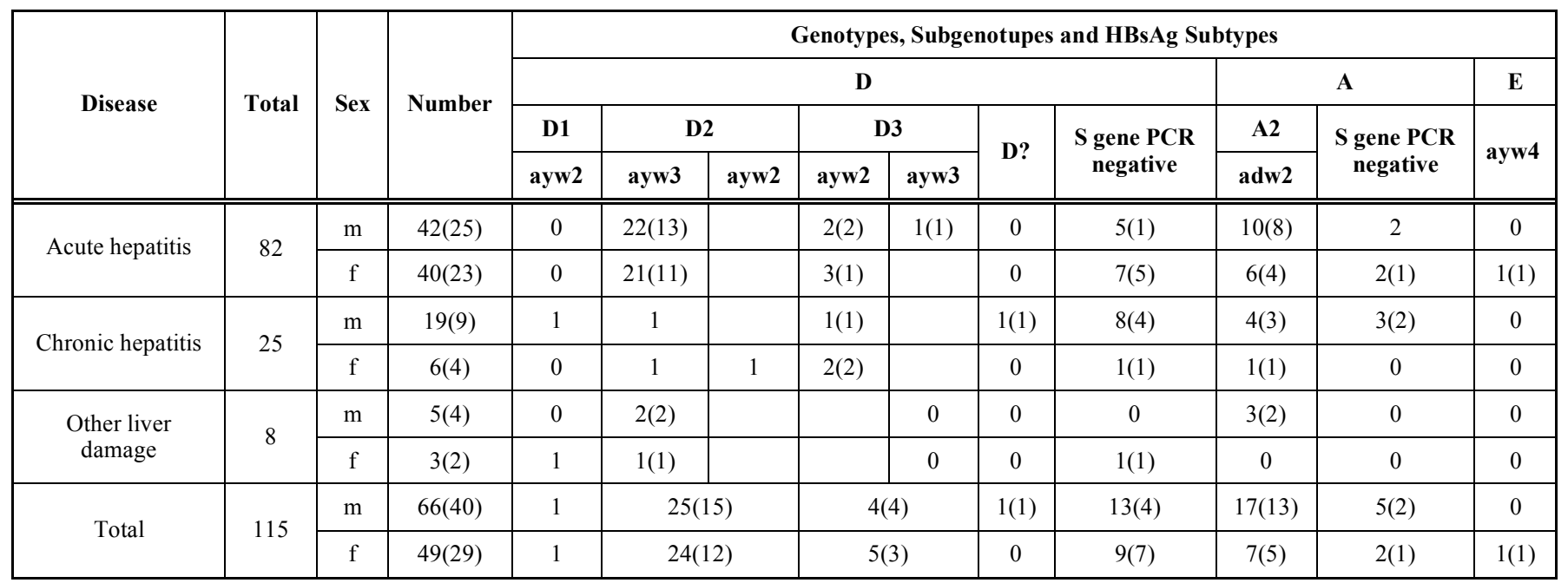




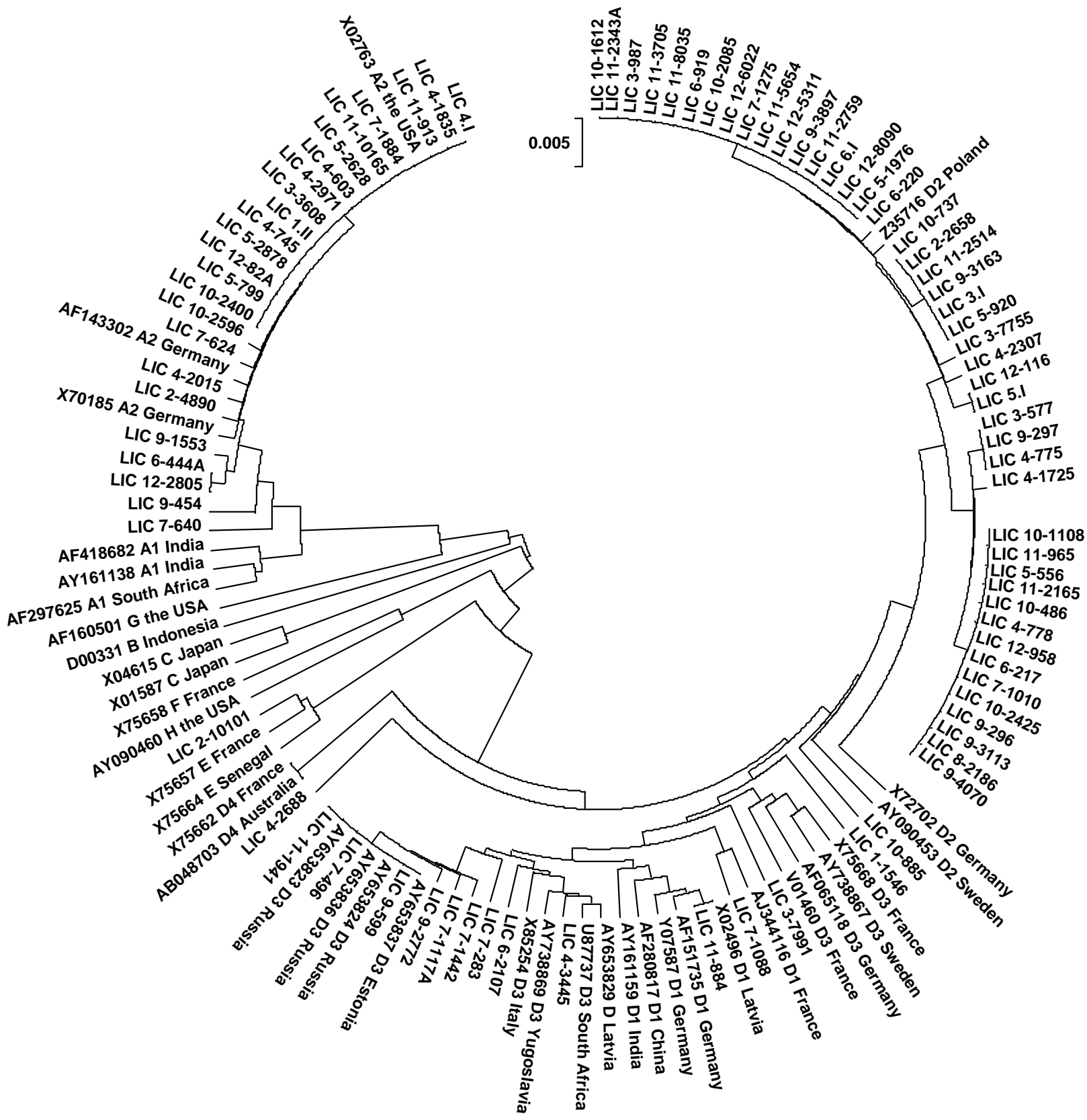

Fig. (1). The UPGMA phylogenetic tree based on sequences of fragments from HBV S-gene. Reference isolates are given specifying GeneBank access number, genotype and country of origin.

subgenotype. Nine isolates were similar to the subgenotype D3 genome.

Since monospecific subtype-determining antibodies were not available in Latvia, the HBV subtypes were determined by analysis of gene $\mathrm{S}$ sequences according to Magnius \& Norder [7]. $26.32 \%$ of our isolates belonged to the subtype $a d w 2$, and $10.53 \%$ and $47.37 \%$ represented the subtypes ayw2 and ayw3, respectively. In the case of one patient (1.32\%), LIC1-1546, it was impossible to define subtype, since mutation affected aa 127 of HBsAg, which is subtype- specific. In 22 cases $(13.16 \%)$ of the genotype D and 7 cases (3.95\%) of the genotype A, it was impossible to amplify the $\mathrm{S}$ region and determine subtypes, probably due to mutations within regions adjacent to primers (Table 4). All subgenotype A2 strains were of the $a d w 2$ subtype. All subgenotype D2 strains belonged to the ayw3 subtype. Two strains of the ayw2 subtype were of the D1 subgenotype. Eight strains with the subtype ayw2 and one of the ayw3 subtype belonged to the D3 subgenotype. The distribution of genotypes and subtypes within different groups of patients is listed in Table 4. 


\section{Sequence Variation in the HBV Genomes}

It is known that mutations appear in different regions of the HBV genome, and probably each stage of hepatitis B infection may be affected by the appearance of specific mutations [15]. Thorough analysis of major HBV regions $\mathrm{EnhII} / \mathrm{BCP} / \mathrm{X}-\mathrm{preC} / \mathrm{C}$ and $\mathrm{preS} / \mathrm{S}$ of three groups of patients

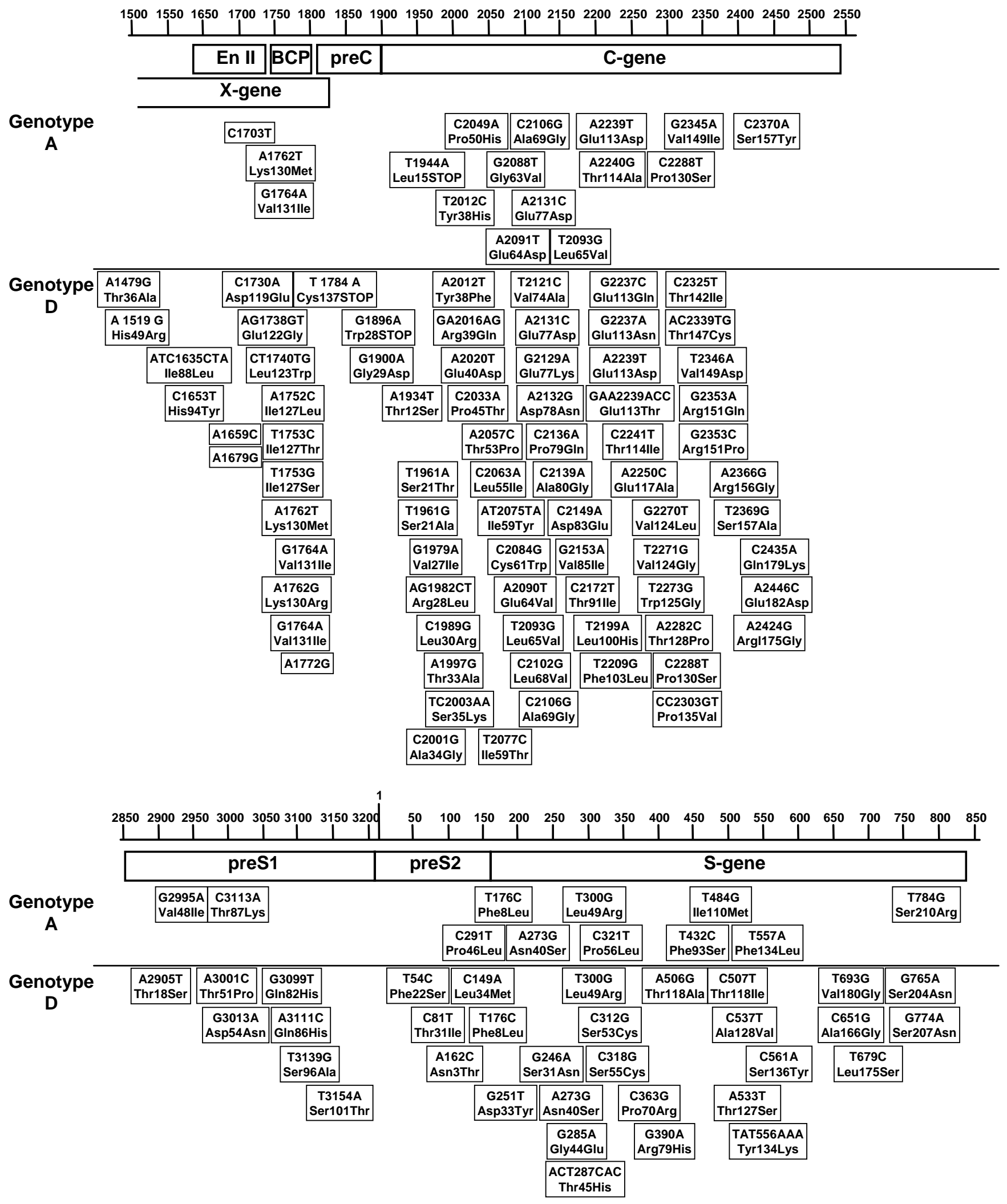

Fig. (2). Mutations discovered in tested HBV isolates. 
was performed with the intention of revealing an association of the appearing mutations with the clinical course of HBV infection. Sequences of analyzed HBV regions compared with reference HBV sequences are given in Appendix. Detailed analysis of sequenced HBV genes is presented in Figs. (2 and 3). After genotype- and subtype-specific mutations, it reveals also the presence of silent and missense mutations.

Examining the distribution of mutations within the groups of patients and HBV genotypes (Fig. 3), we may conclude that, in general, in groups of $\mathrm{CH}$ patients and patients with other liver damage, mutations occur more frequently than in groups of $\mathrm{AH}$ patients. On the other hand, strains of genotype $\mathrm{D}$ contain more mutations when compared to strains of genotype A.

The distribution of the mutations on the genome of HBV correlates well enough with arrangement of the functionally and immunologically important regions. On the diagram we can see some hot spots corresponding to $\mathrm{BCP}$, preC, MIR region in $\mathrm{C}$-gene, and domain $a$ in S-gene.

\section{Gene X/EnII/BCP}

The BCP mutations A1762T and G1764A, most frequently observed in $\mathrm{HBV}$-infected patients with $\mathrm{CH}, \mathrm{HCC}$, and $\mathrm{FH}$, were found in one $\mathrm{CH}$ and six $\mathrm{AH}$ cases. Additional mutations at $\mathrm{C} 1653 \mathrm{~T}$ were found in two $\mathrm{AH}$ cases and $\mathrm{T} 1753 \mathrm{C}$ in four $\mathrm{AH}$ and one $\mathrm{CH}$ case. These mutations may also be associated with the progression of liver disease to cirrhosis [16]. One AH isolate contained mutation at position $\mathrm{T} 1753 \mathrm{C}$ in conjunction with the G1764A mutation. One $\mathrm{CH}$ isolate contained $\mathrm{G} 1764 \mathrm{~T}$ and $\mathrm{C} 1765 \mathrm{G}$ mutations, which resulted in Val131Leu substitution in the gene $\mathrm{X}$, in conjunction with the T1752C and T1753G mutations, which led to Ile127Thr substitution. The C1653T mutation led to the His94Tyr substitution in the immunodominant epitope of the protein $\mathrm{X}$ [17]. The A1752C mutation in patients with $\mathrm{CH}$ was reported also in conjunction with the 1762 and 1764 mutations and appeared to be associated with liver damage [18]. We found this mutation in five cases, and in all cases it was independent of other mutations. Two $\mathrm{AH}$ samples contained aa substitution Ile88Leu within the protein X. One $\mathrm{AH}$ isolate possessed T1784A mutation, which led to conversion of Cys 137 to stop codon. Other individual AH isolates demonstrated A1479G mutation leading to the Thr36Ala substitution, A1519G mutation leading to His49Arg substitution, and C1730A mutations leading to Asp119Glu substitution. One AH isolate carried four mutations: AG1738GT and CT1740TG, which changed two aa residues: Glu122Gly and Leu123Trp.

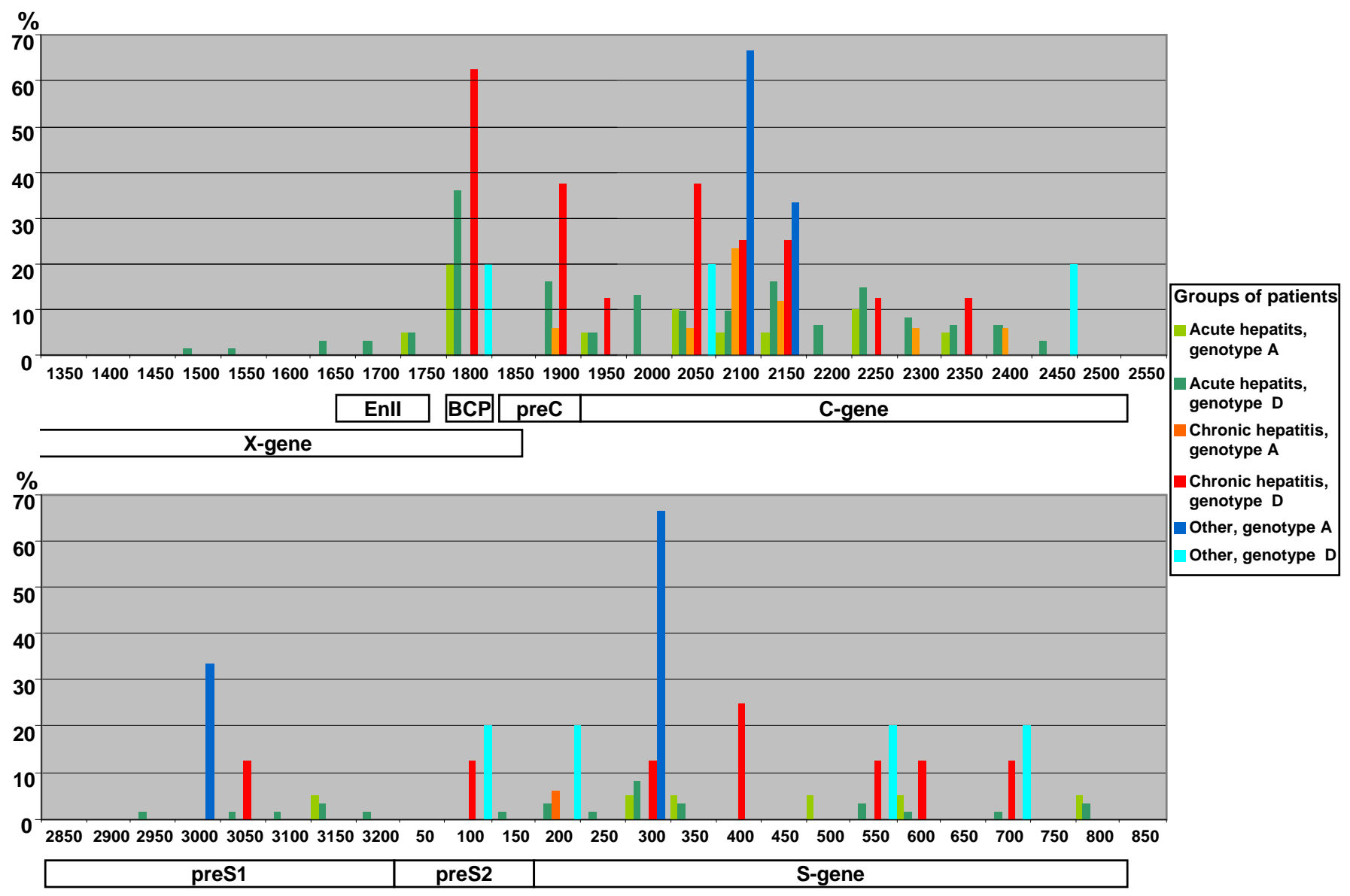

Fig. (3). Frequency of mutations discovered in HBV genome within groups of LIC patients and HBV genotypes. 
It is interesting that the hepatic coma isolate possessed only few mutations, but two of them were located within the EnhII region.

\section{PreC/C Gene}

The preC stop codon mutation G1896A leading to substitution of the preC Trp -28 to UGA stop codon was detected in eight $\mathrm{AH}$ and three $\mathrm{CH}$ isolates. Absence of HBeAg in all except one patients' sera confirmed the observation that such mutations abort $\mathrm{HBeAg}$ expression and cause $\mathrm{CH}$ disease $[19,20]$. The one $\mathrm{CH}$ and two $\mathrm{AH}$ isolates contained mutation at position $1900(\mathrm{G} \rightarrow \mathrm{A})$, which changed aa 29 of the preC (Gly29Asp).

We detected numerous mutations within the $\mathrm{HBc}$ region 84-101, which overlaps the CTL epitopes (Fig. 2). Such mutations accumulate usually during periods with frequent hepatitis exacerbation [21,22]. Numerous unique mutations were found in the region 48-60 (Fig. 2), which were described as typical for $\mathrm{FH}$ [23-25] and severe exacerbation hepatitis $\mathrm{B}$ patients [26,27]. We detected also mutations in previously described mutation clustering regions at aa 21-34 [28] and 31-49 [29].

In two $\mathrm{AH}$ isolates we found substitutions within the HBc CTL epitope 18-27: Ser21Ala and Ser21Thr, which were not described before. HBc CTL epitope 18-27 mutations Ser21Asn/Ala/Val were found earlier in HLA-A2positive $\mathrm{CH}$ patients [30]. In other HBc CTL epitopes: 63-71 and 141-149 [31], the following substitutions were found Gly63Val (twice in $\mathrm{AH}$ and $\mathrm{CH}$ patients), Glu64Asp (twice in $\mathrm{LC}$ and $\mathrm{CH}$ patients), and Glu64Val (once in $\mathrm{AH}$ patient). The Glu64Asp substitution was combined with two other mutations within the same epitope: Leu65Val and Ala69Gly. Mutations were found also within the C-terminal CTL epitope 141-149 (Fig. 2).

We found Pro130Ser substitution in two isolates: one $\mathrm{AH}$ and one $\mathrm{CH}$. The aa 130 belongs to both T helper and $\mathrm{B}$ cell epitopes, and the Pro130Ile/Thr/Ser substitutions could be associated with exacerbation of chronic hepatitis [32,33] and HCC [34]. Such substitutions were detected in patients with or without $\mathrm{HBeAg}$ [35]. In our case the $\mathrm{AH}$ patient had no HBeAg in his sera, whereas $\mathrm{CH}$ patient had it. The Arg151Gln substitution, which could be regarded as common, was found once as well as Arg151Pro substitution, both in $\mathrm{AH}$ patients. We found a set of mutations within the T helper epitope 50-69 and within the B cell epitope 74-83, previously detected in patients seroconverted from $\mathrm{HBe}$ to anti-HBe [36]: Thr53Pro/Ala was found in two cases in $\mathrm{CH}$ and $\mathrm{AH}$ patients with anti-HBe in sera. The $\mathrm{AH}$ patient sample contained also Cys61Trp substitution. The Glu77Lys/ Asp substitution was found in four AH patients, one of them was anti-HBe positive, another one was however $\mathrm{HBeAg}$ positive. The latter patient possessed also Asp78Asn substitution. The Pro79Gln mutation was found in one $\mathrm{AH}$ and two $\mathrm{CH}$ isolates, but the Ala80Gly substitution was found in an $\mathrm{AH}$ isolate, two of them were anti-HBe positive. A large number of mutations was found within the $\alpha 2 \mathrm{a}, \alpha 2 \mathrm{~b}$, and $\alpha 5$ helices of the HBc protein. The most frequent among them were: Tyr38His (two AH cases) and Tyr38Phe (one $\mathrm{AH}$ case), Glu40Asp (three AH and three $\mathrm{CH}$ cases), Glu113Gln (one $\mathrm{AH}$ and one $\mathrm{CH}$ case), Glu113Asp (four $\mathrm{AH}$ cases).
The Glu113Asn substitution was found once in $\mathrm{AH}$ isolate, Glu113Thr also once in AH isolate. The Thr114Ala/Ile and ValGlu124Leu substitutions were found in two AH cases.

In contrast to earlier observations [37], in all our studies we did not find any $\mathrm{HBc}$ deletion variants.

\section{PreS/S Gene}

Two mutations were found in the HBV preS1 region five times: G2995A that leads to Val48Ile substitution and G3001C - to Asp51His substitution. These mutations appeared in $a d w 2$ patients, three times in $\mathrm{AH}$ and twice in $\mathrm{CH}$ patients. Single G2995A mutation was found in an LC isolate. One $\mathrm{CH}$ isolate possessed $\mathrm{A} 3001 \mathrm{C}$ mutation leading to the Thr51Pro substitution. Besides this, the following individual mutations were observed: A2905T (Thr18Ser), G3013A (Asp54Asn), G3099T (Gln82His), A3111C (Gln86His), C3113A (Thr87Lys), T3139G (Ser96Ala), T3154A (Ser101Thr), all in AH isolates.

Only three mutations were found within the preS2 region: $\mathrm{T} 54 \mathrm{C}$ (Phe22Ser) in a $\mathrm{CH}$ isolate, $\mathrm{C} 81 \mathrm{~T}$ (Thr31Ile) in a coma patient and C149A (Leu34Met) in an AH patient.

It was documented that chronic HBV carriers and HCC patients frequently have mutations encompassing residues 29-53 [38] of the S region. We found some mutations within this region, but only Leu49Arg substitution was found twice, but in $\mathrm{AH}$ isolates. Within the site of hypervariability located at position 134 [39] we found the following substitutions: Tyr134Phe/Asn/Trp/Ser or Phe134Tyr depending of the HBsAg subtype. At this position we found some novel substitutions - Tyr134Lys in a $\mathrm{CH}$ patient and Phe134Leu in an $\mathrm{AH}$ patient. Novel substitutions were also observed at positions Ile110Met, Ser136Tyr, and Thr118Ile, all in $\mathrm{AH}$ patients. Only Thr118Ala substitution was reported earlier [40]. We found the Leu175Ser substitution in the hepatic coma patient, although this mutation appeared before only in combination with a Phe179Tyr substitution [41].

\section{HBV GENOTYPES IN LATVIA AND NEIGHBORING COUNTRIES}

The present study is the first epidemiological attempt examining the prevalence of the HBV genotypes A and D and their mutational status in the wide group of patients admitted to the Infectology Center of Latvia. Our findings reflect the overall genotype and subtype distribution in the Latvian population: $71.52 \%$ of genotype D and $27.81 \%$ of genotype A, $0.87 \%$ of genotype E; $23.18 \%$ of subtype $a d w 2$, $15.23 \%$ of subtype $a y w 2,39.07 \%$ of subtype $a y w 3,0.66 \%$ of subtype ayw4 and $1.99 a d w 3$. In one case, it was impossible to establish subtype due to unique Thr127Ser substitution at the subtype-specific residue, which has not been described previously. In our neighbor country, Poland, the genotype A is prevalent (74.1\%) [42], but the genotype D is on the second place $(20.7 \%)$. Almost all HBV genotype A cases are of the $a d w 2$ subtype. As to the genotype D similar to major subtypes found in Latvia, they are represented by ayw3 and ayw2 variants. In Estonia, another neighboring country [43], the situation is similar to Latvia, with predominance of the genotype $\mathrm{D}$ and prevalence of the 
subtypes $a y w 3$ and $a d w 2$. In contrast to Latvia, both neighboring countries show only a few cases of the ayw4 subtype. Data from another neighbor, Belarus, show also prevalence of the D genotype (87.5\%) over the A genotype $(12.5 \%)$ [44]. The most widespread within the D genotype in Belarus is the D2 subgenotype $(56.3 \%)$. In contrast to our data, the subgenotype D4 (3.1\%) was also found. Our neighbor to the East, the Russian Federation, is very large and proportion of HBV genotypes varies strongly depending on geographic location [45]. Thus, the genotype D comprises $85 \%$ among children in Moscow $[45,46]$. In Western Siberia, the genotype D constitutes even more than $96 \%$ of the total. In contrast to Latvia, the ayw2 subtype was clearly predominant over the ayw3 subtype [47]. Our data also support the postulate, that genotype D is predominant in the Mediterranean region, Southern and Eastern Europe, in such countries as Hungary [48], Italy: $73 \%$ of the genotype D, and $26 \%$ of the genotype A [49,50], and Spain: 63,2\% and $23.1 \%$, respectively [51,52]. In most Western European countries, such as Belgium 54\% of genotype A, and $31 \%$ of genotype D [53], the Netherlands $64 \%$, and $21 \%$ respectively [54] genotype A dominates.

\section{CONCLUSIONS}

- $\quad$ This study indicates that the genotype D is a predominant genotype among patients in Latvia, as in most of the neighboring countries The genotype $\mathrm{D}$ isolates are more heterogeneous than those of the genotype A.

- PreC/C mutants are prevalent among patients of Latvia. A never previously reported preC substitution Ser21Thr was found. At the same time we found no evidence for the existence of preC deletion variants.

- Comparison of the HBV sequences from the preS/S region with the strain of the same genotype revealed no deletions and relatively low number of mutations. At the same time we discovered $\mathrm{S}$ protein substitutions Tyr134Lys and Phe134Leu not previously described. Novel substitutions were observed also at positions Ile110Met, Thr118Ile and Ser136Tyr.

\section{ACKNOWLEDGEMENTS}

We thank Prof. Eva Stankevica and her group for oligonucleotide synthesis and automatic sequencing of $\mathrm{HBV}$ genome fragments, and Mrs. Viktorija Emeljanova for technical assistance.

This work was supported by National Program 10.0029.2 of the Latvian Council of Sciences, ERAF project 2DP/ 2.1.1.1.0/10/APIA/VIAA/164 and ESF project $1 \mathrm{DP} /$ 1.1.1.2.0/09/APIA/VIAA/150.

\section{Appendix}

Aligned nucleotide and protein sequences of different regions of Hepatitis B genome obtained during the study. Sequences are ranged by genotype and diagnosis of patients: $\mathrm{AH}$ - acute hepatitis, $\mathrm{CH}$ - chronic hepatitis, COMPL - other complications. As a reference sequences are given for genotype A - X02763, for genotype D - V01460 and X02496, for genotype E - X75657. Synonymous nucleotides are shown as dots.

Appendix Part A. Sequences of HBV region preC/C obtained during the study.

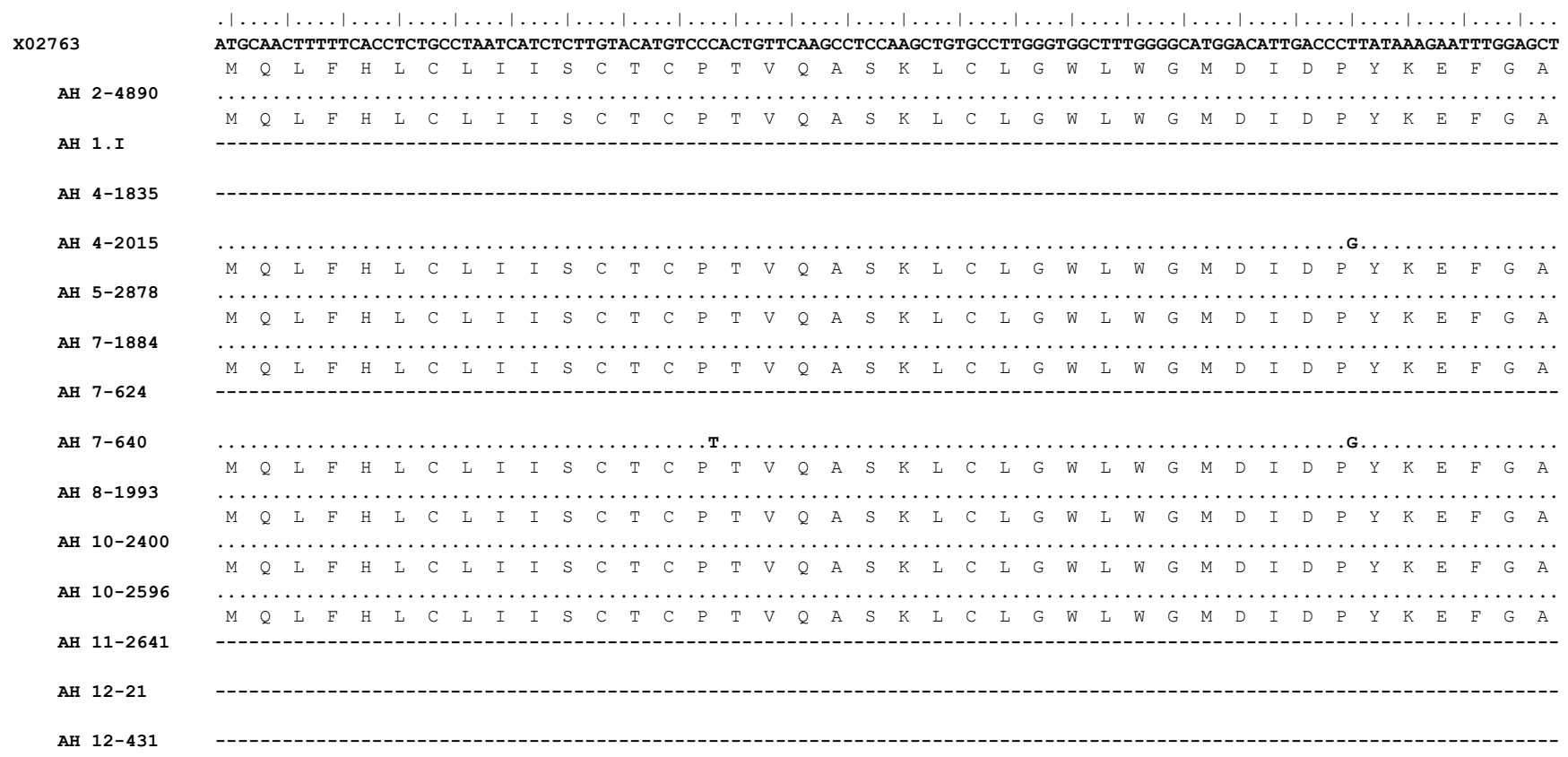




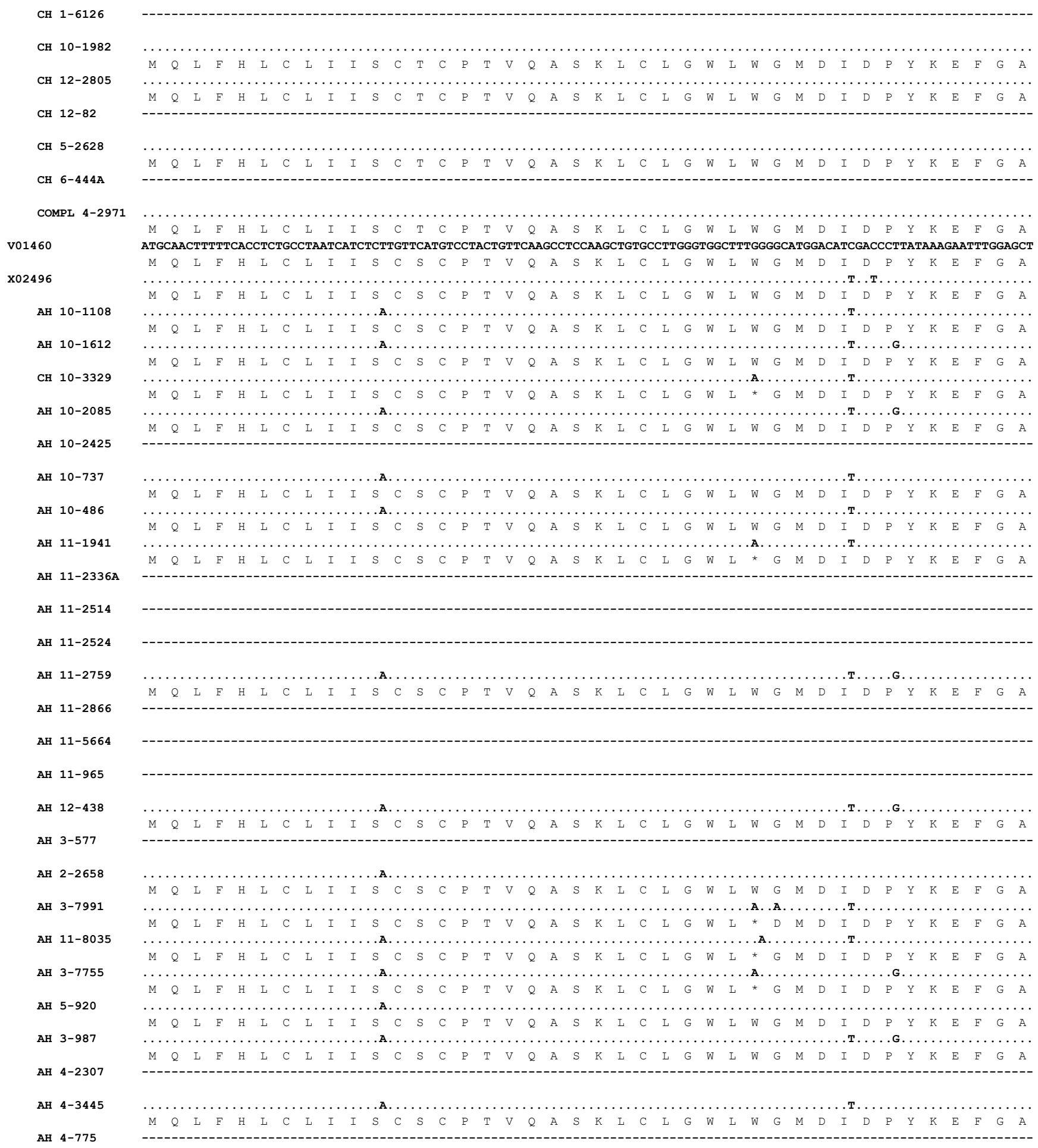


v01460

x02496

AH 4-778

AH 6.I

AH 5-556

AH $\quad 6-1289$

AH 6-217

AH $6-220$

AH 6-2394

AH 6-2616

AH 7-1010

AH 7-1275

AH 7-1440

AH 7-1442

AH 7-1501

AH 7-283

AH 7-3552

AH $8-1853$

AH $8-2186$

AH $9-2772$

AH 9-296

AH $9-3113$

AH $9-3163$

AH 9-4070

CH $1-1546$

CH 1-4936

CH $10-3335$

CH 11-2343A

CH 11-2866

CH 11-5664

CH 11-884

CH 12-2178

CH 12-958

CH 6-2625

CH 7-1117A

CH 7-2508

CH 9-599

CH $9-7181$

COMPL 11-3028

COMPL 11-3179

COMPL 4-1725

COMPL 7-2083

X75657

AH 2-10101

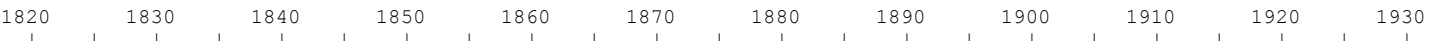
ATGCAACTTTTTCACCTCTGCCTAATCATCTCTTGTTCATGTCCTACTGTTCAAGCCTCCAAGCTGTGCCTTGGGTGGCTTTGGGGATGGACATCGACCCTTATAAAGA $\ldots \ldots|\ldots| \ldots$

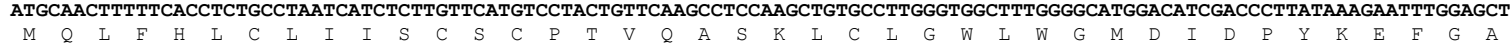

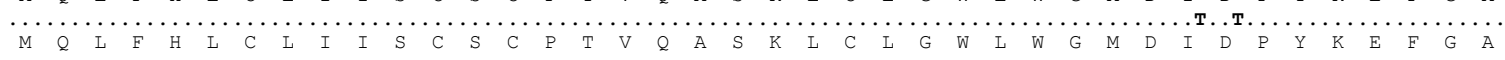

-

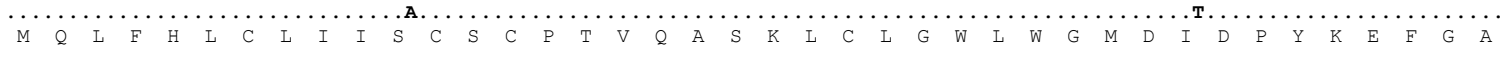

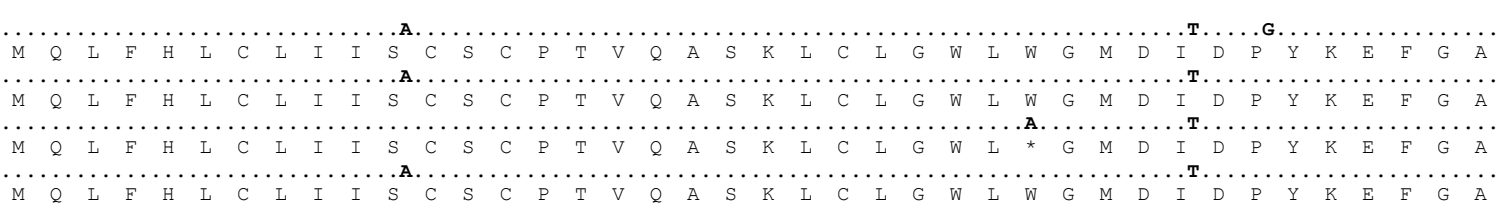

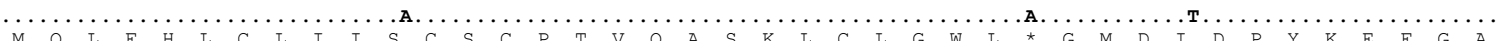

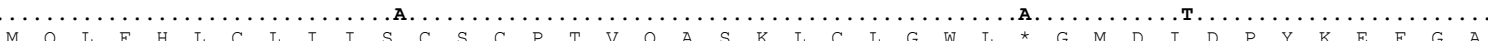

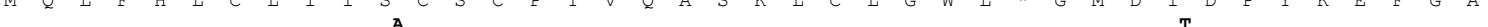

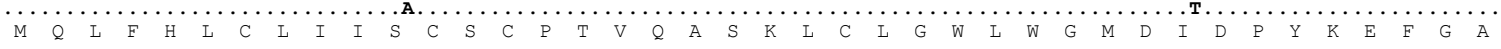

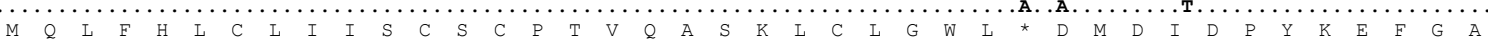

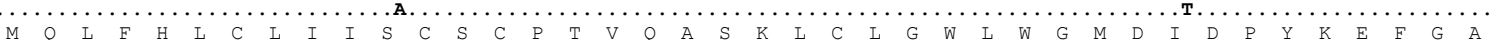

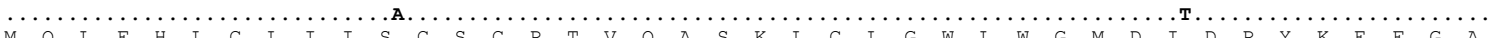
$\begin{array}{lllllllllllllllllll} & & & \end{array}$

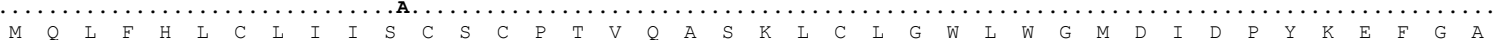

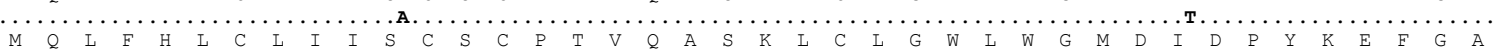

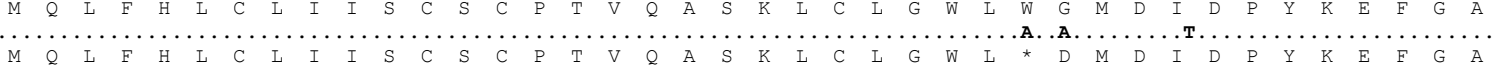

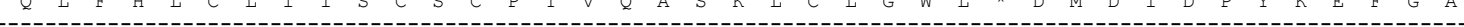

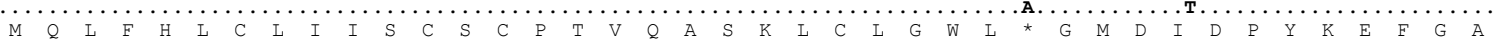

\section{(1)}

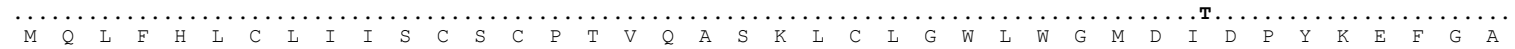

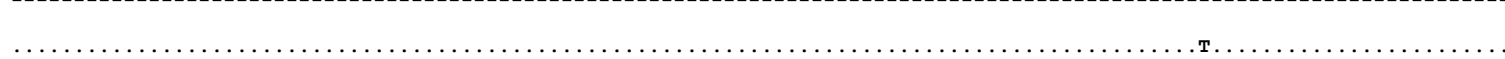

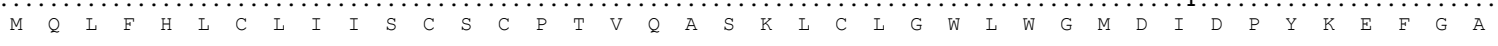

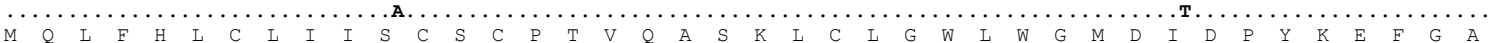

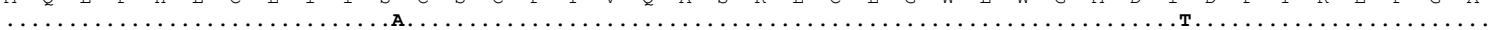

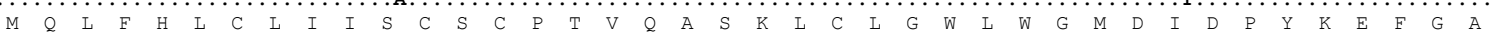

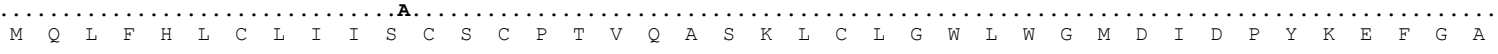

ATGCAACTTTTTCACСTCTGCCTAATCATCTCTTGTTCATGTCCTACTGTTCAAGCCTCCAAGCTGTGCCTTGGGTGGCTTTGGGGCATGGACATTGACCCTTATAAAGAATTTGGAGCT

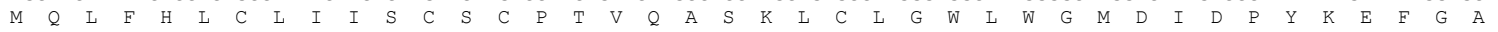

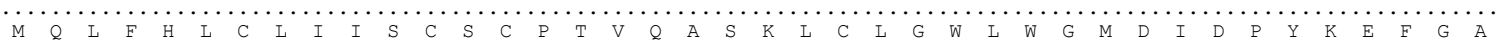


$\mathrm{x} 02763$

AH 2-4890

AH $1 . \mathrm{I}$

AH 4-1835

AH 4-2015

AH 5-2878

AH 7-1884

AH 7-624

AH $7-640$

AH 8-1993

AH $10-2400$

AH 10-2596

AH 11-2641

AH $12-21$

AH $12-431$

CH $1-6126$

CH 10-1982

CH 12-2805

CH 12-82

CH 5-2628

CH $6-444 \mathrm{~A}$

COMPL 4-2971

v01460

X02496

AH $10-1108$

AH 10-1612

CH $10-3329$

AH $10-2085$

AH $10-2425$

AH 10-737

AH 10-486

AH 11-1941

AH 11-2336A

AH 11-2514

AH 11-2524

AH 11-2759

AH 11-2866

AH 11-5664

AH 11-965

AH $\quad 12-438$

AH 3-577

AH 2-2658

AH 3-7991

AH 11-8035

AH 3-7755

AH 5-920

AH 3-987

AH 4-2307

AH 4-3445

AH 4-775

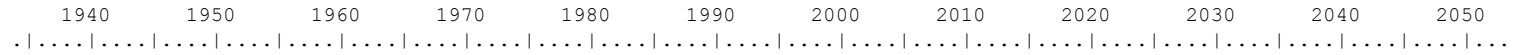

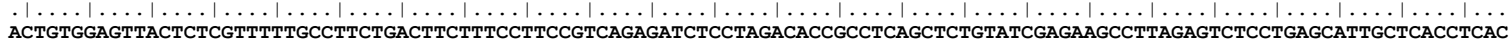

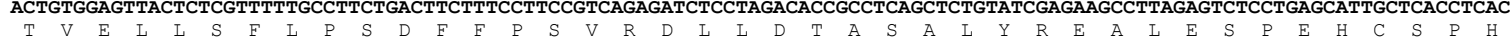
$T$
$T$ P

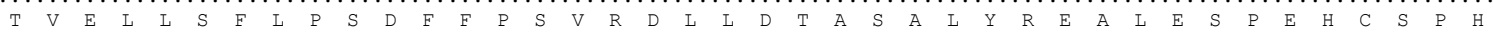

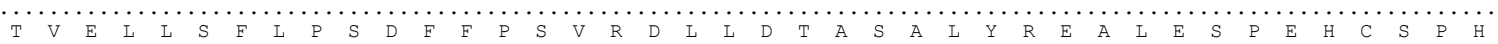

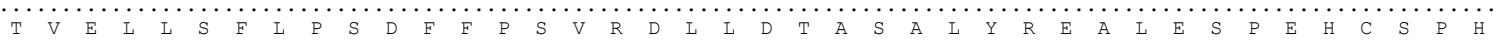

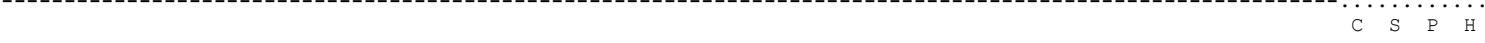

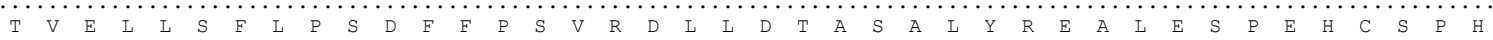

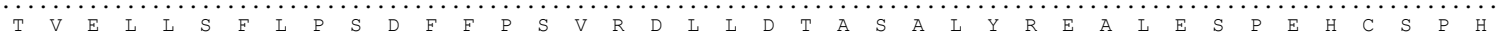

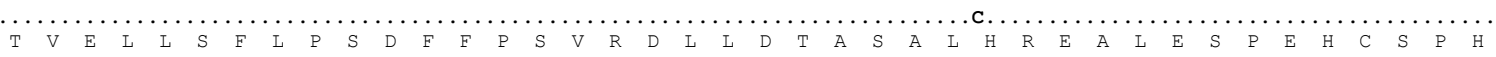

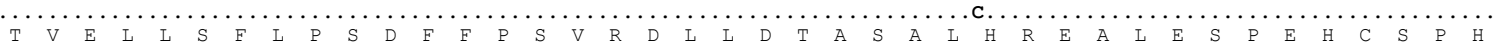

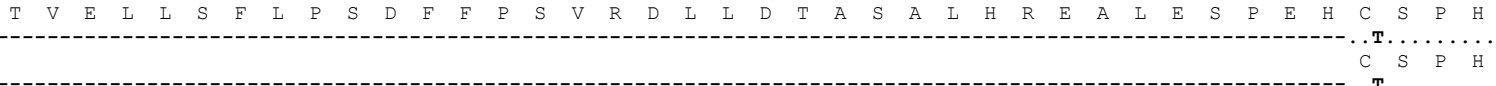
T. T. .т........ C $S$ P $\quad$ H C $\mathrm{S}$ P $\mathrm{P}$ H

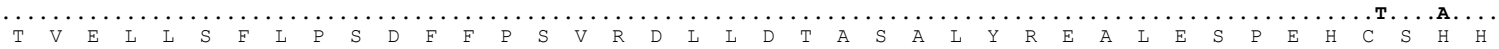

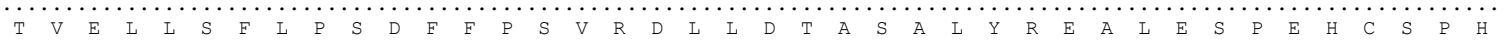

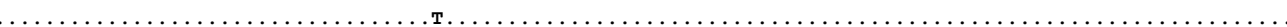

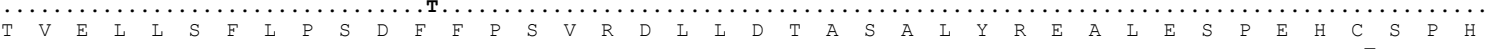

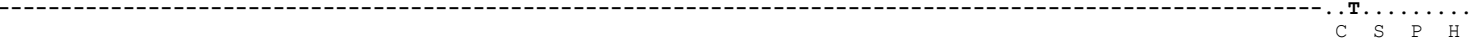

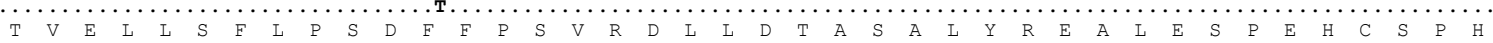
ACTGTGGAGTTACTCTCGTTTTTGCCTTCTGACTTCTTTCCTTCAGTACGAGATCTTCTAGATACCGCCTCAGCTCTGTATCGGGAAGCCTTAGAGTCTCCTGAGCATTGTTCACCTCAC

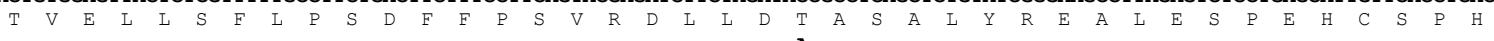

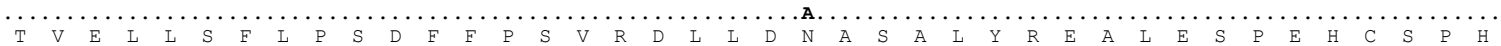

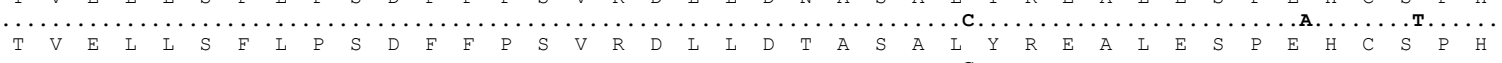

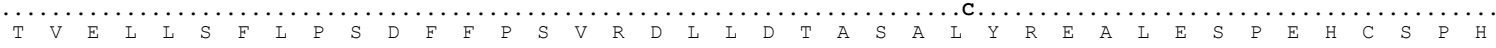

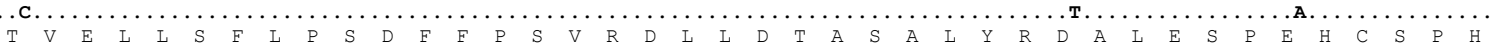

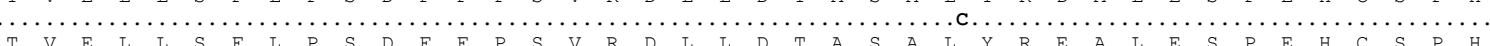
$\begin{array}{ccccccccccccccccccccccccccccccccccccccccccccc}T & V & E & L & L & S & F & L & P & S & D & F & F & P & S & V & R & D & L & L & D & T & A & S & A & L & Y & R & E & A & L & E & S & P & E & H & C & S & P & H \\ \end{array}$

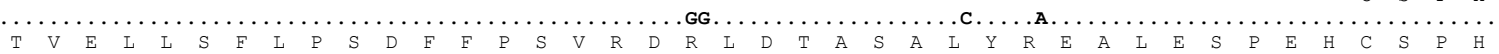

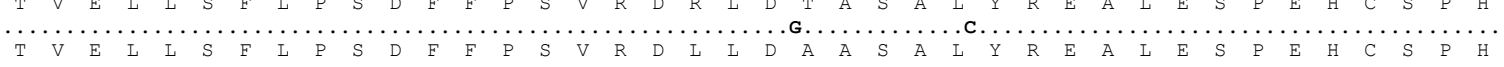

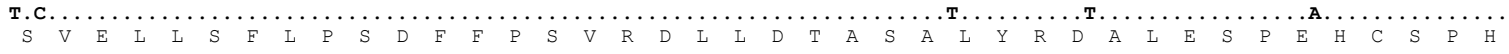
C $\quad$ S $P$ H C $\begin{array}{lll}\text { C } & \text { P } & \text { H }\end{array}$

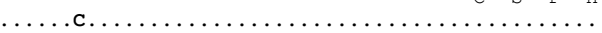

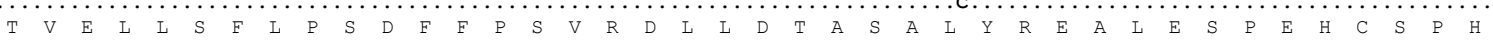
C $\quad$ S $P$ P C $\begin{array}{lll}\text { S } & \text { P } & \text { H }\end{array}$ C $\begin{array}{llll}\text { C } & P & \text { H }\end{array}$

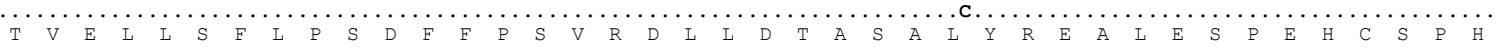

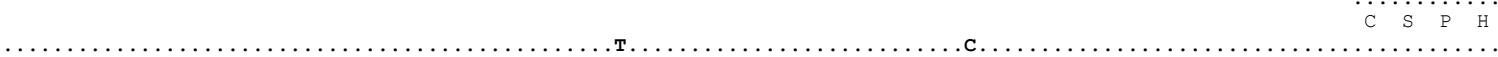

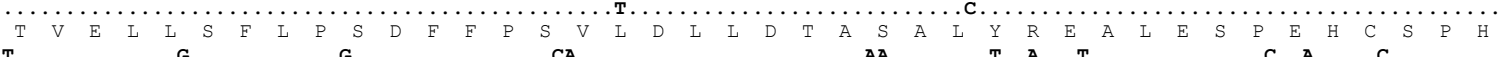

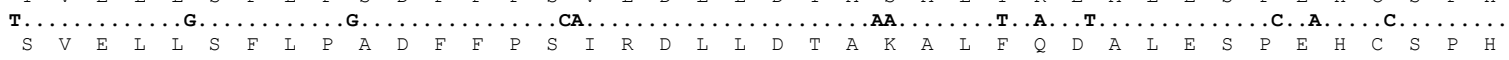

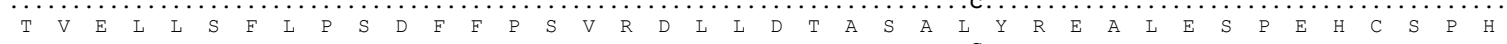

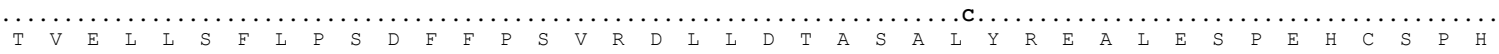
$M$
$T$

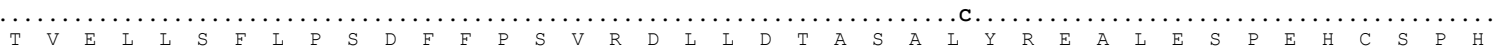
C $\underset{S}{\mathrm{~S}} \stackrel{\mathrm{P}}{\mathrm{H}}$

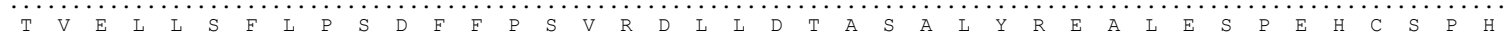
$\underset{\mathrm{C}}{\mathrm{S}} \mathrm{P}_{\mathrm{P}} \mathrm{H}_{\mathrm{H}}$ 
v01460

X02496

AH 4-778

AH 6.I

AH 5-556

AH $\quad 6-1289$

AH 6-217

AH $6-220$

AH 6-2394

AH 6-2616

AH 7-1010

AH 7-1275

AH 7-1440

AH 7-1442

AH 7-1501

AH 7-283

AH 7-3552

AH $8-1853$

AH $8-2186$

AH 9-2772

AH 9-296

AH $9-3113$

AH $9-3163$

AH 9-4070

CH 1-1546

CH 1-4936

CH 10-3335

CH 11-2343A

CH 11-2866

CH 11-5664

CH 11-884

CH 12-2178

CH 12-958

CH 6-2625

CH $7-1117 \mathrm{~A}$

CH 7-2508

CH 9-599

CH 9-7181

COMPL 11-3028

COMPL 11-3179 COMPL 4-1725

COMPL 7-2083

X75657

AH 2-1010

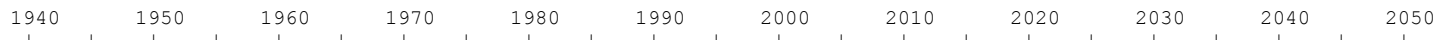
ACTGTGGAGTTACTCTCGTTTTTGCCTTCTGACTTCTTTCCTTCAGTACGAGATCTTCTAGATACCGCCTCAGCTCTGTATCGGGAGCCTTAGAGTCTCCTGAGCATTGTTCACCTCAC

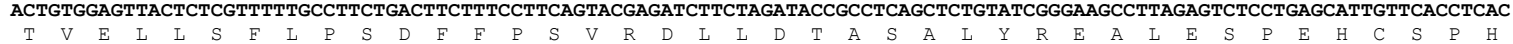

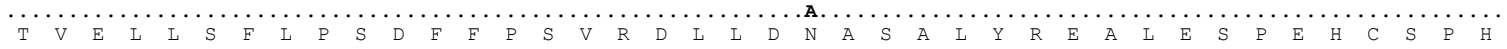

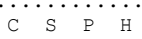
C $S$ P

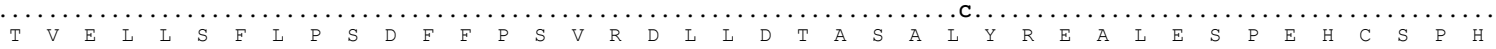
$\begin{array}{lll}\ldots & \ldots & \ldots \\ C & & \end{array}$ 作

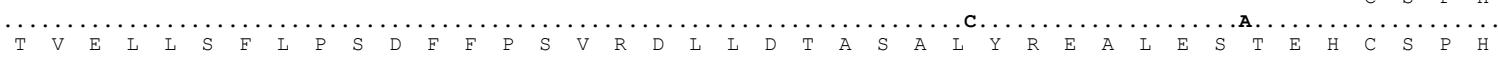

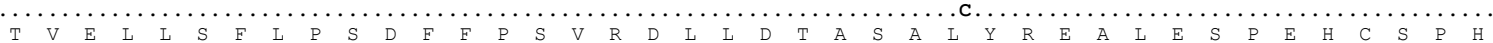

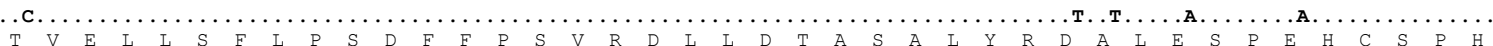

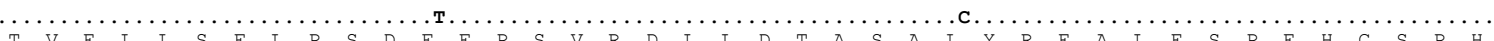

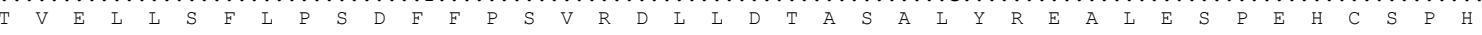
C $S$ P C $\begin{array}{llll}\text { C } & \text { P } & \text { H }\end{array}$ C $\begin{array}{lll}\text { C } & \text { P } & \text { H }\end{array}$

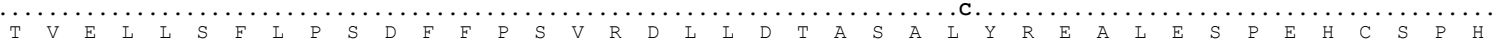
.

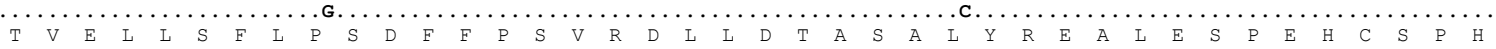

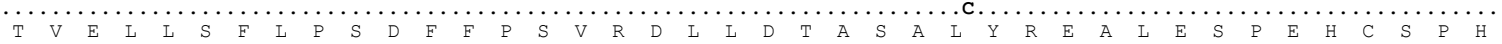

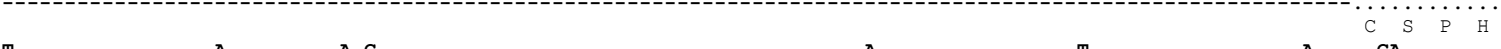

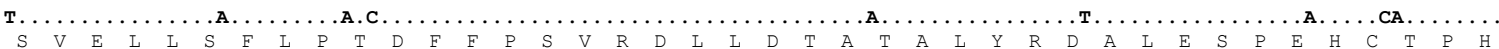

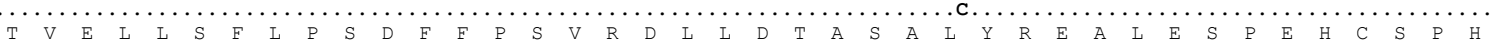

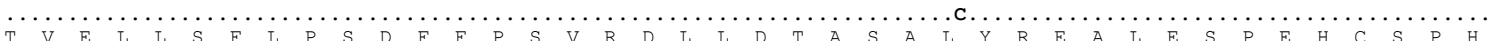

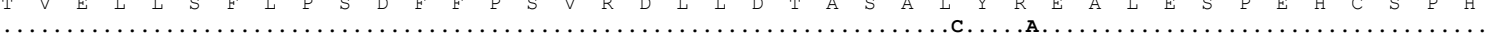

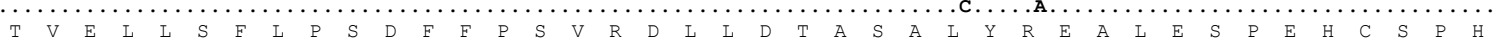

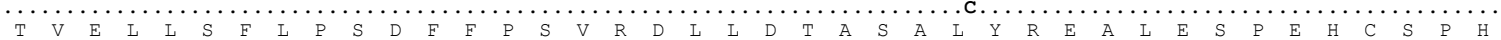
$T . C$.
$S$

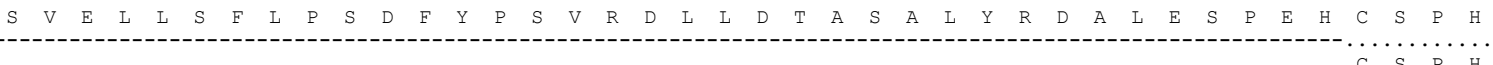

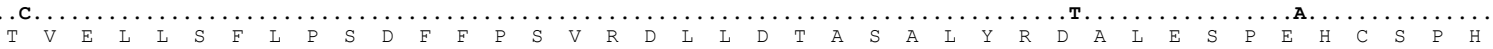
$\begin{array}{cccccccccccccccccccccccccccccccccccccccc}T & V & E & L & L & S & F & L & P & S & D & F & F & P & S & V & R & D & L & L & D & T & A & S & A & L & Y & R & D & A & L & E & S & P & E & H & C & S & P & H \\ -\end{array}$ ${ }_{C}{ }_{S}{ }_{P}{ }_{H}$ C ${ }_{S}{ }_{P}{ }_{H}$ $\begin{array}{llll}C & S & P & H\end{array}$ C $S{ }_{P}{ }_{H}$ C ${ }_{S}{ }_{P}{ }_{H}$ C $S$ P ${ }^{3}$

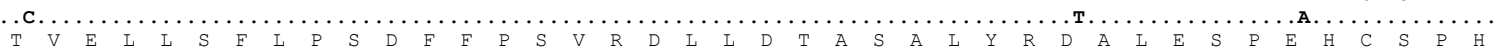

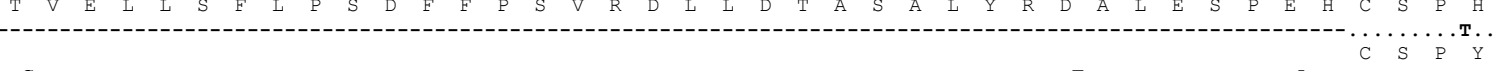

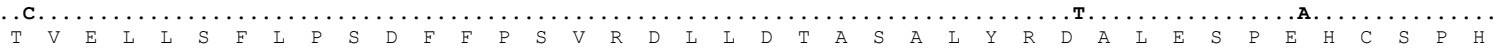

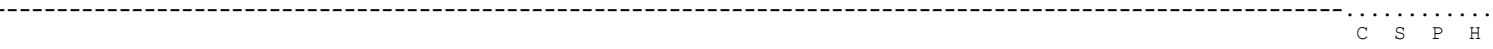

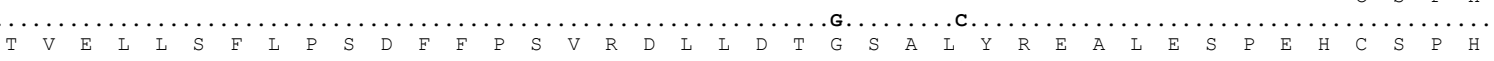

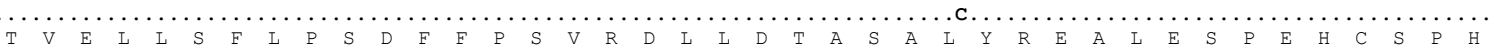

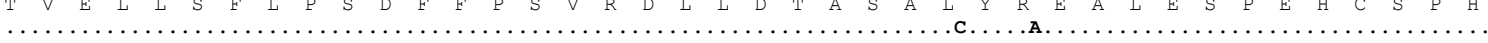

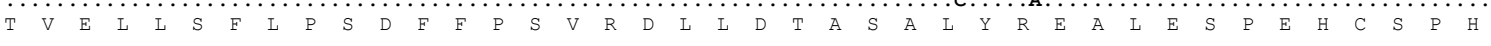
ACTGTGGAGTTACTCTCGTTTTTGCCTTCTGACTTCTTTCCTTCAGTAAGAGATCTTCTAGATACCGCCTCAGCTCTGTATCGGGATGCCTTAGAGTCTCCTGAGCATTGTTCACCTCAC $\begin{array}{llllllllllllllllllllllllllllllllllllllllll}T & V & E & L & L & S & F & L & P & S & D & F & F & P & S & V & R & D & L & L & D & T & A & S & A & L & Y & R & D & A & L & E & S & P & E & H & C & S & P & H\end{array}$

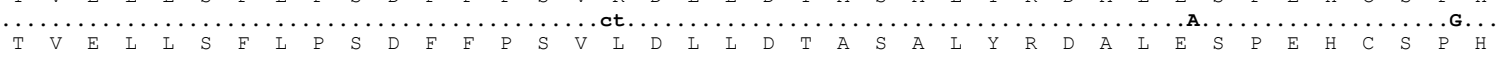


$\mathrm{X} 02763$

AH 2-4890

AH 1 . I

AH $\quad 4-1835$

AH 4-2015

AH 5-2878

AH 7-1884

AH 7-624

AH 7-640

AH $8-1993$

AH $10-2400$

AH 10-2596

AH 11-2641

AH $12-21$

AH $12-431$

CH 1-6126

CH 10-1982

CH 12-2805

CH 12-82

CH 5-2628

CH $6-444 \mathrm{~A}$

COMPL 4-2971

v01460

X02496

AH 10-1108

AH 10-1612

CH $10-3329$

AH $\quad$ 10-2085

AH $10-2425$

AH 10-737

AH $10-486$

AH 11-1941

AH 11-2336A

AH 11-2514

AH 11-2524

AH 11-2759

AH 11-2866

AH 11-5664

AH 11-965

AH $12-438$

AH 3-577

AH 2-2658

AH 3-7991

AH $11-8035$

AH 3-7755

AH $5-920$

AH 3-987

AH 4-2307

AH 4-3445

AH 4-775

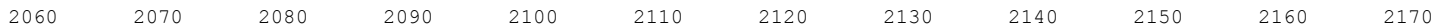
CATACTGCACTCAGGCAAGCATTCTCTGCTGGGGGAATTGATGACTCTAGCTACCTGGGTGGGTAATAATTTGGAAGATCCAGCATCTAGGGATCTTGTAGTAAATTATGTTAATACT

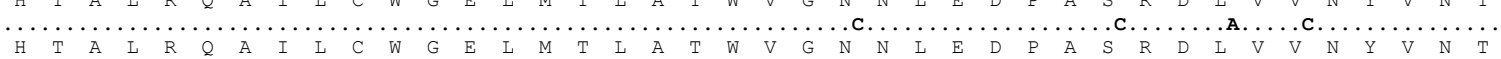

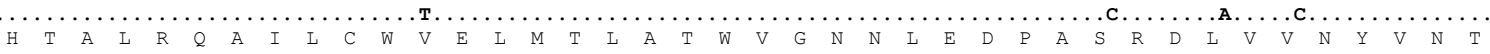

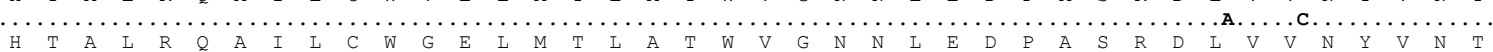

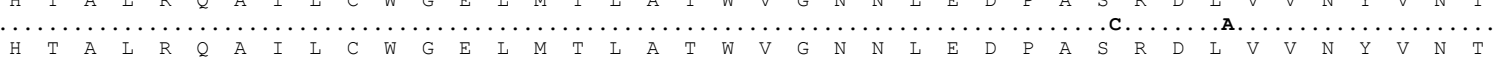

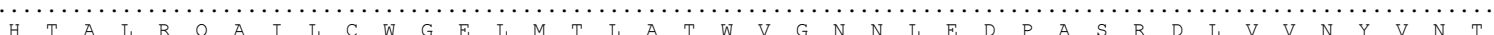

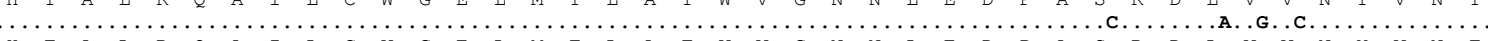

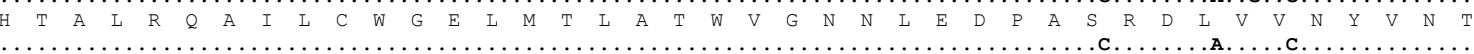

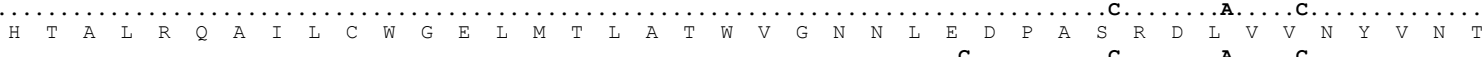

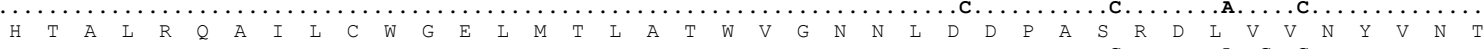

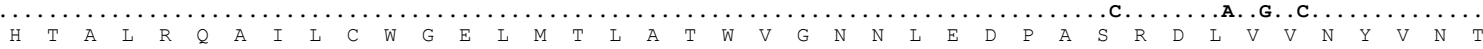

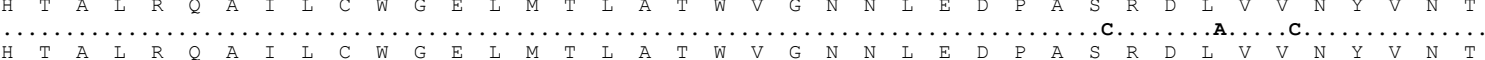

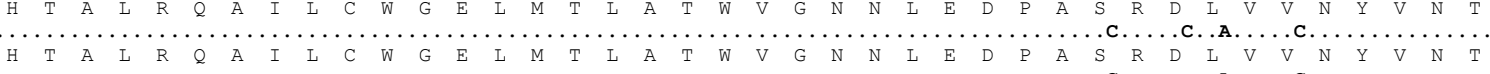

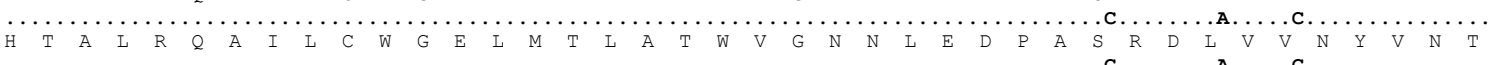

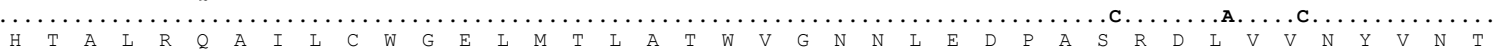

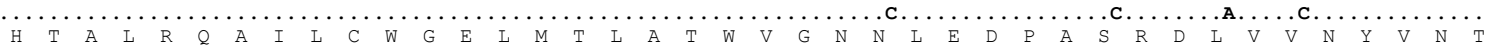

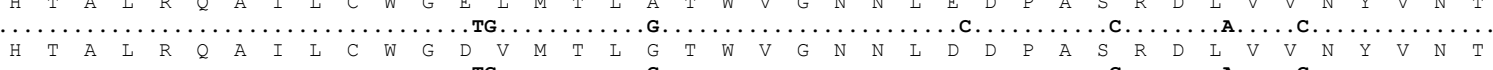

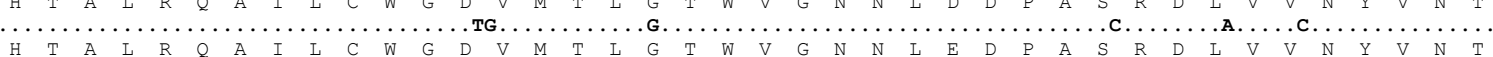

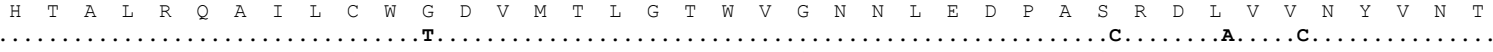

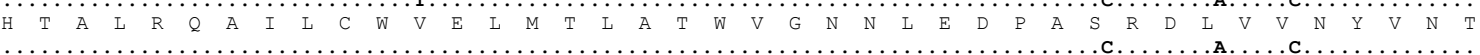
$H$
$H$
$H$

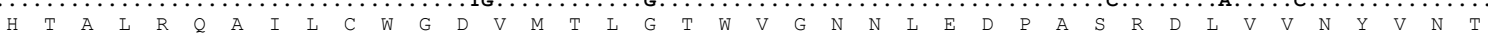

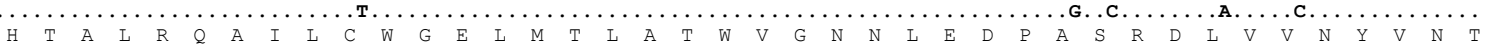

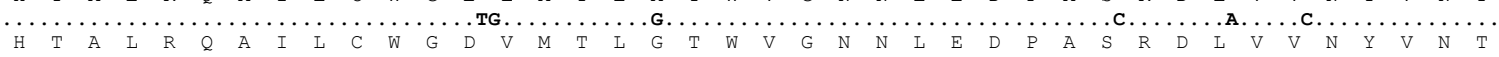

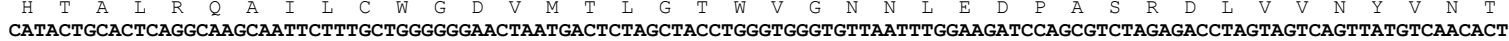

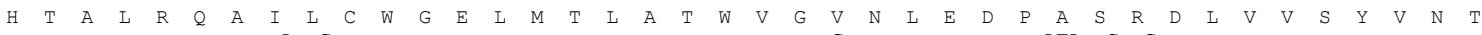

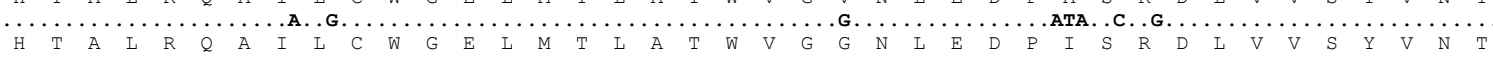

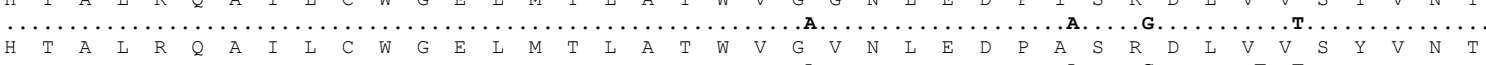

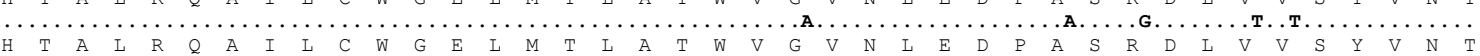

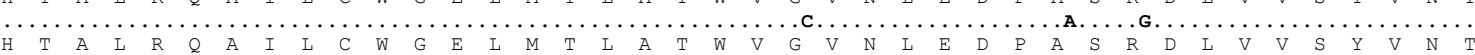

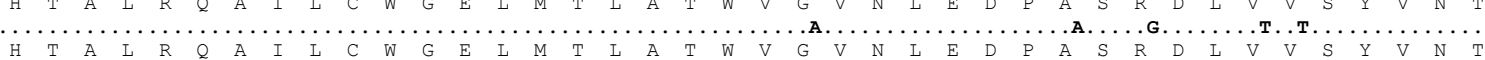

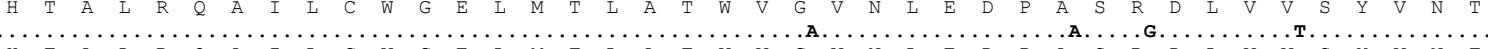

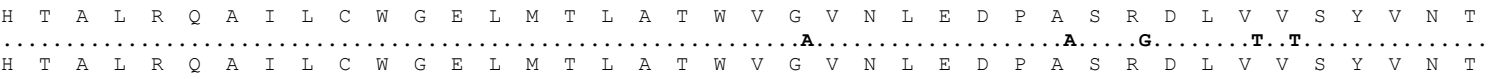

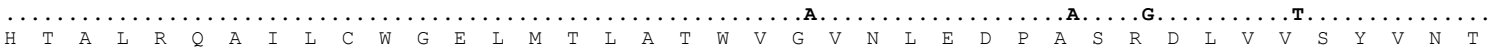

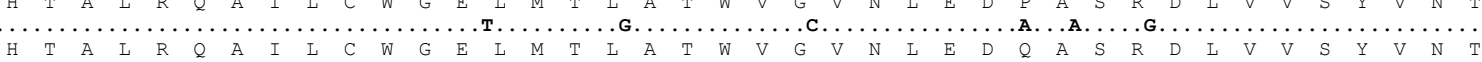

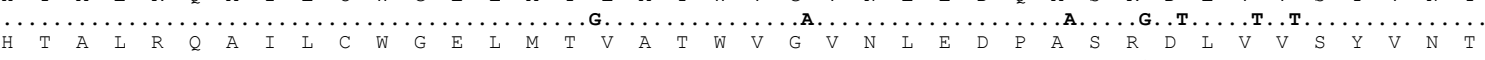

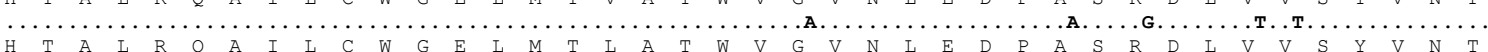

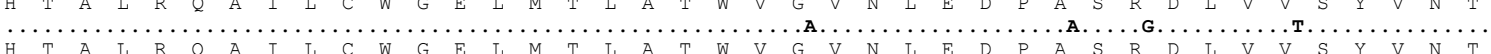

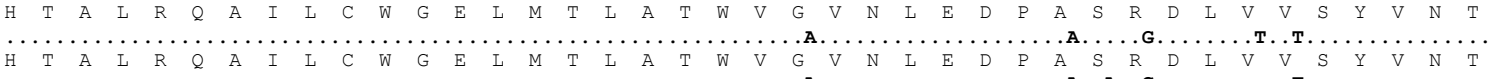

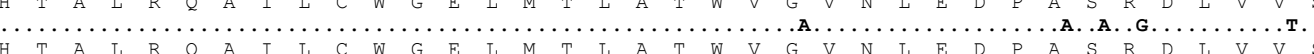

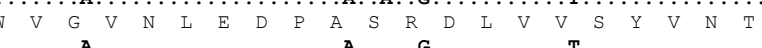

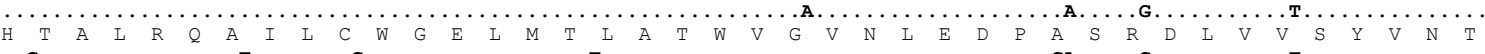

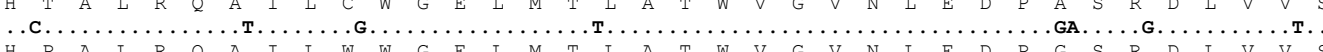

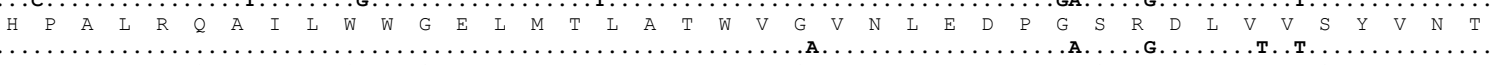

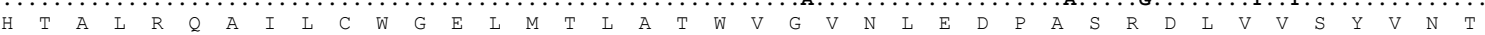

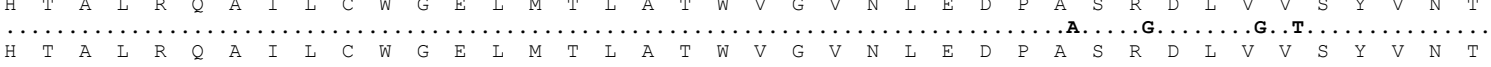

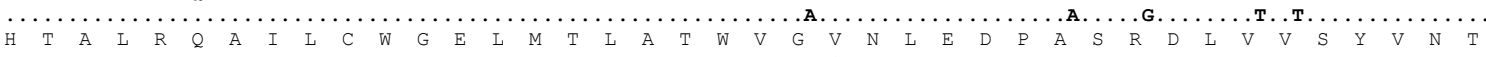

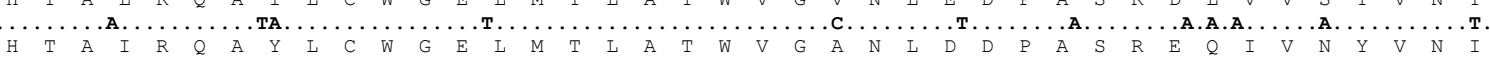

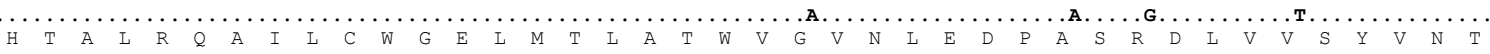

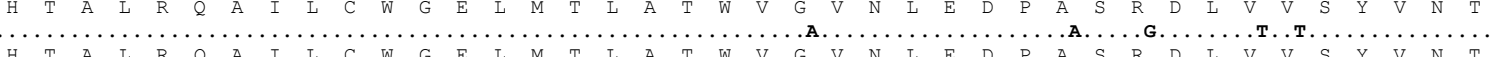

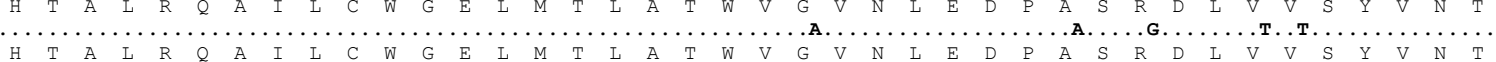

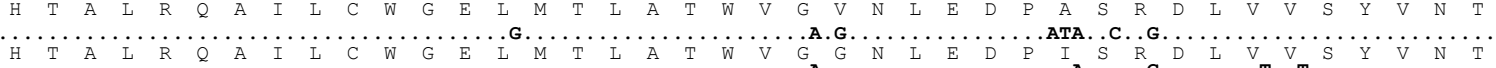

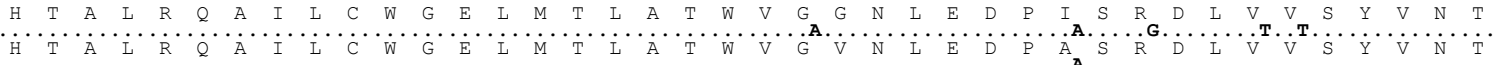

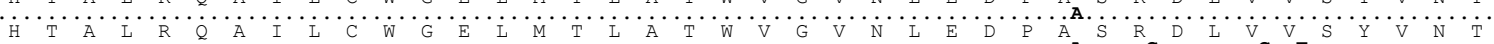

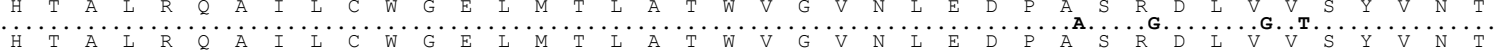


v01460

$\mathrm{x} 02496$

AH 4-778

AH 6.I

AH 5-556

AH 6-1289

AH 6-217

AH $6-220$

AH 6-2394

AH 6-2616

AH 7-1010

AH 7-1275

AH $7-1440$

AH 7-1442

AH 7-1501

AH 7-283

AH 7-3552

AH $8-1853$

AH $8-2186$

AH 9-2772

AH 9-296

AH 9-3113

AH 9-3163

AH 9-4070

CH $1-1546$

CH 1-4936

CH $\quad 10-3335$

CH 11-2343A

CH 11-2866

CH 11-5664

$\mathrm{CH}$ 11-884

CH 12-2178

CH 12-958

CH $6-2625$

CH 7-1117A

CH 7-2508

CH 9-599

CH 9-7181

COMPL 11-3028

COMPL 11-3179

COMPL 4-1725

COMPL 7-2083

X75657

AH 2-10101

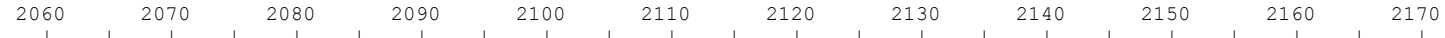

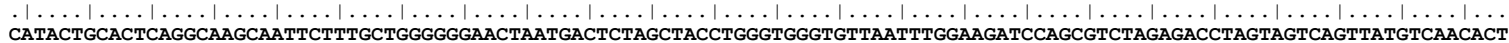
CATACTGCACTCAGGCAAGCAATTCTTTGCTGGGGGGACTAATGACTCTAGCTACCTGGGTGGGTGTTAATTTGGAAGATCCAGCGTCTAGAGACCTAGTAGTCAGTTATGTCAACACT

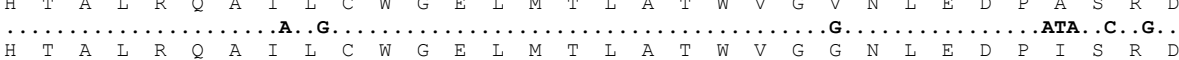

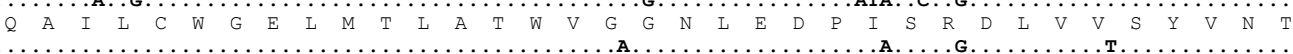

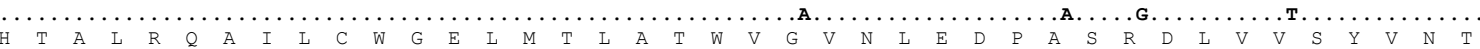

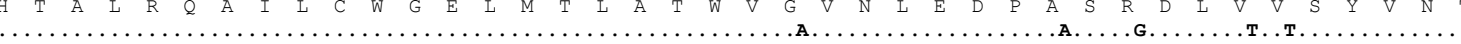

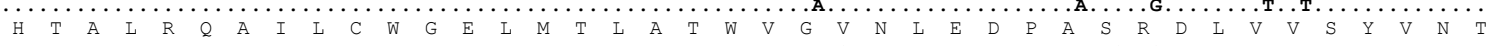

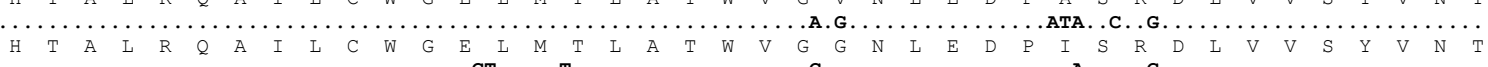

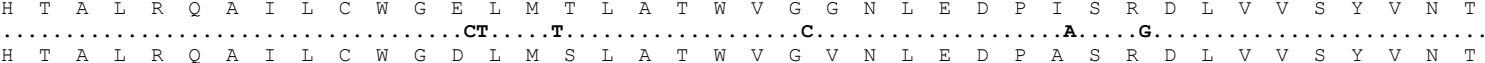

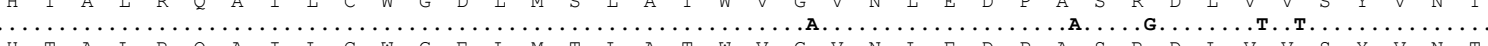

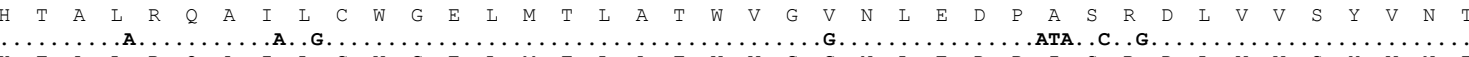

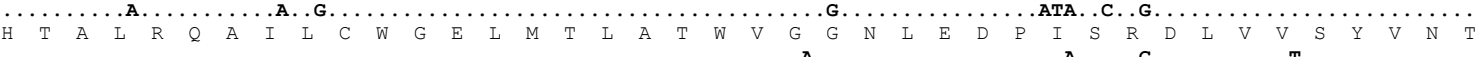

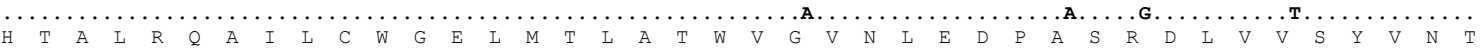

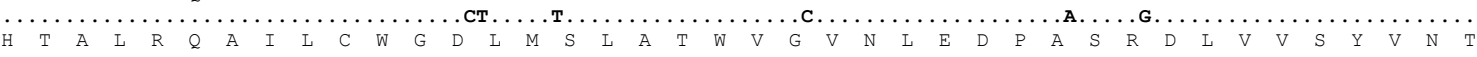

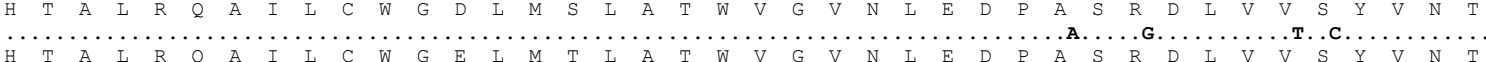

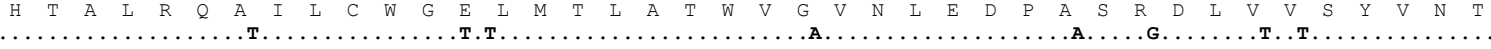

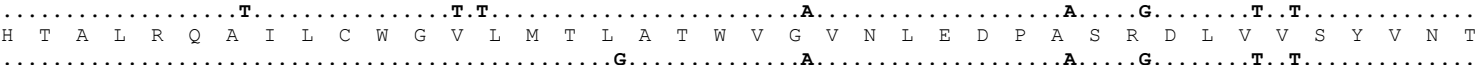

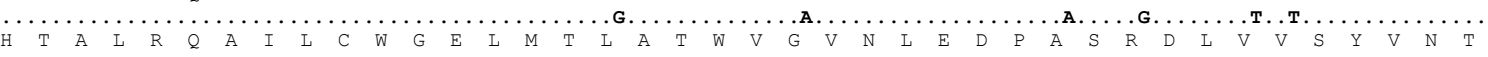

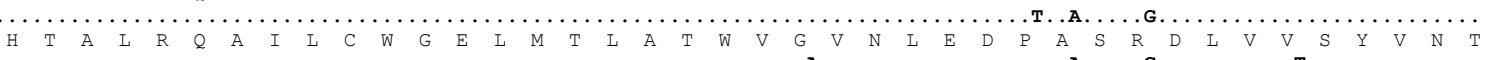

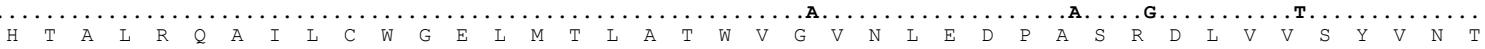

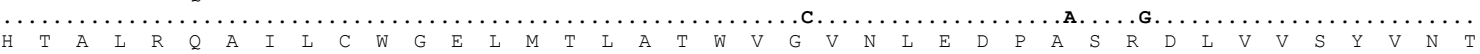

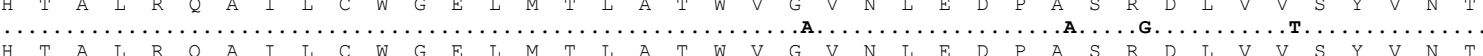

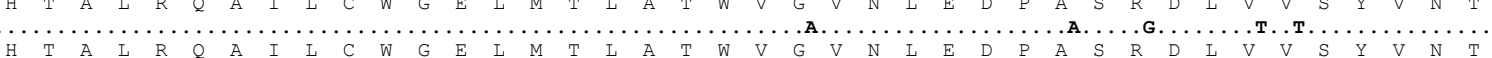

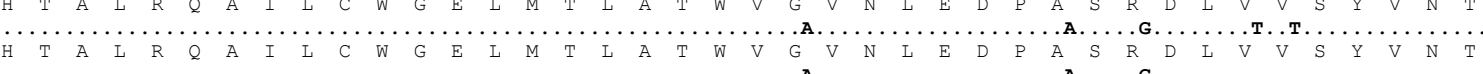

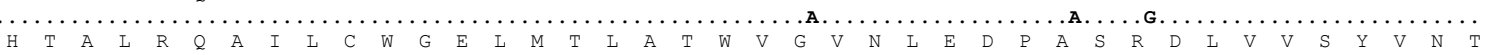

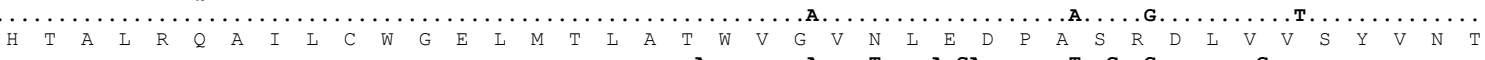

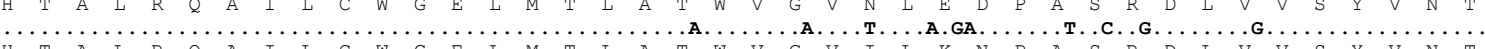

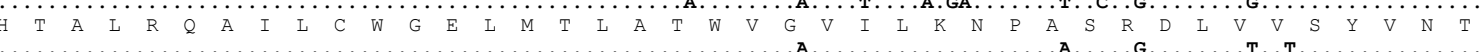

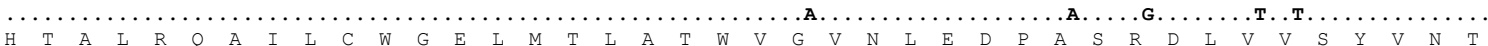

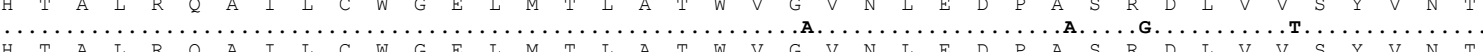

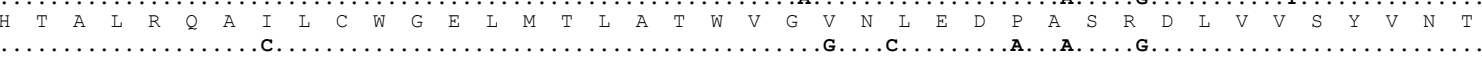

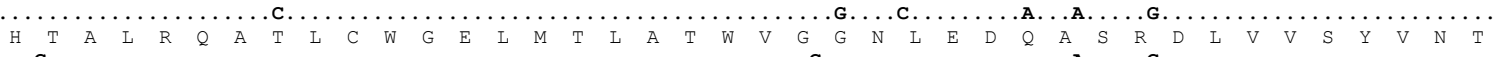

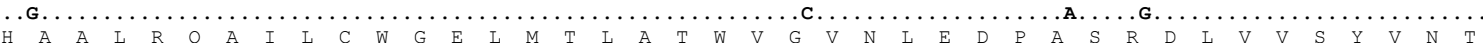

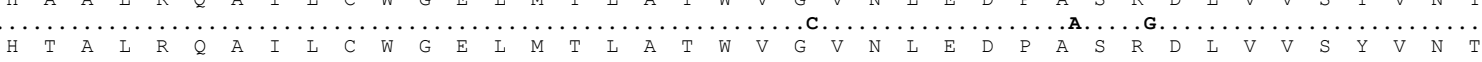

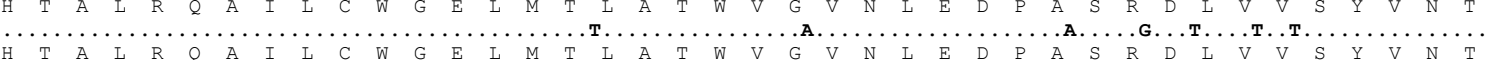

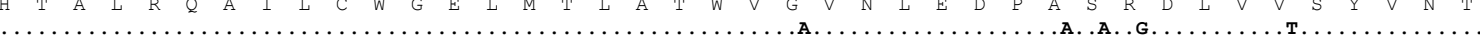

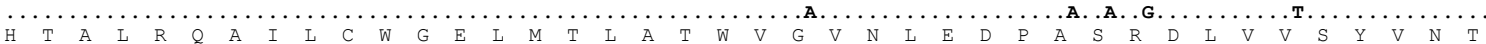

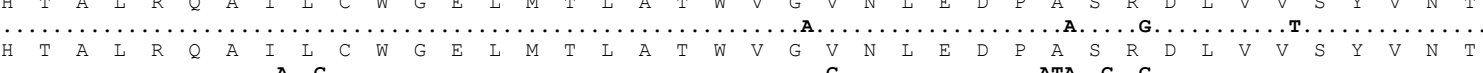

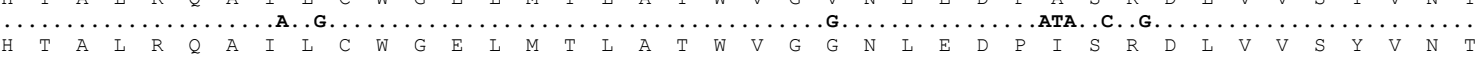

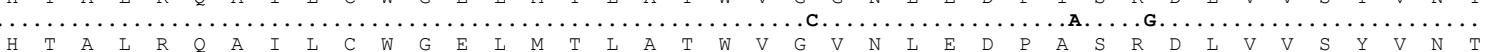

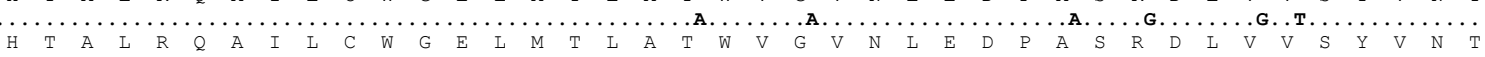

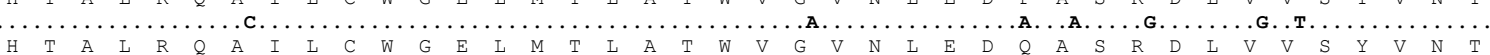

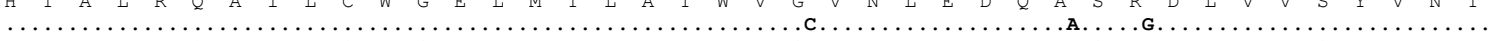

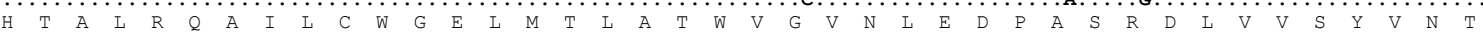

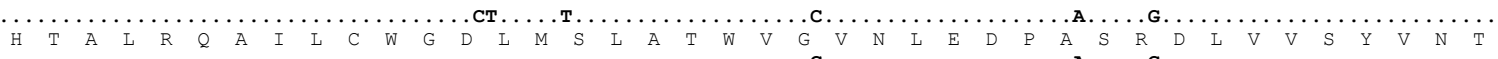

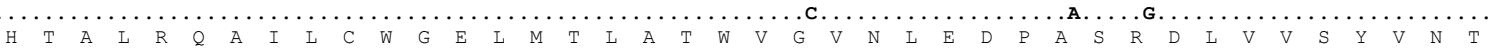

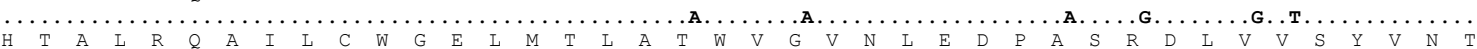

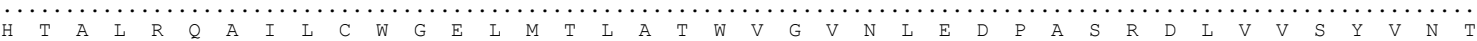
$H$
$H$

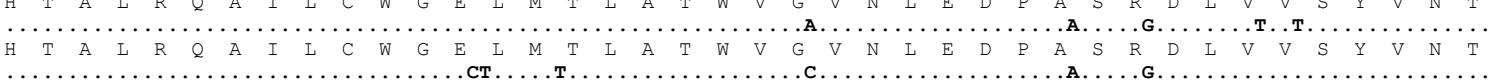

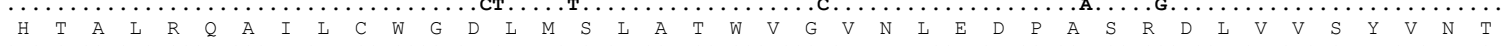
CACACTGCACTCAGGCAAGCCATTCTTTGCTGGGGAGAACTAATGACTCTAGCTACCTGGGTGGGTGTAAATTTGGAAGATCCAGCATCCAGGGACCTAGTAGTCAGTTATGTCAATACT

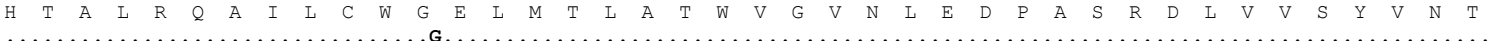

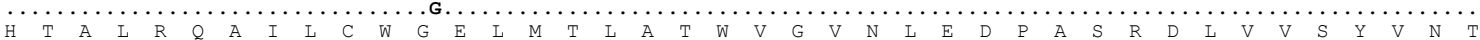


$\mathrm{X} 02763$

AH 2-4890

AH 1 . I

AH 4-1835

AH 4-2015

AH 5-2878

AH 7-1884

AH 7-624

AH 7-640

AH $8-1993$

AH $10-2400$

AH 10-2596

AH 11-2641

AH 12-21

AH $12-431$

CH 1-6126

CH 10-1982

CH $12-2805$

CH 12-82

CH 5-2628

CH 6-444A

COMPL 4-2971

v01460

X02496

AH $10-110$

AH $10-1612$

CH $10-3329$

AH $10-2085$

AH $\quad$ 10-2425

AH 10-737

AH $10-486$

AH $11-1941$

AH 11-2336A

AH 11-2514

AH 11-2524

AH 11-2759

AH 11-2866

AH 11-5664

AH 11-965

AH 12-438

AH 3-577

AH 2-2658

AH 3-7991

AH $11-8035$

AH 3-7755

AH $5-920$

AH 3-987

AH 4-2307

AH $4-3445$

AH 4-775

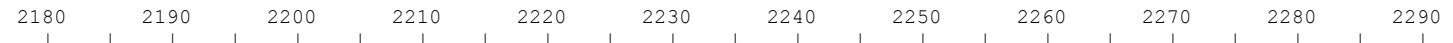

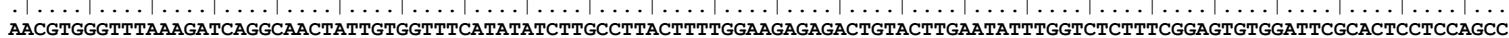
V G I I R Q I L $\begin{array}{lllllllllll}N & V & G & L & K & I & R & Q & L & I \\ N & M & G & L & K & I & R & Q & L & I\end{array}$

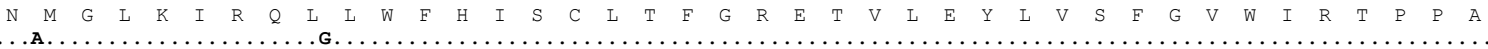

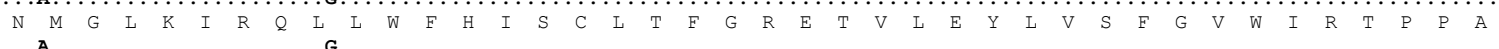

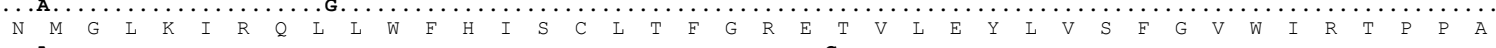

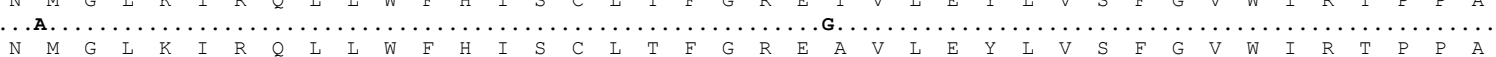

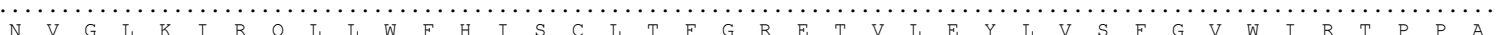

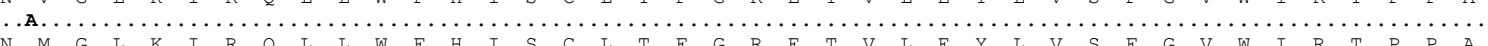
........

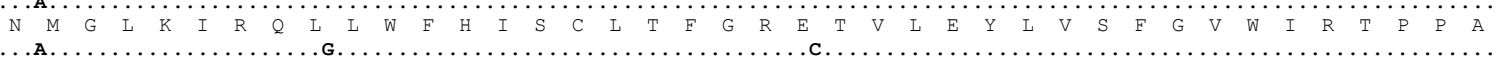

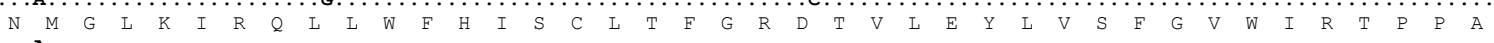

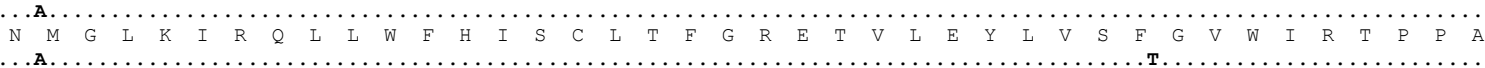

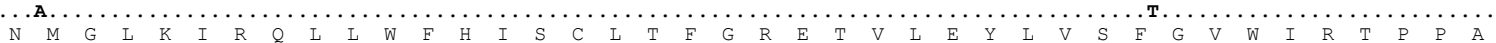

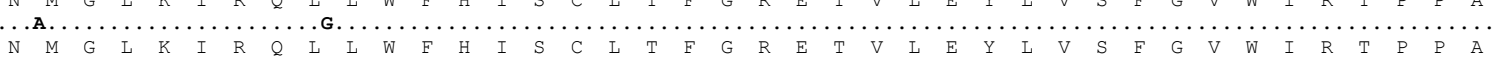

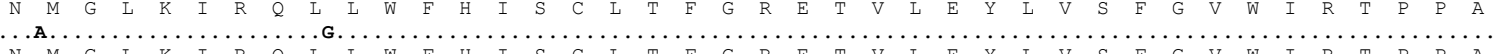

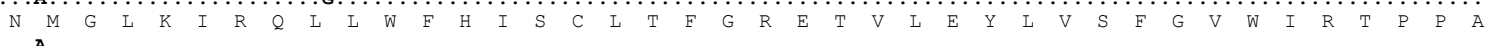

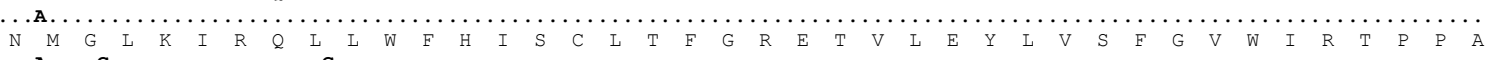

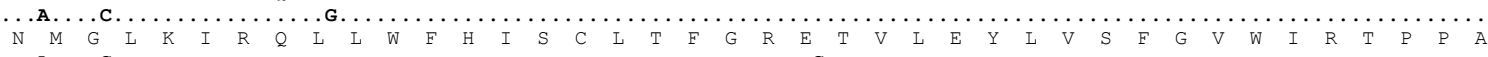

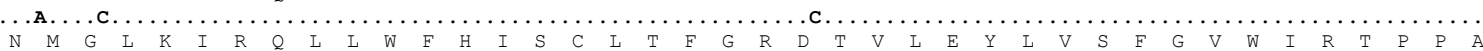

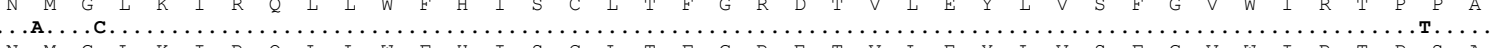

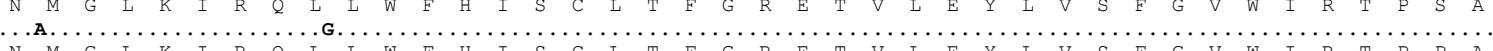

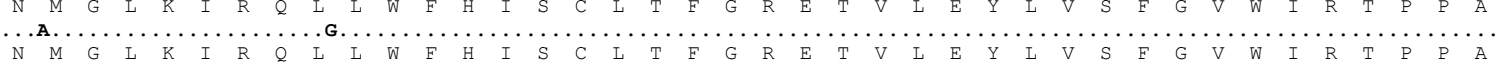

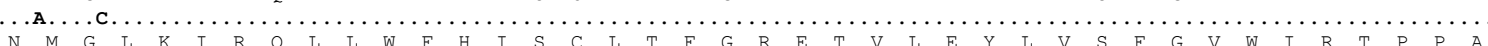

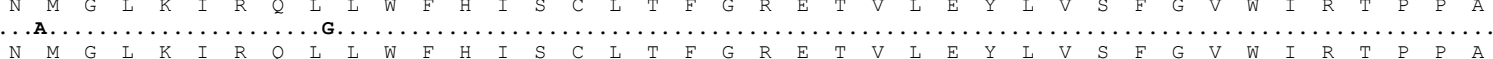
...............

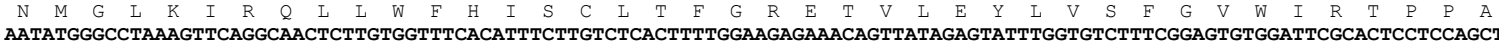
AATATGGGCCTAAAGTTCAGGCAACTCTTGTGGTTTCACATTTCTTGTCTCACTTTTGGAAGAGAAACAGTTATAGAGTATTTGGTGTCTTTCGGAGTGTGGATTCGCACTCCTCCAGCT

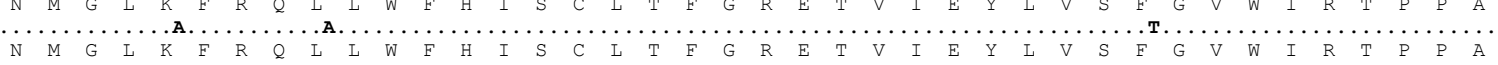

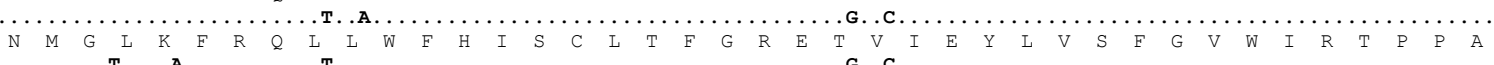

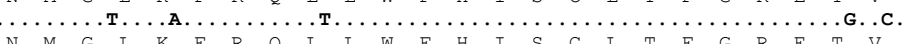

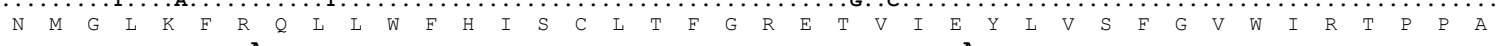

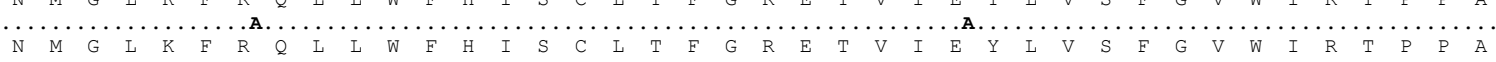

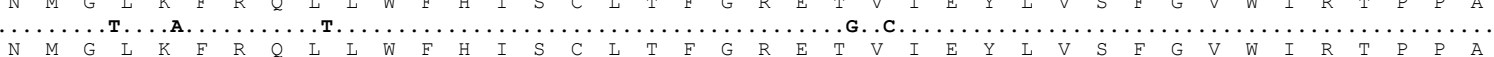

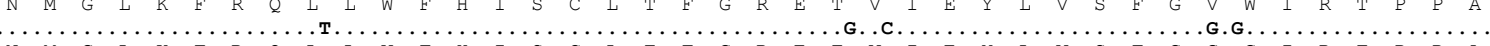

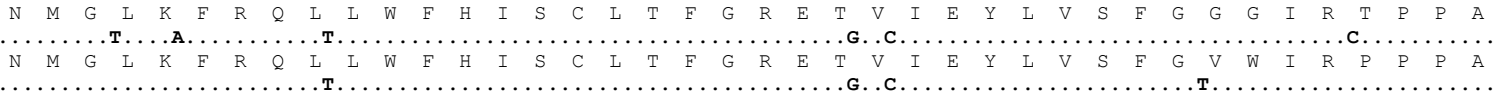

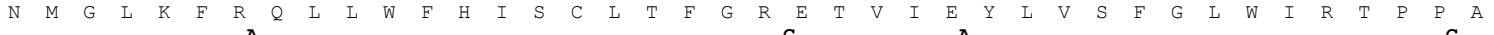

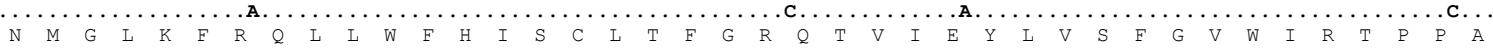

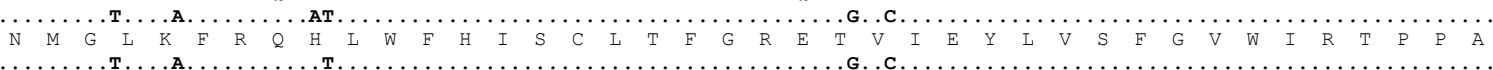

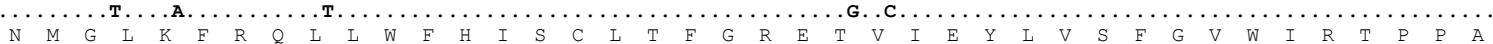

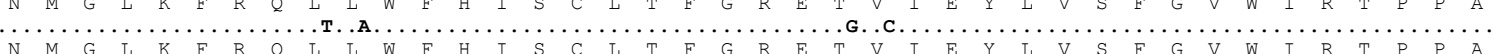

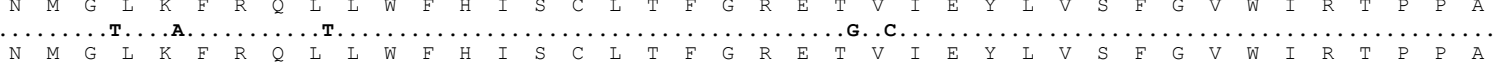

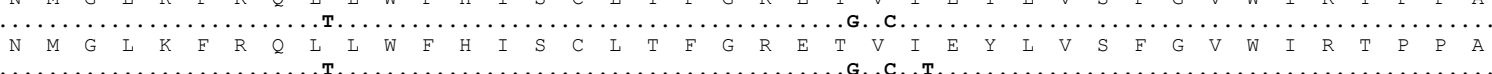

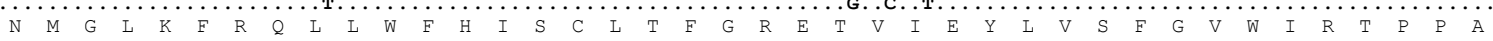
$W$
$N$ $\begin{array}{lllllllllllllllllllll}N & M & G & L\end{array}$

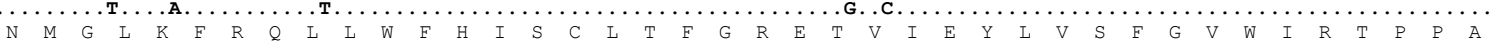

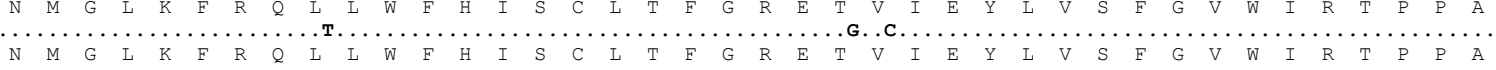

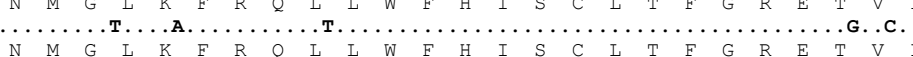

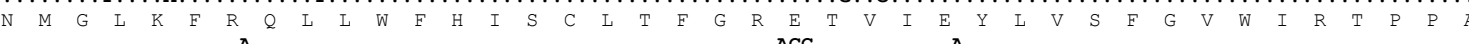

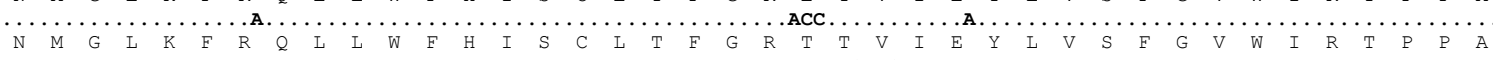

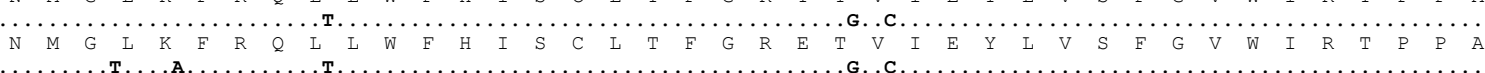

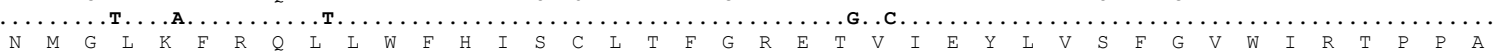

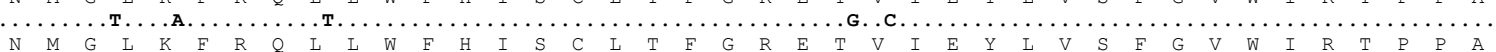

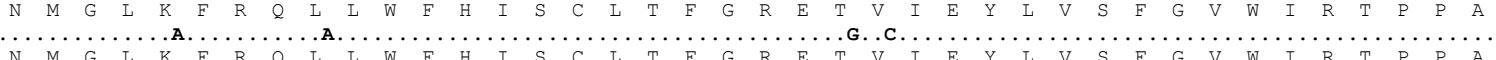

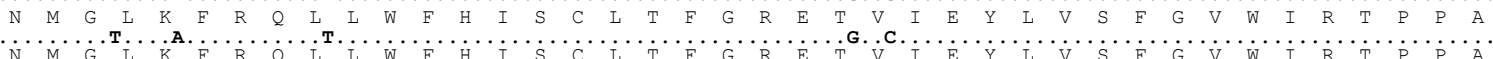

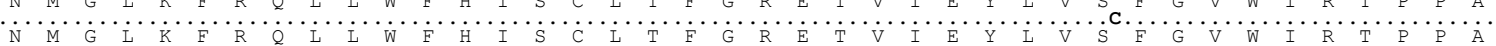

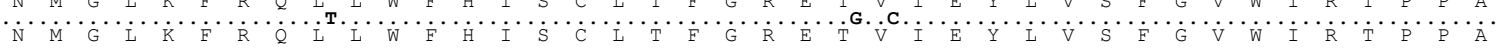


v01460

$\mathrm{x} 02496$

AH 4-778

AH 6. I

AH 5-556

AH 6-1289

AH 6-217

AH $6-220$

AH 6-2394

AH $6-2616$

AH 7-1010

AH 7-1275

AH 7-1440

AH 7-1442

AH 7-1501

AH 7-283

AH 7-3552

AH $8-1853$

AH $8-2186$

AH 9-2772

AH 9-296

AH 9-3113

AH 9-3163

AH 9-4070

CH $1-1546$

CH 1-4936

CH $\quad 10-3335$

CH 11-2343A

CH 11-2866

CH 11-5664

$\mathrm{CH}$ 11-884

CH $\quad 12-2178$

CH 12-958

CH $\quad 6-2625$

CH 7-1117A

CH 7-2508

CH 9-599

CH 9-7181

COMPL 11-3028

COMPL 11-3179 COMPL 4-1725

COMPL 7-2083

$\times 75657$

AH 2-10101

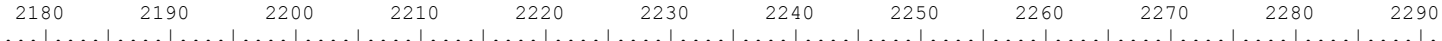

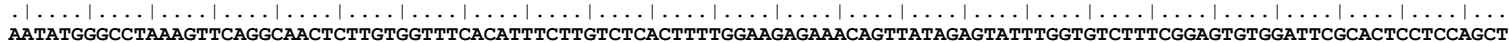
AATATGGGCCTAAAGTTCAGGCAACTCTTGTGGTTTCACATTTCTTGTCTCACTTTTGGAAGAGAAACAGTTATAGAGTATTTGGTGTCTTTCGGAGTGTGGATTCGCACTCCTCCAGCT

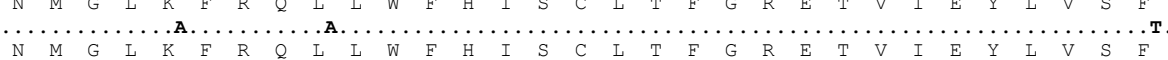

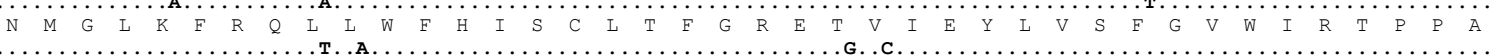

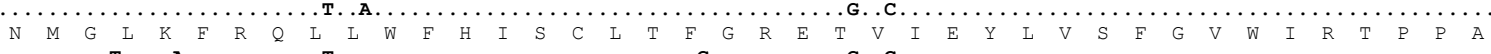

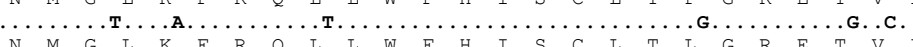

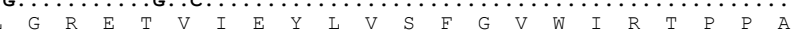
$\underset{N}{M} \begin{aligned} & M \\ & M\end{aligned}$

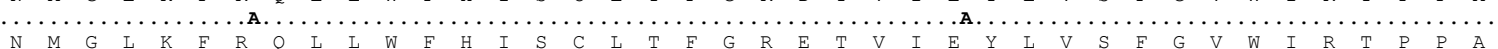

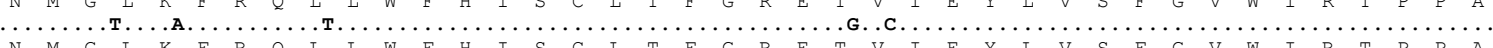

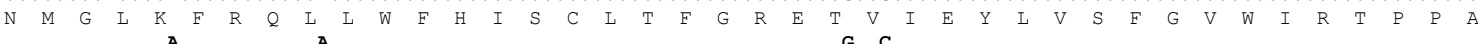

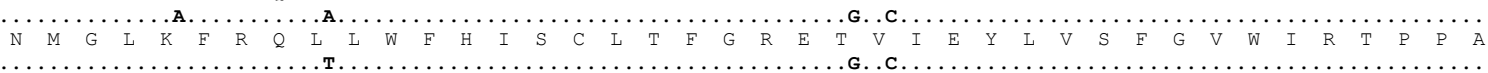

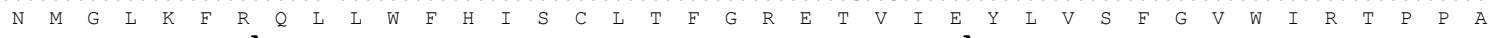

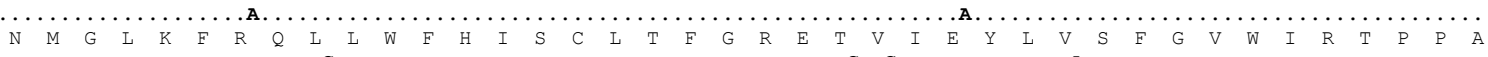

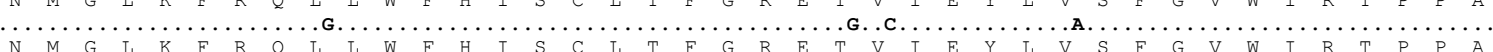

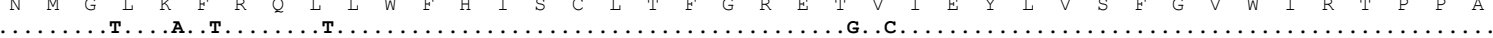

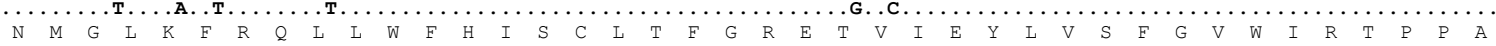
$N$
$N$

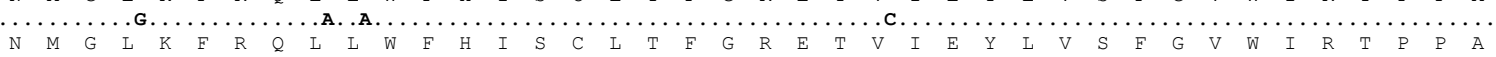

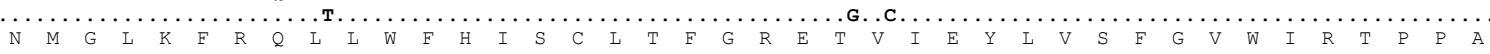

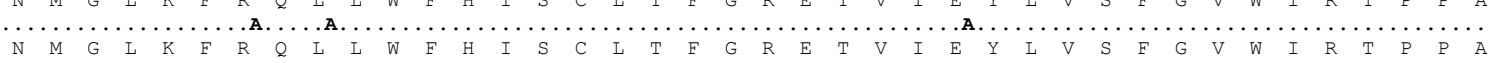

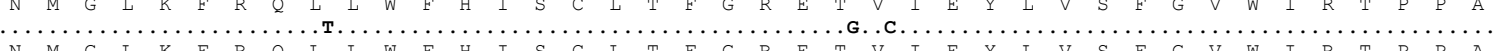

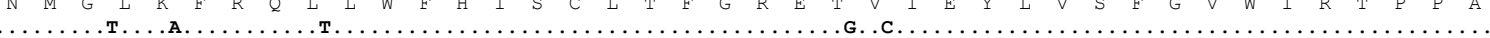
$\underset{N}{M} \begin{aligned} & M \\ & M\end{aligned}$

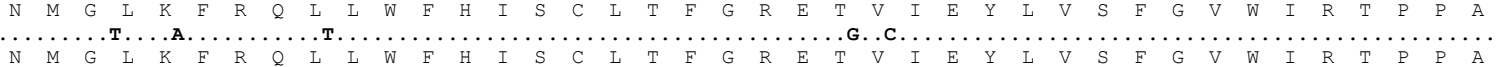

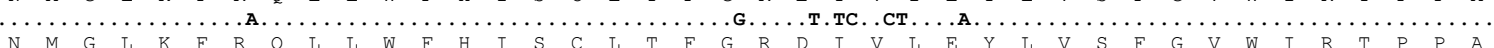

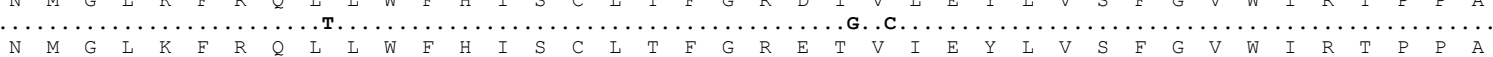

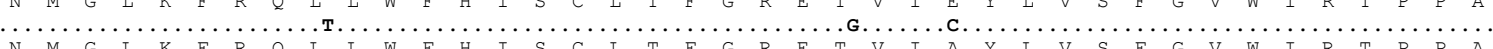

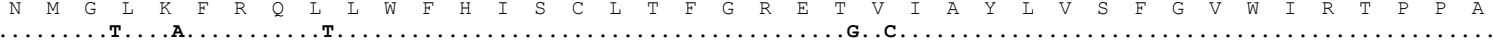

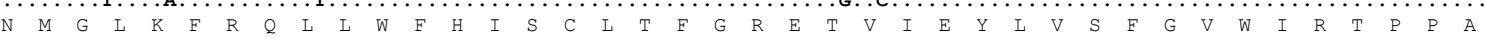

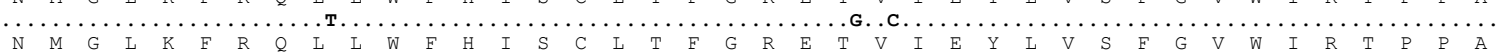

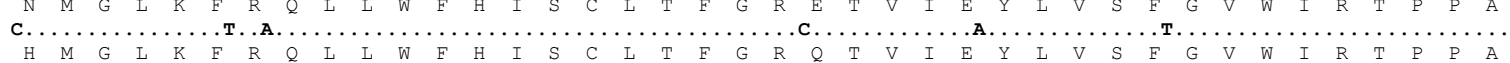

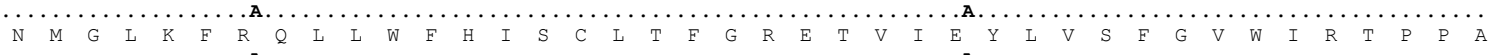

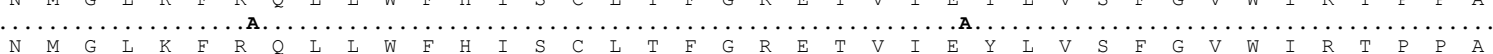

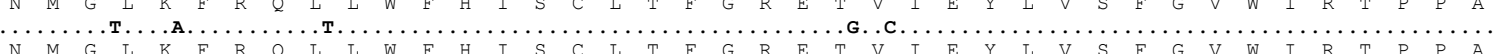

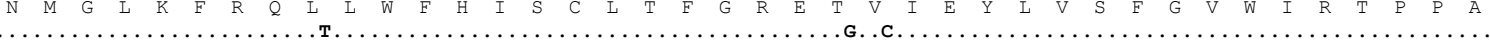

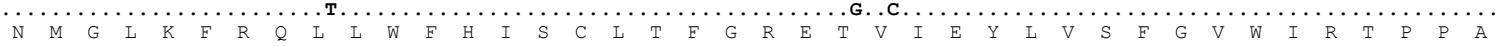

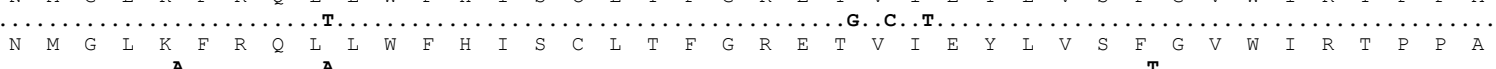

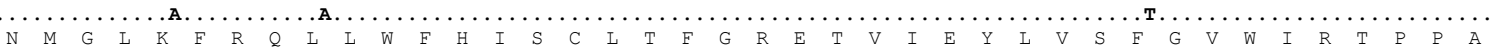

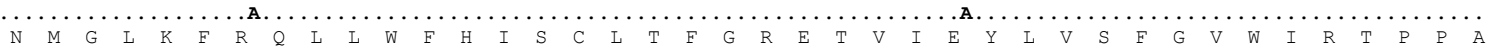

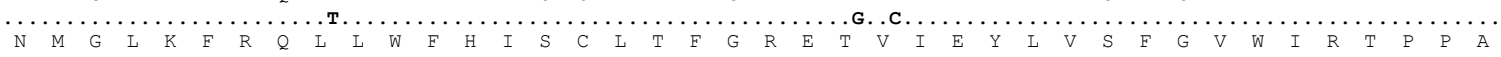

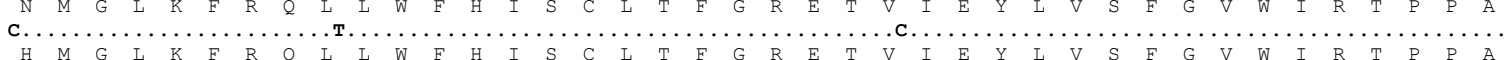

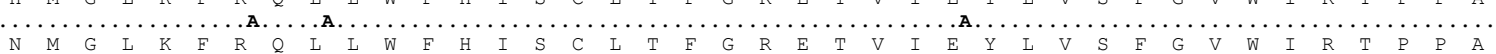

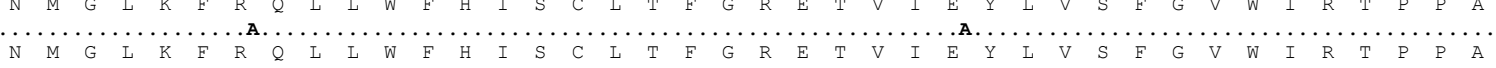

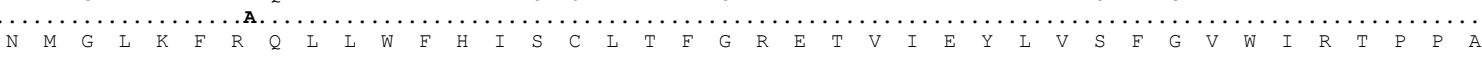

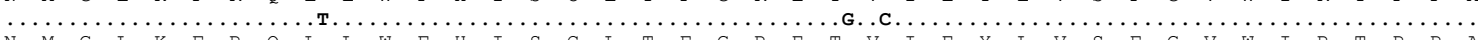

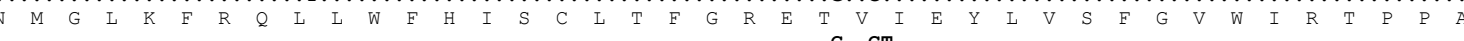

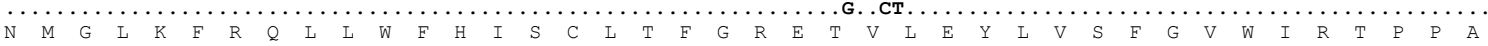

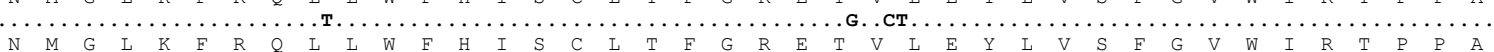

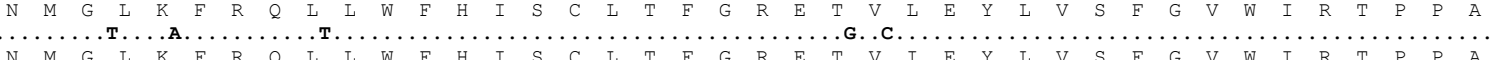

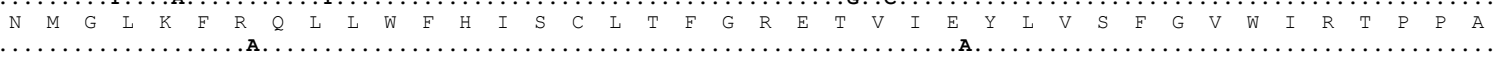

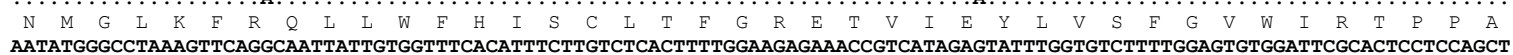
A M G T MAATTCAGGCAATTATTGTGGTTTCACATTTCTTGTCTCACTTTTGGAAGAGAAACCGTCATAGAGTATTTGGTGTCTTTTGGAGTGTGGATTCGCACTCCTCCAGCT

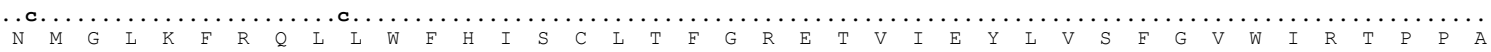


$\mathrm{X} 02763$

AH 2-4890

AH 1 . I

AH 4-1835

AH 4-2015

AH 5-2878

AH 7-1884

AH 7-624

AH 7-640

AH $8-1993$

AH $10-2400$

AH 10-2596

AH 11-2641

AH $12-21$

AH $12-431$

CH $1-6126$

CH 10-1982

CH $12-2805$

CH 12-82

CH 5-2628

CH $6-444 \mathrm{~A}$

COMPL 4-2971

v01460

X02496

AH 10-1108

AH $10-1612$

CH $10-3329$

AH $\quad 10-2085$

AH $\quad$ 10-2425

AH 10-737

AH 10-486

AH 11-1941

AH 11-2336A

AH 11-2514

AH 11-2524

AH 11-2759

AH 11-2866

AH 11-5664

AH 11-965

AH 12-438

AH 3-577

AH 2-2658

AH 3-7991

AH $11-8035$

AH 3-7755

AH $5-920$

AH 3-987

AH 4-2307

AH $4-3445$

AH $4-775$

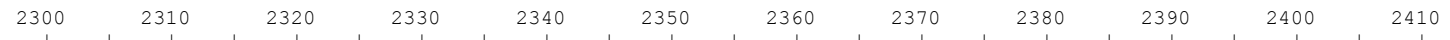
TATAGACCACCAAATGCCCCTATCTTATCAACACTTCCGGAACTACTGTTGTTAGACGACGGGACCGAGGCAGGTCCCCTAGAAGAAGAACTCCCTCGCCTCGCAGACGCAGATCTCCA $Y \quad R \quad \ldots$ $Y$
$Y$

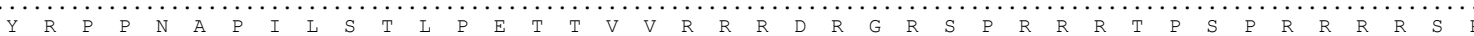

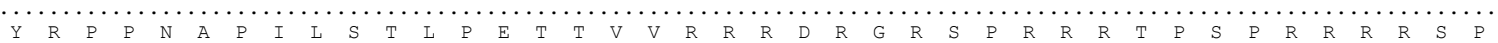

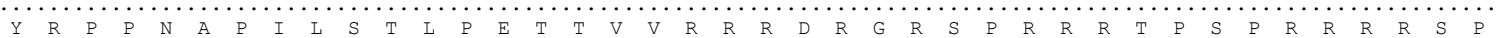

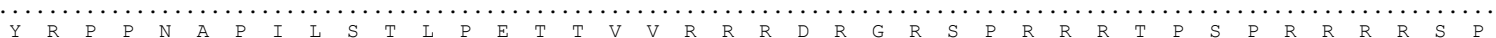

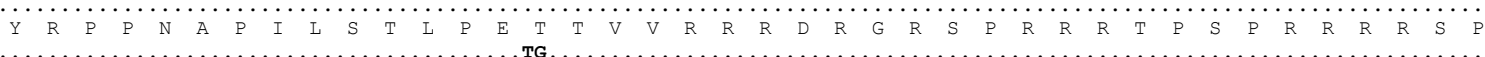

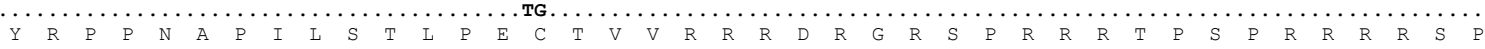

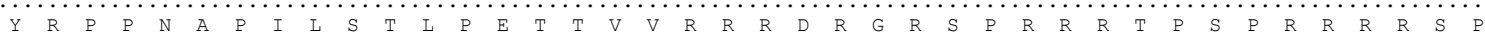

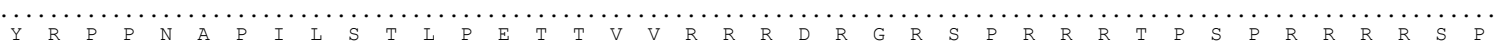

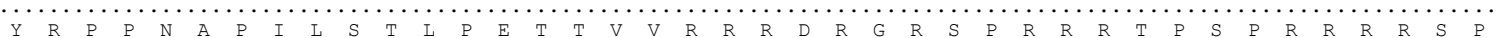

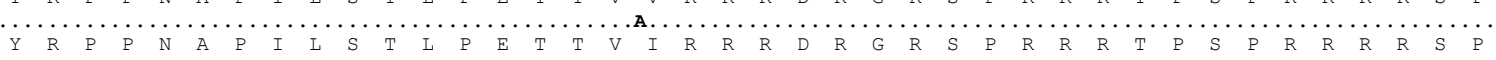

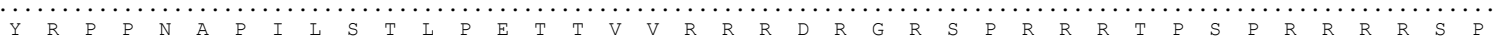

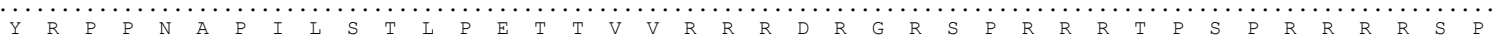

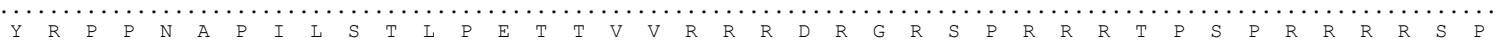

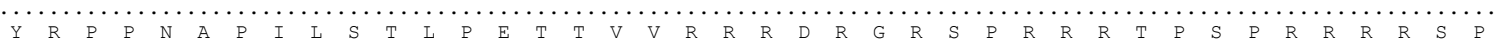

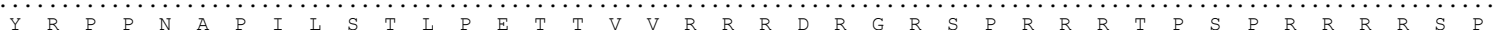

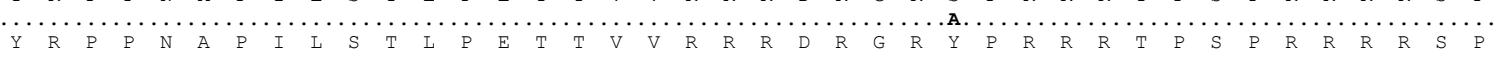

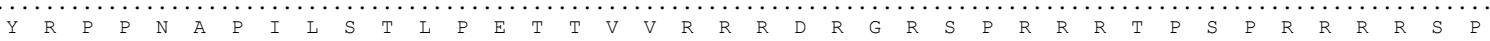

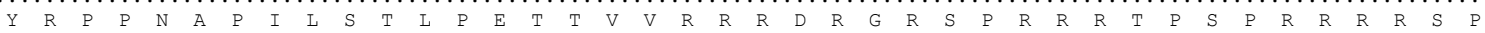
$Y$
$P$ TATAGACCACCAAATGCCCCTATCCTATCAACACTTCCGGAGACTACTGTTGTTAGACGACG------AGGCAGGTCCCCTAGAAGAAGAACTCCCTCGCCTCGCAGACGAAGGTCTCAA

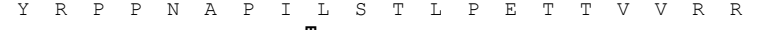

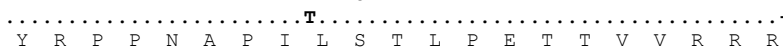

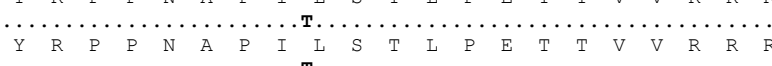

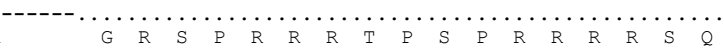

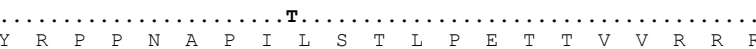

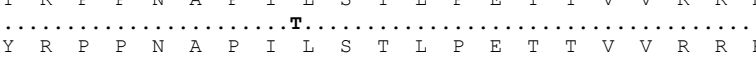

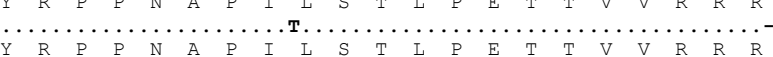

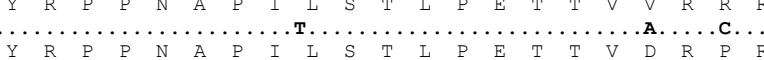

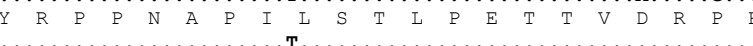

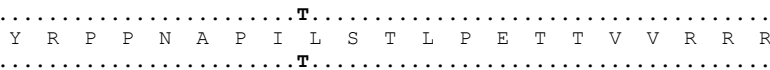
$\begin{array}{llllllllllllllllllllllll}Y & R & P & P & N & A & P & I & L & S & T & L & P & E & T & T & V & V & R & R & R\end{array}$

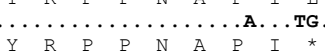
$\ldots \ldots \ldots \ldots \ldots \ldots \ldots \ldots \ldots$ T.

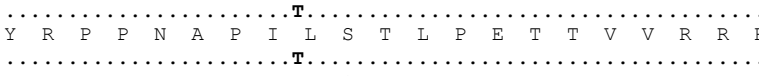
$\begin{array}{lllllllllllllllllll} & R & P & P & N & A & P & I & L & S & T & L & P & E & T & T & V & V & R \\ R\end{array}$

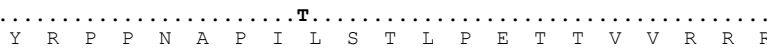

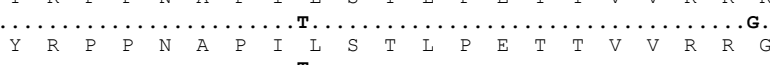

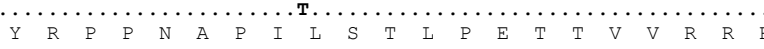

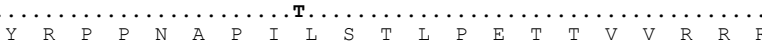

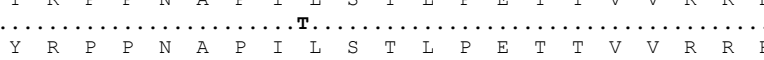

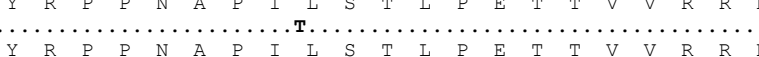

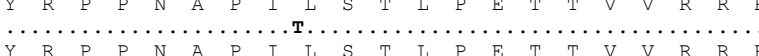

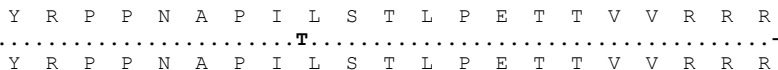

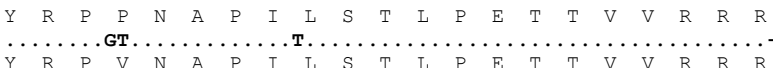

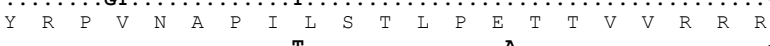

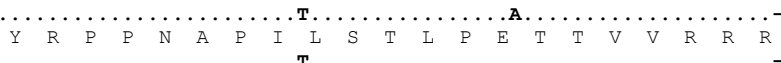

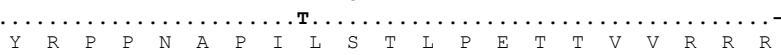

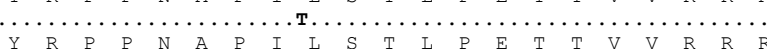

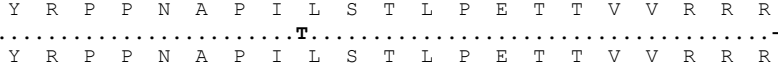

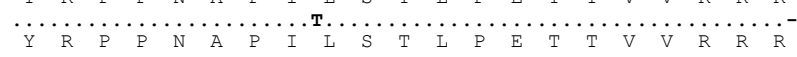

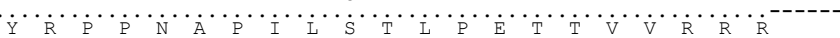

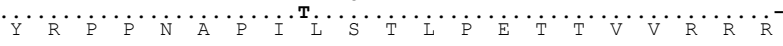

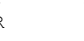
${ }_{G}{ }_{R}{ }_{S}{ }_{P}{ }_{R}{ }_{R}{ }_{R}{ }_{T}{ }_{P}{ }_{S}{ }_{P}{ }_{R}{ }_{R}{ }_{R}{ }_{R}{ }_{S}{ }_{Q}$

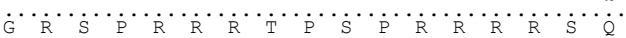


v01460

$\mathrm{x} 02496$

AH $4-778$

AH $6 . I$

AH 5-556

AH 6-1289

AH 6-217

AH $6-220$

AH 6-2394

AH 6-2616

AH 7-1010

AH 7-1275

AH 7-1440

AH 7-1442

AH 7-1501

AH 7-283

AH 7-3552

AH $8-1853$

AH $8-2186$

AH 9-2772

AH 9-296

AH $9-3113$

AH 9-3163

AH 9-4070

CH $1-1546$

CH 1-4936

CH $10-3335$

CH 11-2343A

CH 11-2866

CH 11-5664

$\mathrm{CH}$ 11-884

CH $\quad 12-2178$

CH 12-958

CH 6-2625

CH 7-1117A

CH 7-2508

CH 9-599

CH 9-7181

COMPL 11-3028

COMPL 11-3179

COMPL 4-1725

COMPL 7-2083

X75657

AH 2-10101

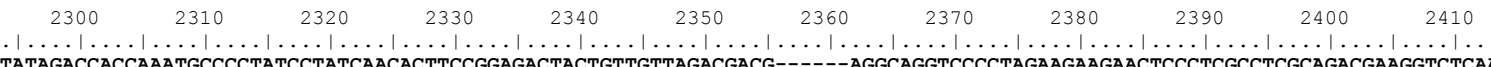
TATAGACCACCAAATGCCCTATCCTATCAACACTTCCGGAGACTACTGTTGTTAGACGACG----AGGCAGGTCCCTAGAAGAAGAACTCCTCGCCTCGCAGACGAGGTCTCAA

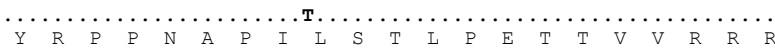

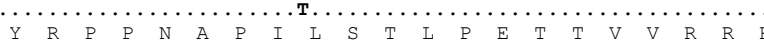

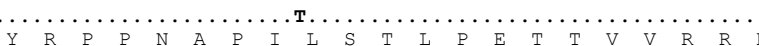

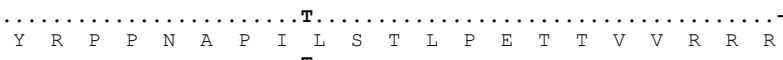
$Y$
$Y$

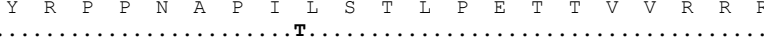

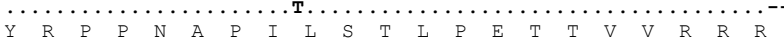

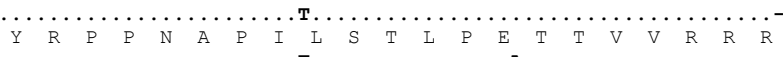

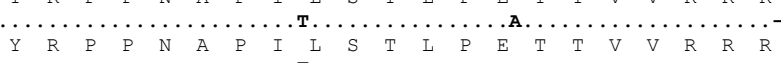

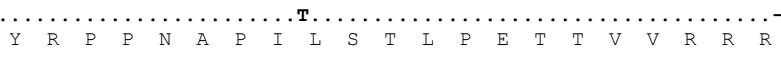

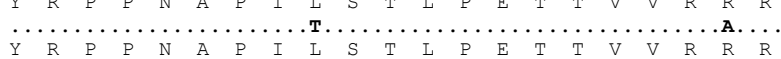

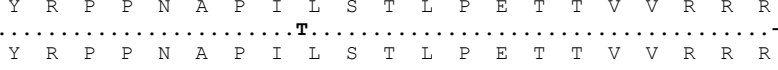

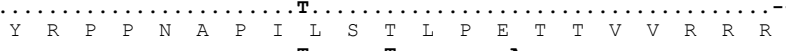

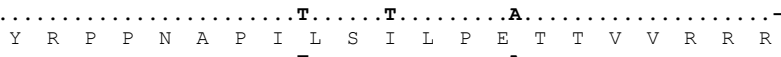

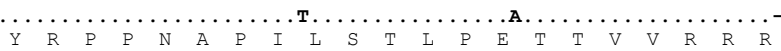

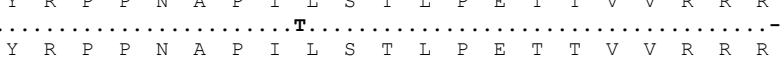

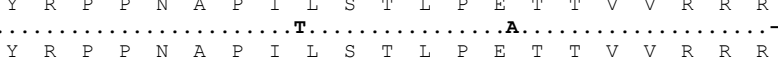

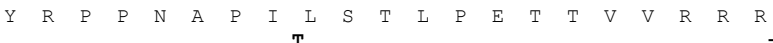

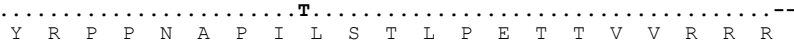

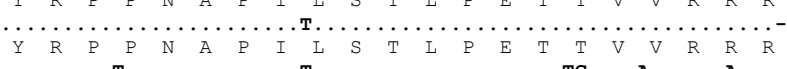

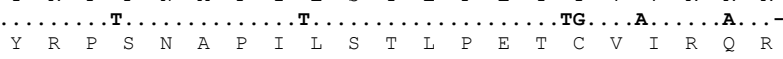

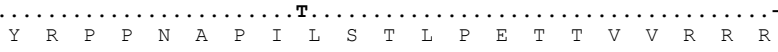

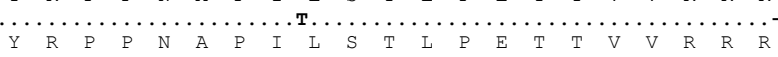

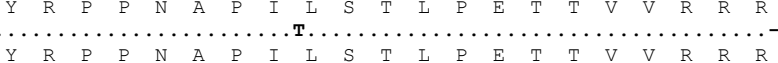

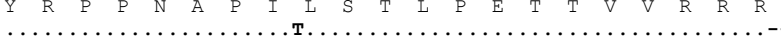

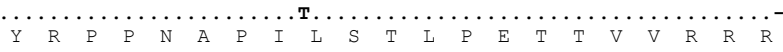

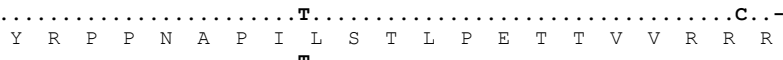

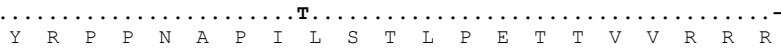

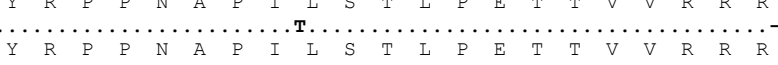

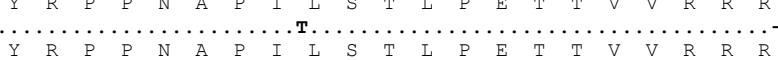

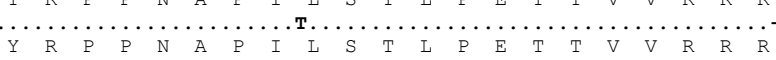

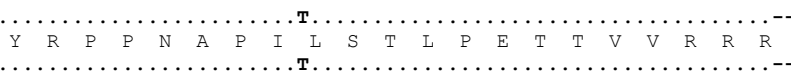
$\begin{array}{lllllllllllllllllllll}Y & R & P & P & N & A & P & I & L & S & T & L & P & E & T & T & V & V & R & R\end{array}$

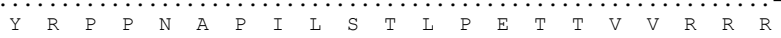

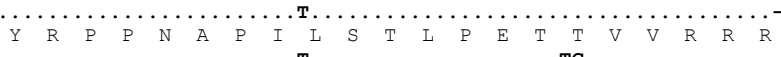

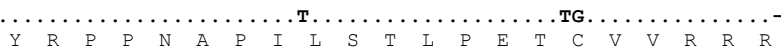

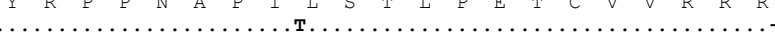

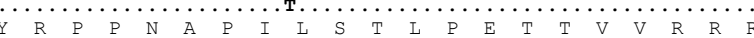

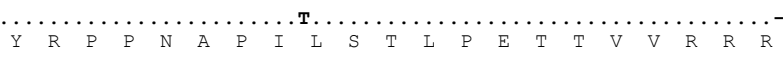

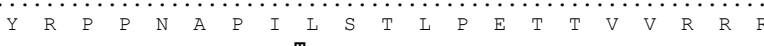

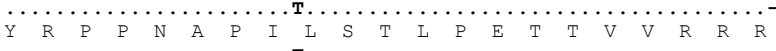

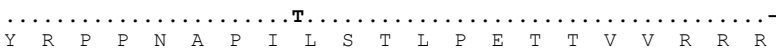

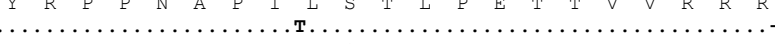

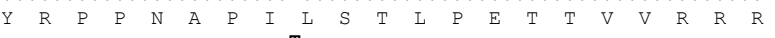

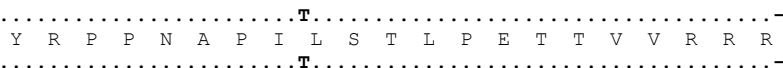

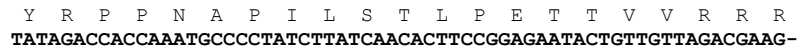
TATAGACCACCAAATGCCCCTATCTTATCAACACTTCCGGAGAATACTGTTGTTAGACGAAG-

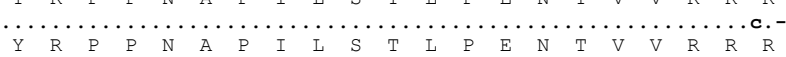

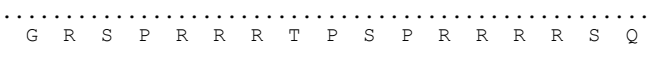

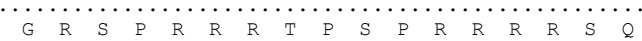

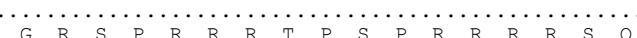

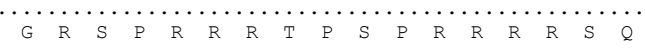

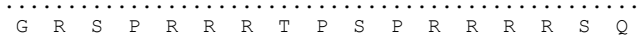

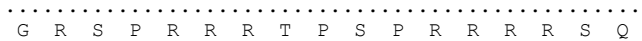
$\begin{array}{lllllllllllllllll}G & R & S & P & R & R & R & T & P & S & P & R & R & R & R & S & Q\end{array}$

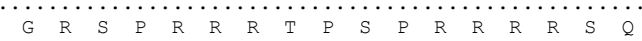

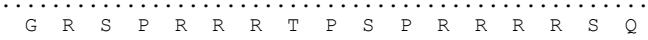

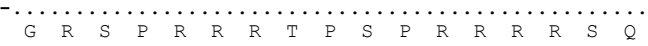

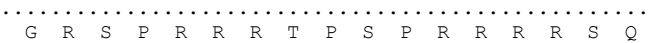
$\begin{array}{llllllllllllllllllllllll}G & R & S & P & R & R & R & T & P & S & P & R & R & R & R & S & Q\end{array}$

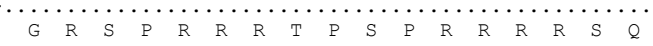

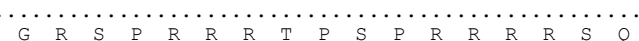

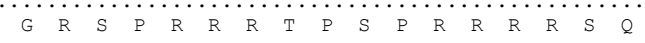

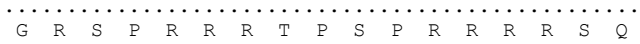

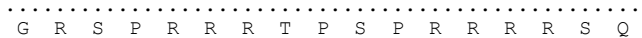

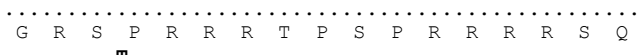

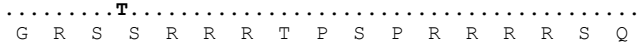

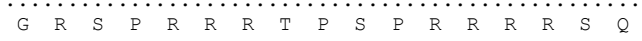

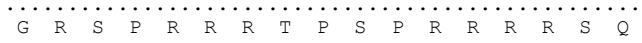

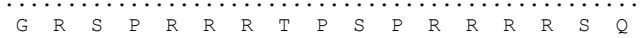

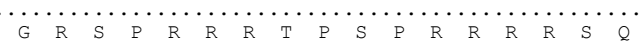

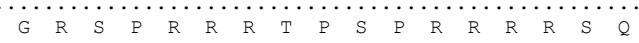

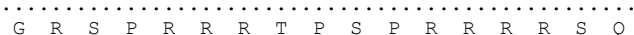

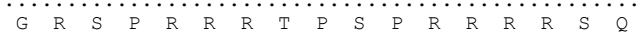
$\begin{array}{llllllllllllllll}G & R & S & P & R & R & R & T & P & S & P & R & R & R & R & S\end{array}$

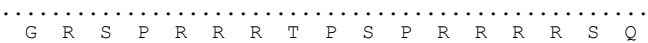
$\begin{array}{lllllllllllllllll}G & R & S & P & R & R & R & T & P & S & P & R & R & R & R & S & Q\end{array}$

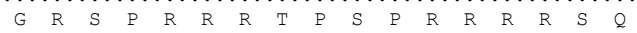

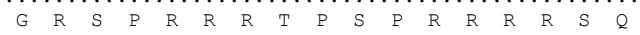

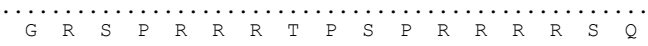

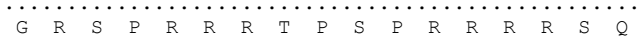
${ }_{G}{ }_{R}{ }_{S}{ }_{P}{ }_{R}{ }_{R}{ }_{R}{ }_{T}{ }_{P}{ }_{S}{ }_{P}{ }_{R}{ }_{R}{ }_{R}{ }_{R}{ }_{S}{ }_{Q}$

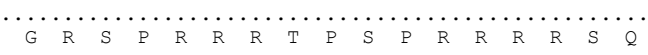
$\begin{array}{lllllllllllllllll}G & R & S & P & R & R & R & T & P & S & P & R & R & R & R & S & Q\end{array}$ $\begin{array}{llllllllllllllll}G & R & S & P & R & R & R & T & P & S & P & R & R & R & R & S\end{array}$

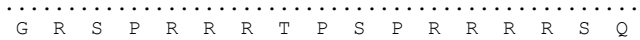

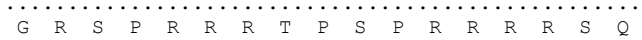

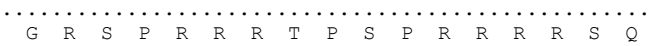
$\begin{array}{lllllllllllllllllllll}G & R & S & P & R & R & R & T & P & S & P & R & R & R & R & S & Q\end{array}$ -AGGCAGGTCCCCTAGAAGAAGAACTCCCTCGCCTCGCAGACGAAGATCTCAA $\begin{array}{lllllllllllllllllll}G & R & S & P & R & R & R & T & P & S & P & R & R & R & R & S & Q\end{array}$ 
$\mathrm{x} 02763$

AH 2-4890

AH 1. I

AH $\quad 4-1835$

AH 4-2015

AH 5-2878

AH 7-1884

AH 7-624

AH 7-640

AH $8-1993$

AH $10-2400$

AH 10-2596

AH 11-2641

AH $12-21$

AH 12-431

CH $1-6126$

CH 10-1982

CH 12-2805

CH 12-82

CH 5-2628

CH $6-444 \mathrm{~A}$

COMPL 4-2971

v01460

X02496

AH 10-1108

AH $\quad$ 10-1612

CH $10-3329$

AH $10-2085$

AH $10-2425$

AH 10-737

AH 10-486

AH 11-1941

AH 11-2336A

AH 11-2514

AH 11-2524

AH 11-2759

AH 11-2866

AH 11-5664

AH 11-965

AH 12-438

AH 3-577

AH 2-2658

AH 3-7991

AH $11-8035$

AH 3-7755

AH 5-920

AH 3-987

AH 4-2307

AH $4-3445$

AH 4-775

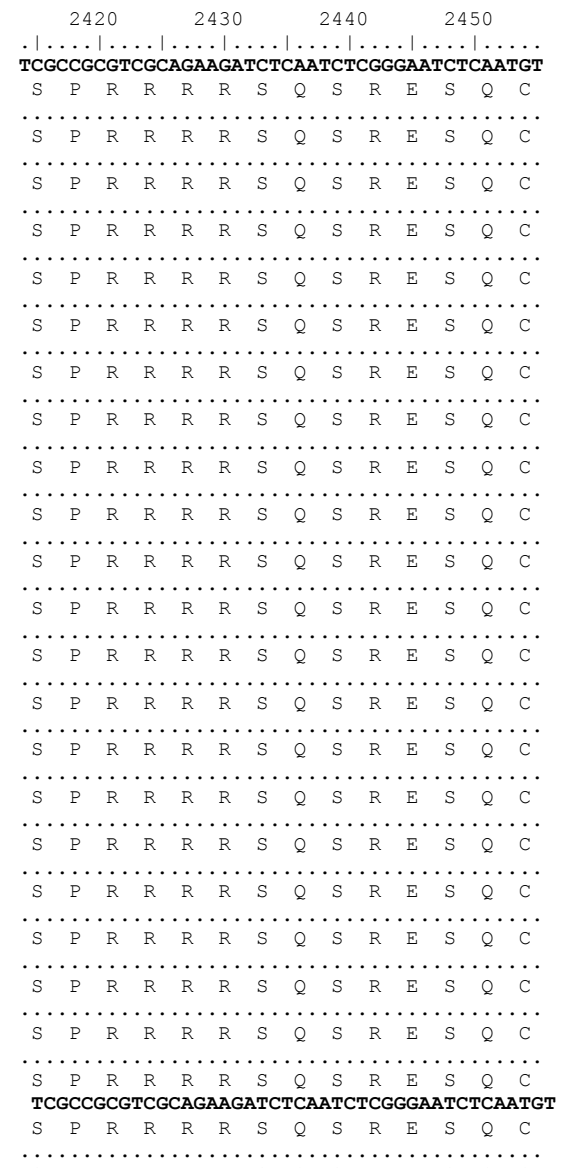

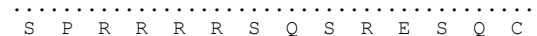

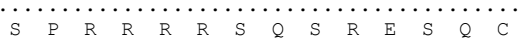

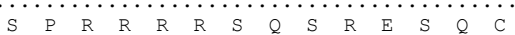

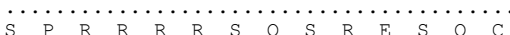

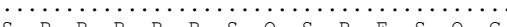

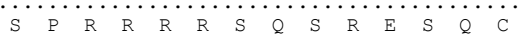

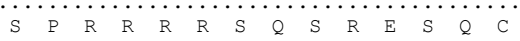

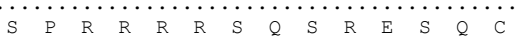

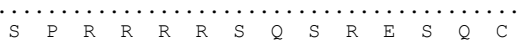

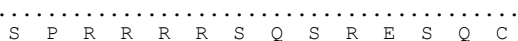

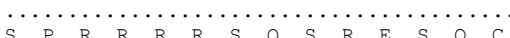

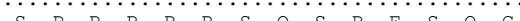

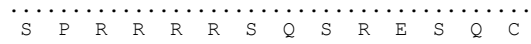

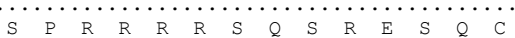

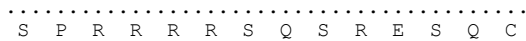

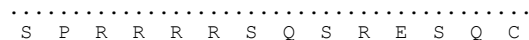

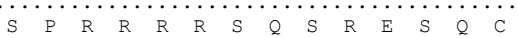

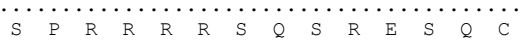

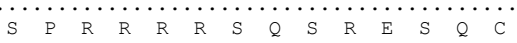

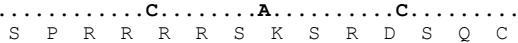

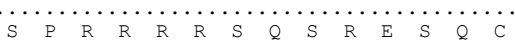

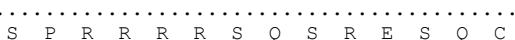

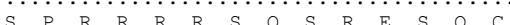

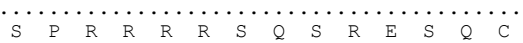

${ }_{S} \cdots_{\dot{P}} \cdots_{R} \cdots_{R} \cdots_{\dot{R}} \cdots_{R} \cdots_{S} \cdots_{Q} \cdots_{S} \cdots_{R} \cdots_{E} \cdots_{S} \cdots_{Q}{ }_{C}$

$\dddot{S}_{\dot{P}}{ }_{R}{ }_{R}{ }_{R}{ }_{R} \cdots_{S} \cdots_{S}{ }_{R}{ }_{E}{ }_{S}{ }_{Q}{ }_{C}$

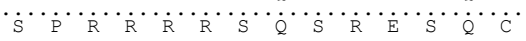




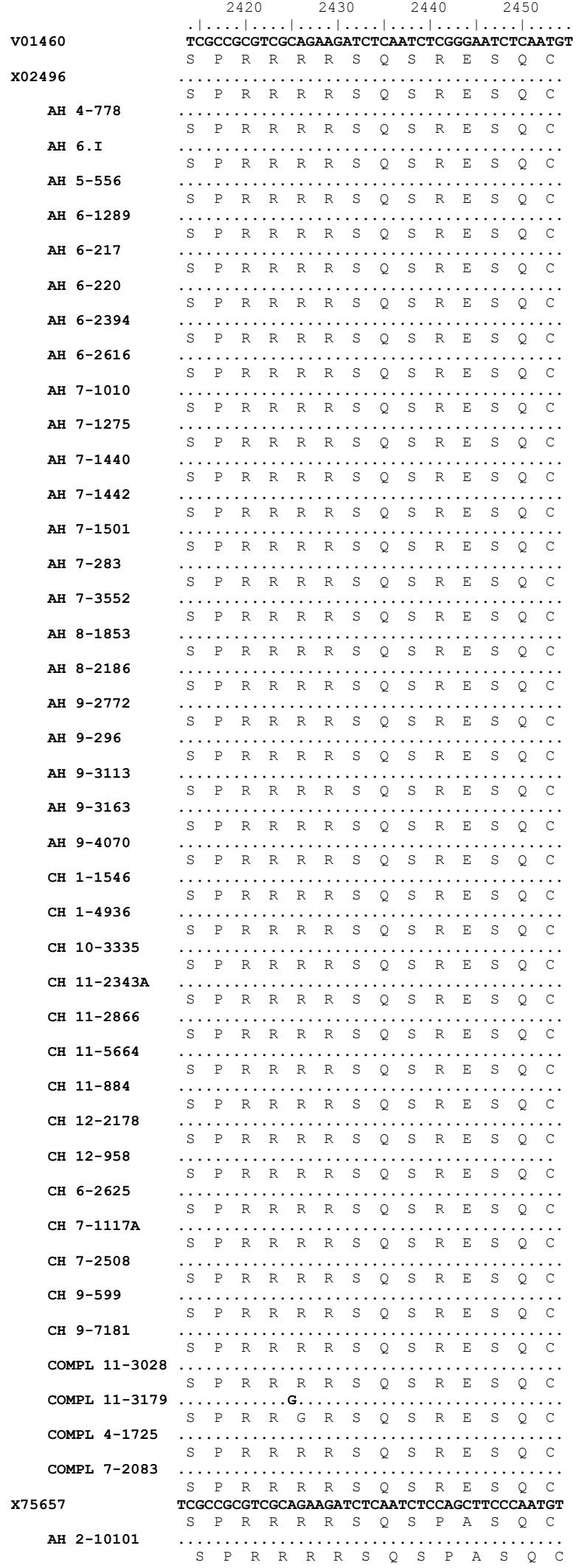


Appendix Part B. Sequences of HBV region preS/S obtained during the study.

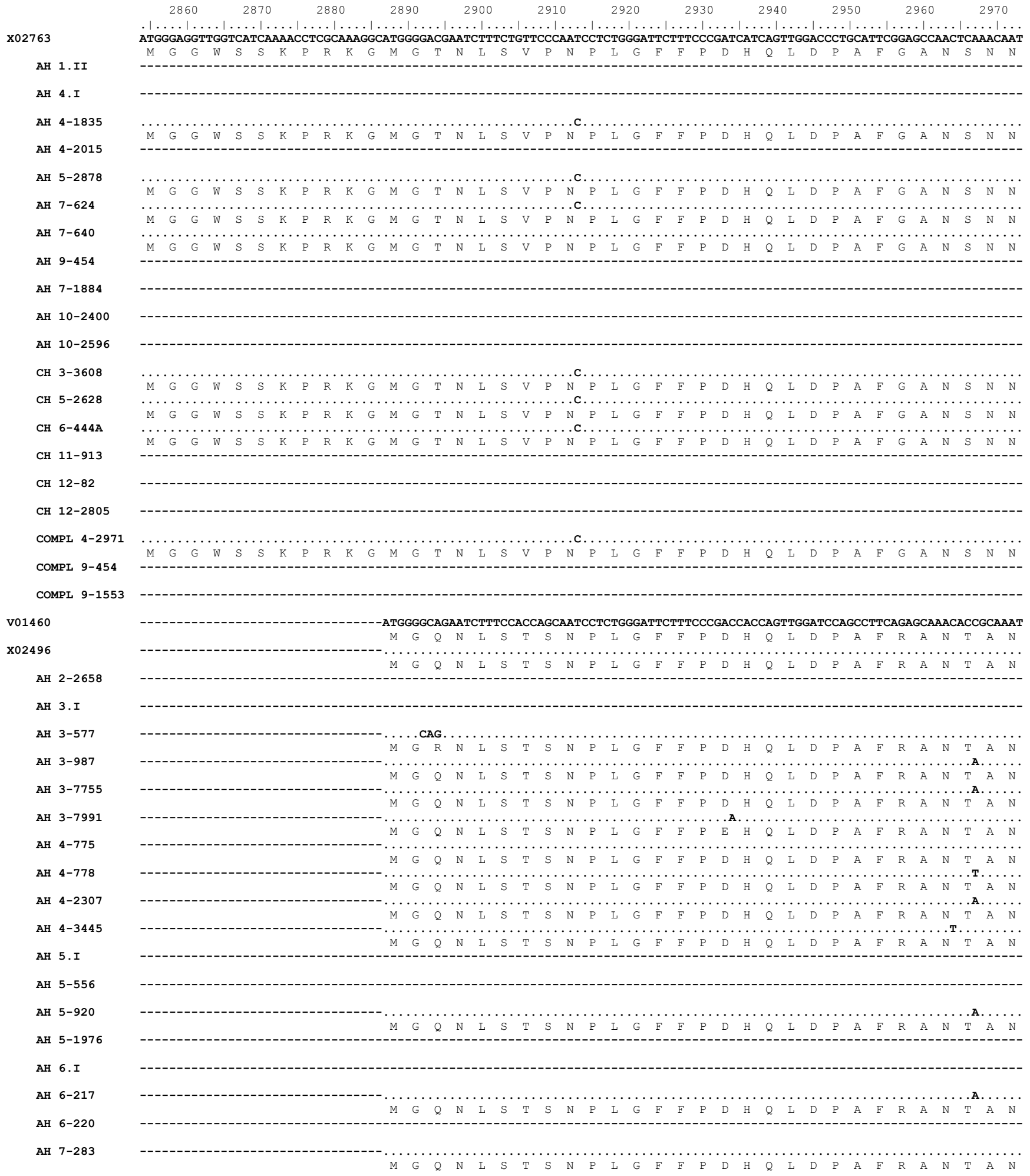


v01460

X02496

AH 7-496

AH 7-1010

AH 7-1275

AH 7-1442

AH $8-2186$

AH 9-2772

AH 9-296

AH 9-297

AH 9-3163

AH $9-3113$

AH 9-3897

AH 9-4070

AH $10-486$

AH 10-737

AH $10-885$

AH $10-1108$

AH 10-1612

AH 10-2085

AH 10-2425

AH 11-965

AH 11-1941

AH $11-2165$

AH 11-2514

AH 11-2759

AH $12-116$

CH 1-1546

CH $\quad 6-2107$

CH 7-1117A

CH $9-599$

CH $11-884$

CH 11-2343A

CH 12-958

COMPL 4-172

COMPL $11-30$

COMPL 11-317

$\mathrm{X} 75657$

AH 2-10101

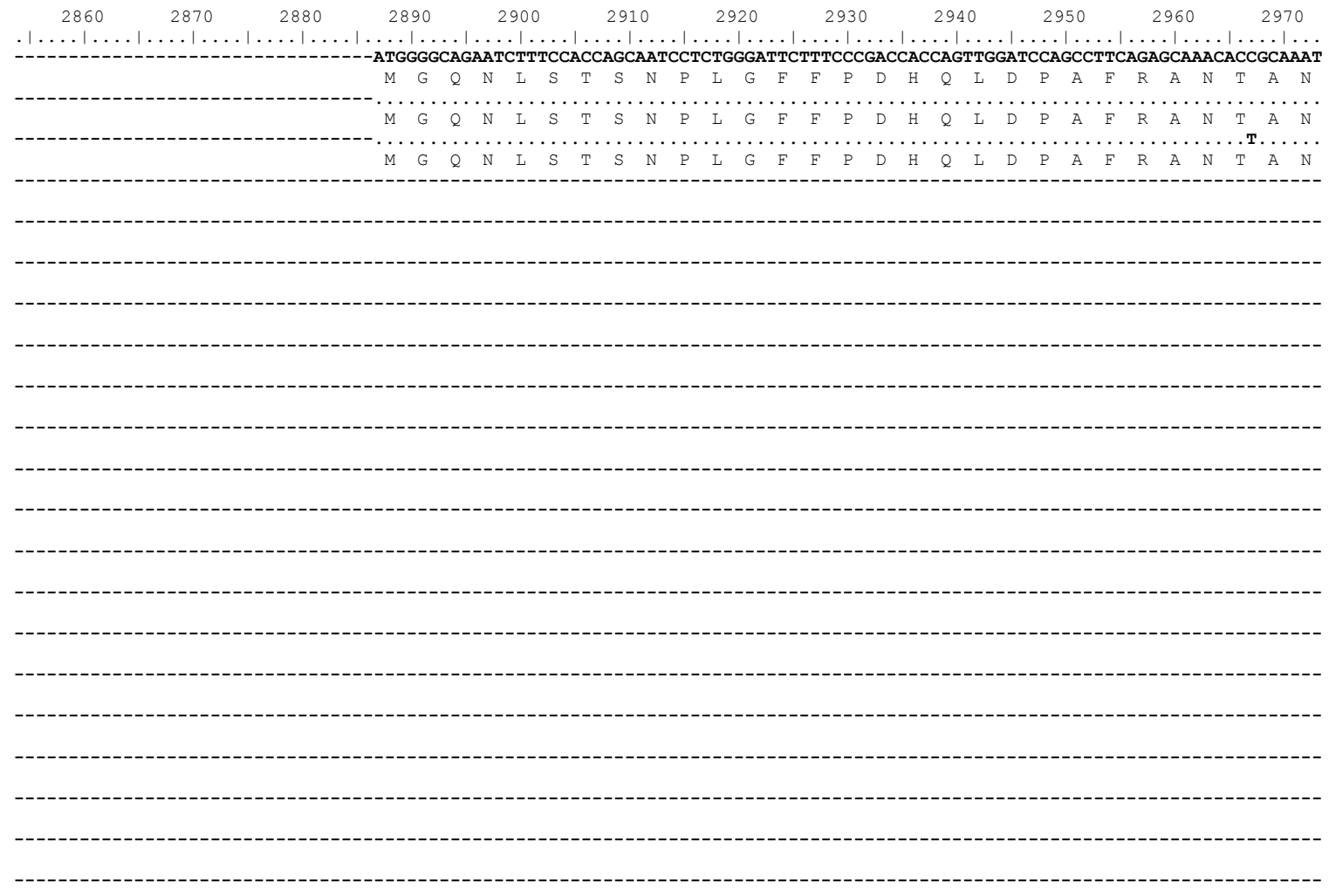

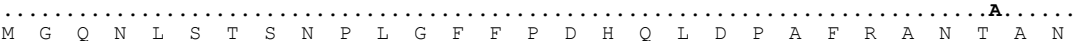

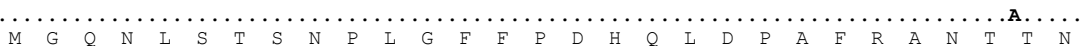

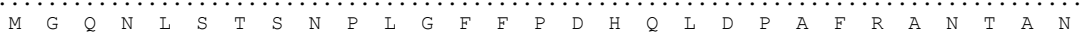

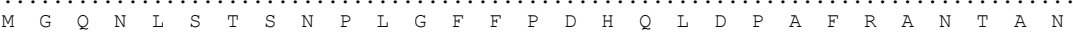

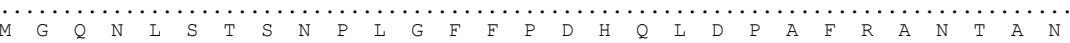

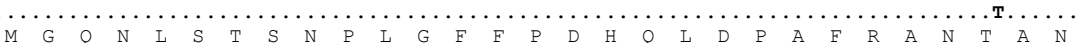
ATGGGGAAGAATATTTCCACCACCAATCCTCTGGGATTTTTTCCCGACCACCAGTTGGATCCAGCATTCAGAGCAAACACCAGAAAT

$\begin{array}{llllllllllllllllllllllllllllllll}M & G & K & N & I & S & T & T & N & P & L & G & F & F & P & D & H & Q & L & D & P & A & F & R & A & N & T & R & N\end{array}$

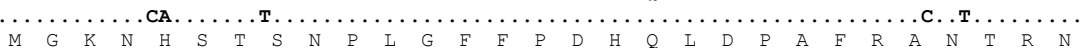


$\mathrm{x} 02763$

AH 1. II

AH 4 . I

AH 4-1835

AH 4-2015

AH 5-2878

AH 7-624

AH 7-640

AH 9-454

AH 7-1884

AH $10-2400$

AH 10-2596

CH 3-3608

CH 5-2628

CH $6-444 \mathrm{~A}$

CH 11-913

CH $12-82$

CH $12-2805$

COMPL 4-2971

COMPL 9-454

COMPL 9-1553

V01460

X02496

AH 2-2658

AH 3 . I

AH 3-577

AH 3-987

AH 3-7755

AH 3-7991

AH 4-775

AH 4-778

AH 4-2307

AH $\quad 4-3445$

AH 5. I

AH 5-556

AH 5-920

AH 5-1976

AH 6.I

AH 6-217

AH $6-220$

AH 7-283

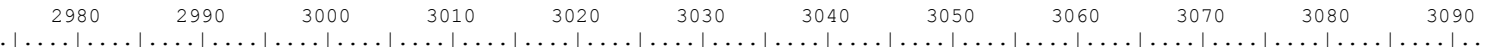
CCAGATTGGGACTTCAACCCCGTCAAGGACGACTGGCCAGCAGCCAACCAAGTAGGAGTGGGAGCATTCGGGCCAAGGCTCACCCCTCCACACGGCGGTATTTTGGGGTGGAGCCCTCAG

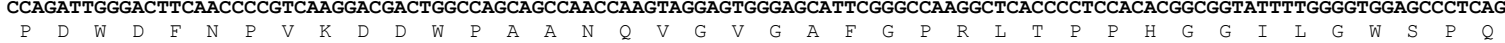

(1)

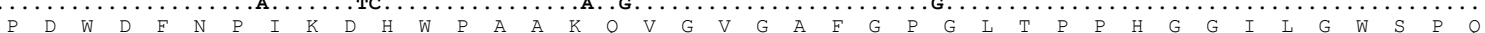
-

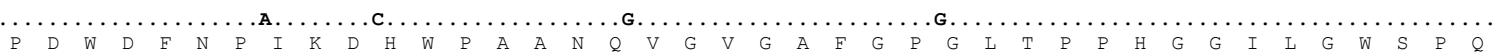

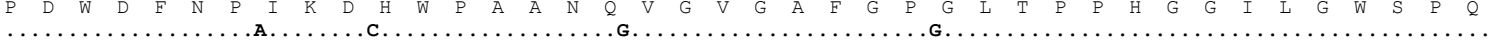

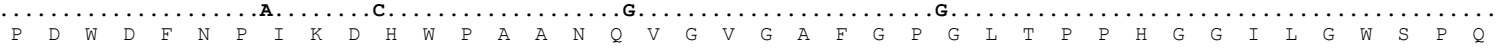

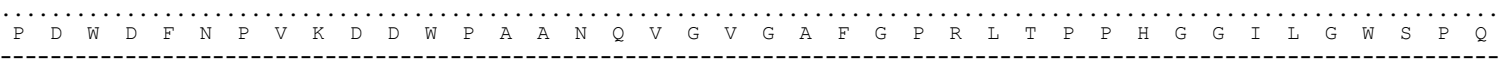

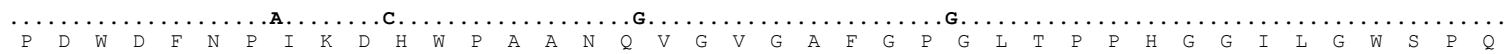

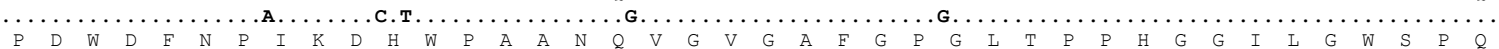

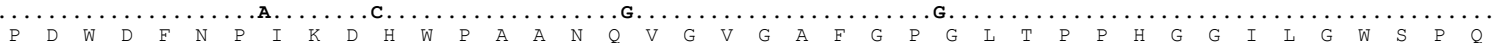
$\ldots \ldots \ldots \ldots \ldots \ldots \ldots . \ldots \ldots \ldots \ldots$
$P$ CCAGATTGGGACTTCAATCCCAACAAGGACACCTGGCCAGACGCCAACAAGGTAGGAGCTGGAGCATTCGGGCTGGGTTTCACCCCACCGCACGGAGGCCTTTTGGGGTGGAGCCCTCAG $\begin{array}{llllllllllllllllllllllllllllllllllllllllllllllllllll}P & D & W & D & F & N & P & N & K & D & T & W & P & D & A & N & K & V & G & A & G & A & F & G & L & G & F & \text { T } & P & P & H & G & G & L & L & G & W & S & P & Q\end{array}$

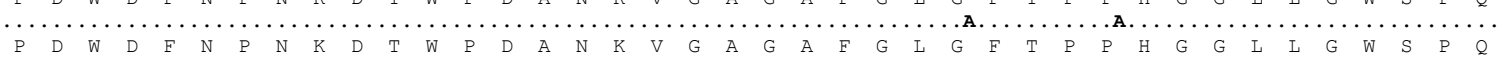

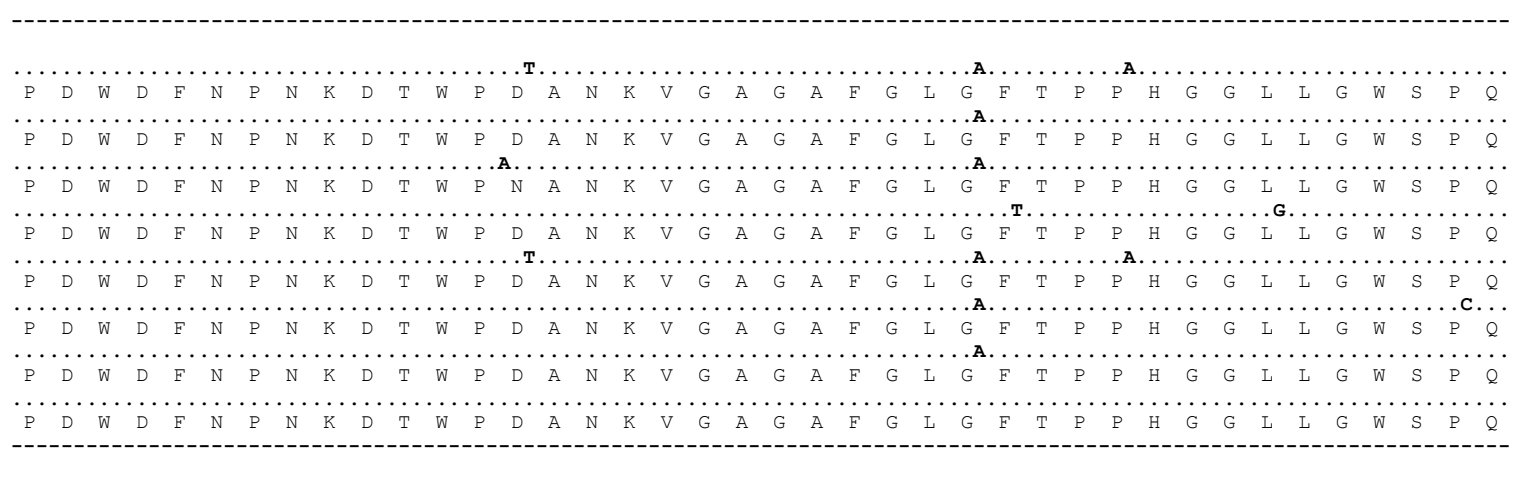

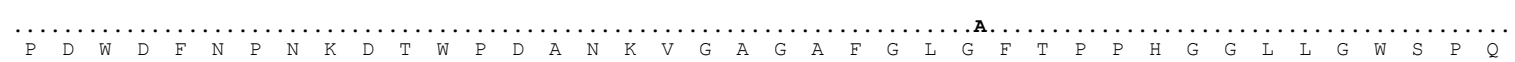

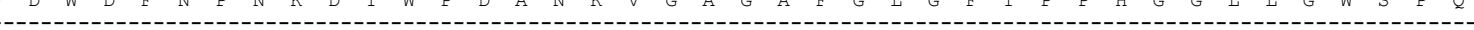

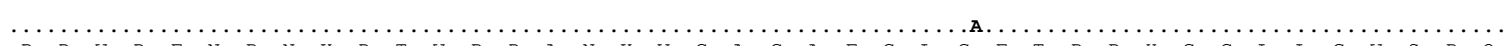

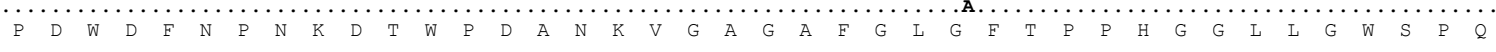

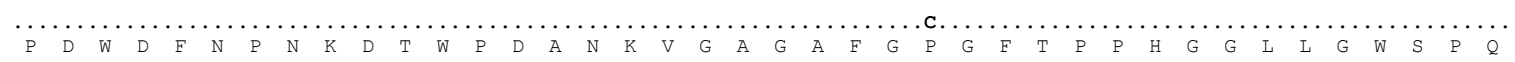


v01460

$\mathrm{x} 02496$

AH 7-496

AH $\quad 7-1010$

AH 7-1275

AH 7-1442

AH $8-2186$

AH $9-2772$

AH $9-296$

AH 9-297

AH 9-3163

AH $9-3113$

AH 9-3897

AH 9-4070

AH $10-486$

AH 10-737

AH $10-885$

AH $10-1108$

AH 10-1612

AH 10-2085

AH $10-2425$

AH 11-965

AH 11-1941

AH 11-2165

AH 11-2514

AH 11-2759

AH 12-116

CH 1-1546

CH $\quad 6-2107$

CH 7-1117A

CH 9-599

CH 11-884

CH 11-2343A

CH 12-958

COMPL 4-1725

COMPL 11-3028

COMPL 11-3179

X75657

AH 2-10101

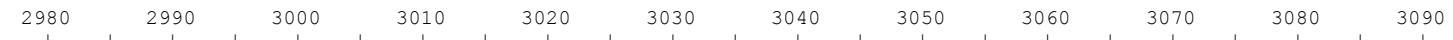
CCAGATTGGGACTTCAATCCCAACAAGGACACCTGGCCAGACGCCAACAAGGTAGGAGCTGGAGCATTCGGGCTGGGTTTCACCCCACCGCACGGAGGCCTTTTGGGGTGGAGCCCTCAG

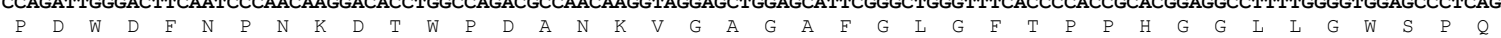

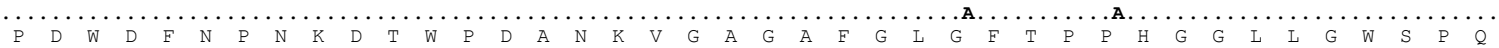

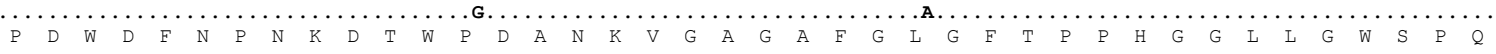


$\mathrm{x} 02763$

AH 1 . II

AH 4 . I

AH 4-1835

AH 4-2015

AH 5-2878

AH 7-624

AH 7-640

AH 9-454

AH 7-1884

AH $10-2400$

AH 10-2596

CH 3-3608

CH 5-2628

CH $6-444 \mathrm{~A}$

CH 11-913

CH $12-82$

CH $12-2805$

COMPL 4-2971

COMPL 9-454

COMPL 9-1553

V01460

X02496

AH 2-2658

AH 3 . I

AH 3-577

AH 3-987

AH 3-7755

AH 3-7991

AH 4-775

AH 4-778

AH 4-2307

AH $\quad 4-3445$

AH 5. I

AH 5-556

AH 5-920

AH 5-1976

AH 6.I

AH 6-217

AH $6-220$

AH 7-283

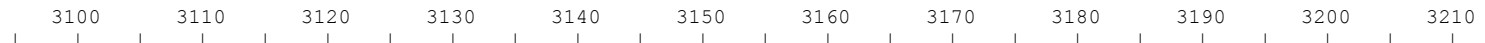
GCTCAGGGATATTGACCACAGTGTCAACAATTCCTCCTCCTGCCTCCACCAATCGGCAGTCAGGAAGGCAGCCTACTCCCATCTCTCCACCTCTAAGAGACAGTCATCCTCAGGCCATG

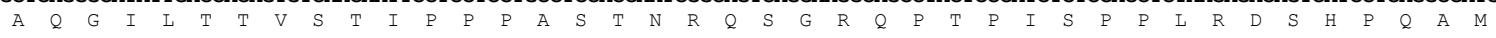

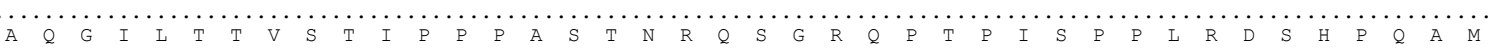

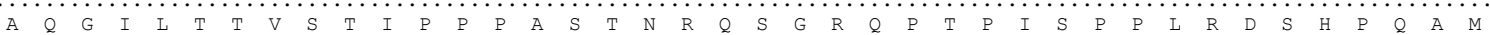

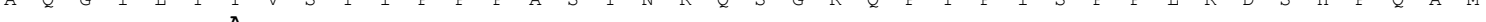

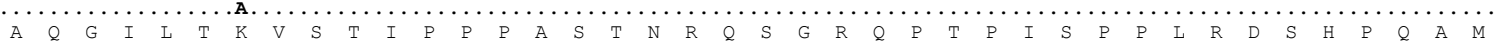

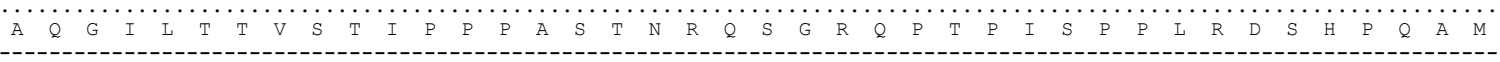

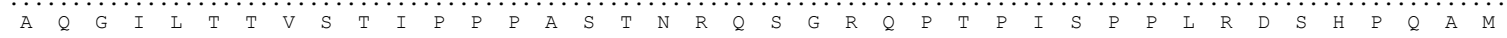

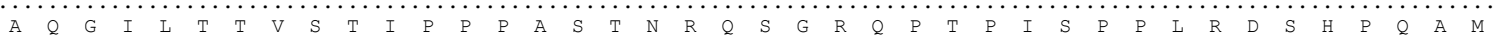

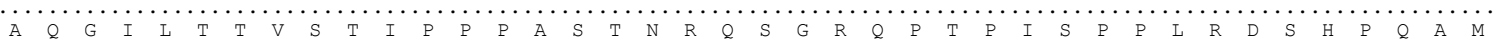
-

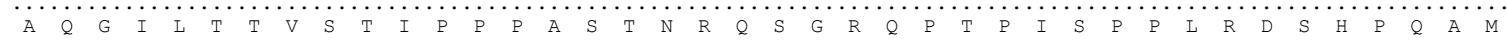

GCTCAGGGCATACTACAAACTTTGCCAGCAAATCCGCCTCCTGCCTCCACCAATCGCCAGTCAGGAAGGCAGCCTACCCCGCTGTCTCCACCTTTGAGAAACACTCATCCTCAGGCCATG $\begin{array}{llllllllllllllllllllllllllllllllllllllllllllllll}A & Q & G & I & L & Q & T & L & P & A & N & P & P & P & A & S & T & N & R & Q & S & G & R & Q & P & T & P & L & S & P & P & L & R & N & T & H & P & Q & A & M & \end{array}$

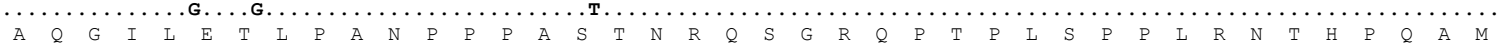

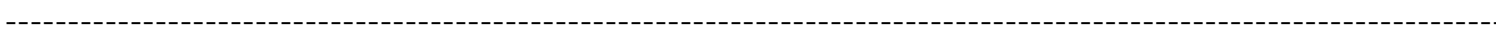

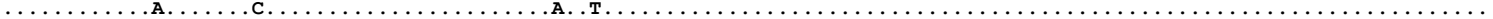

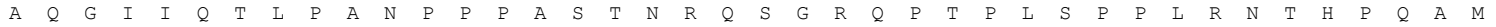

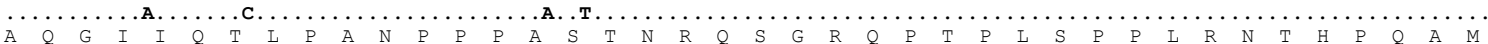

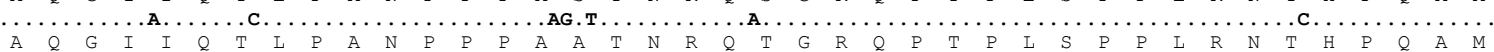

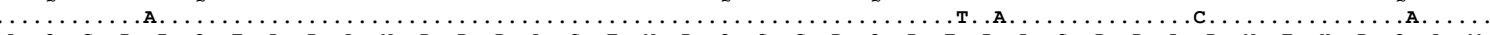

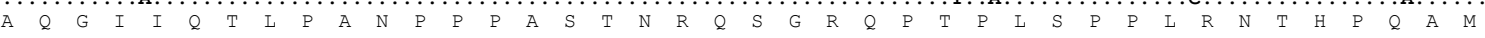

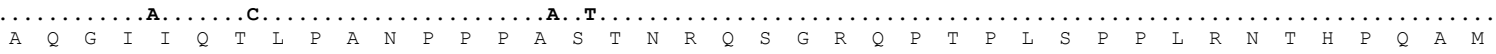

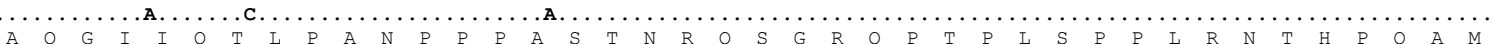

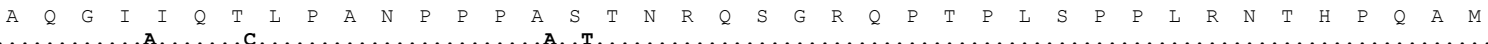

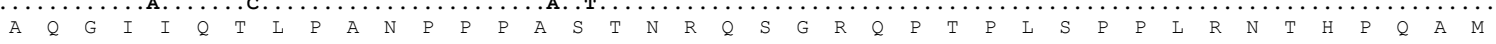

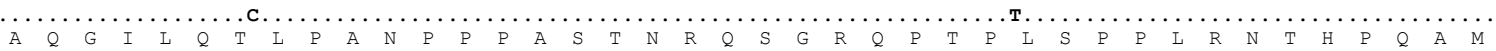
$\begin{array}{lllllllllllllllllllllllllllllllllllllllllllllll}A & Q & G & I & L & Q & T & L & P & A & N & P & P & P & A & S & T & N & R & Q & S & G & R & Q & P & T & P & L & S & P & P & L & R & N & T & H & P & Q & A & M \\ -\end{array}$

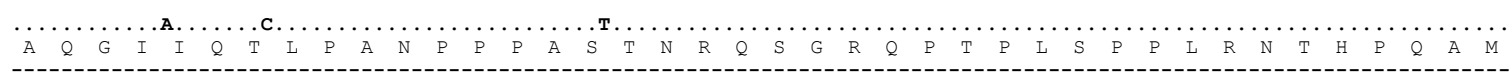

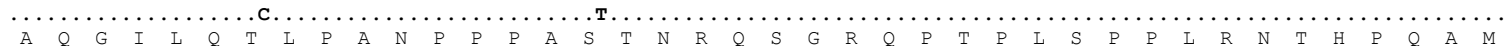

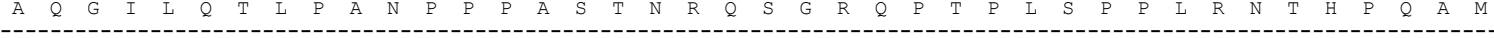

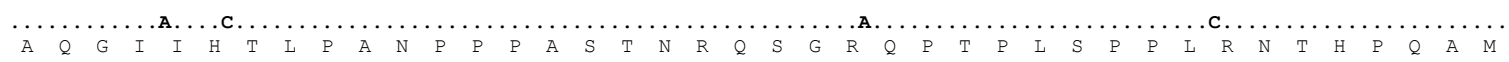


v01460

x02496

AH 7-496

AH $\quad 7-1010$

AH 7-1275

AH 7-1442

AH $8-2186$

AH 9-2772

AH $9-296$

AH 9-297

AH 9-3163

AH $9-3113$

AH 9-3897

AH 9-4070

AH $10-486$

AH 10-737

AH $10-885$

AH $10-1108$

AH 10-1612

AH 10-2085

AH $10-2425$

AH 11-965

AH 11-1941

AH 11-2165

AH 11-2514

AH 11-2759

AH 12-116

CH 1-1546

CH $\quad 6-2107$

CH 7-1117A

CH 9-599

CH 11-884

CH 11-2343A

CH 12-958

COMPL 4-1725

COMPL 11-3028

COMPL 11-3179

X75657

AH 2-10101

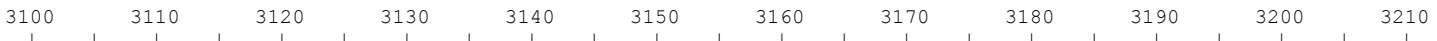
GCTCAGGGATACTACAAACTTTGCCAGCAAATCCGCCTCCTGCCTCCACCAATCGCCAGTCAGGAAGGCAGCCTACCCCGCTGTCTCCACCTTTGAGAAACACTCATCCTCAGGCCATG

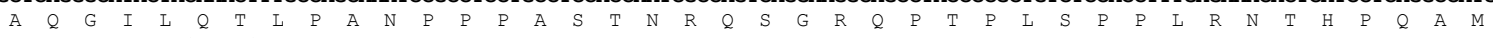

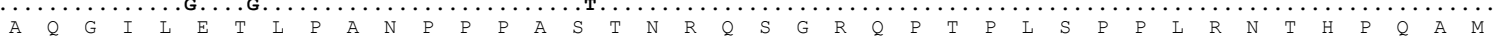

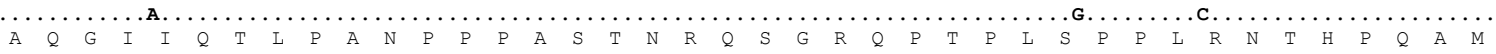


$\times 02763$

AH 1.II

AH 4 . I

AH 4-1835

AH 4-2015

AH 5-2878

AH 7-624

AH 7-640

AH 9-454

AH 7-1884

AH $10-2400$

AH 10-2596

CH 3-3608

CH 5-2628

CH $6-444 \mathrm{~A}$

CH 11-913

CH $12-82$

CH 12-2805

COMPL 4-2971

COMPL 9-454

COMPL 9-1553

V01460

X02496

AH 2-2658

AH 3 . I

AH 3-577

AH 3-987

AH 3-7755

AH 3-7991

AH 4-775

AH 4-778

AH 4-2307

AH $4-3445$

AH 5. I

AH 5-556

AH 5-920

AH 5-1976

AH 6.I

AH 6-217

AH $6-220$

AH 7-283

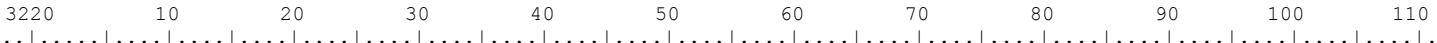
CAGTGGATTCCACTGCCTTCCACCAAACTCTGCAGGATCCCAGAGTCAGGGTCTGTATCTTCCTGCTGGTGGCTCCAGTTCAGGAACAGTAAACCCTGCTCCGAATATTGCCTCTCAC

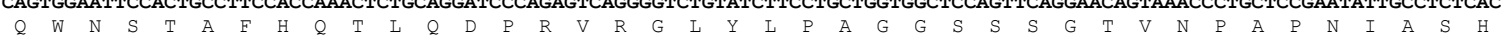

(1)

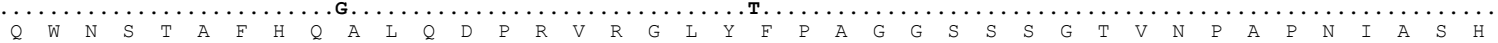
(-

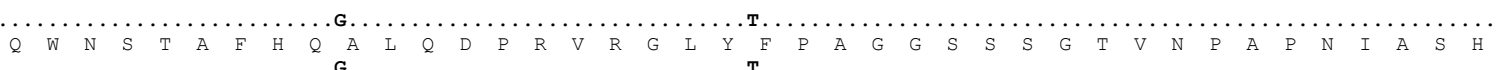

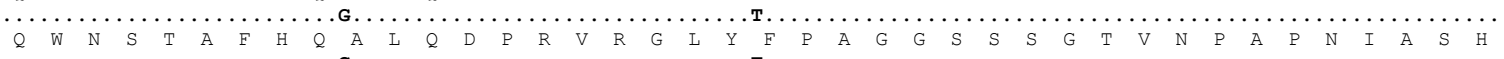

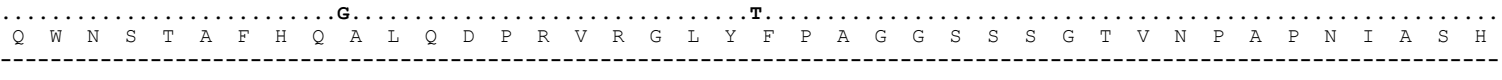

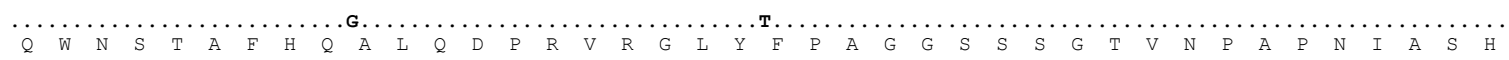

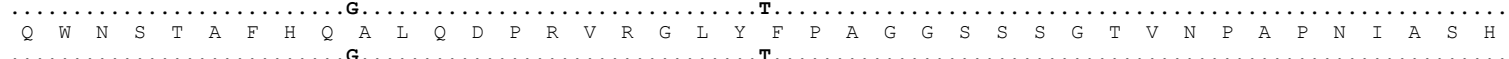

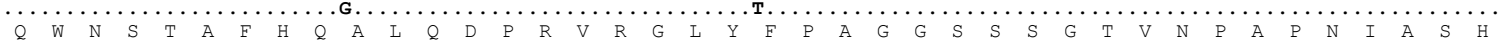

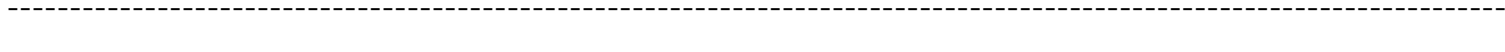

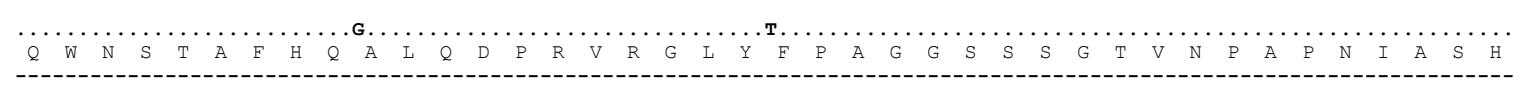
CAGTGGAATTCCACAACCTTCCACCAAACTCTGCAAGATCCCAGAGTGAGAGGCCTGTATTTCCCTGCTGGTGGCTCCAGTTCAGGAACAGTAAACCCTGTTCTGACTACTGCCTCTCCC

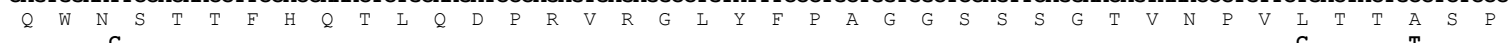

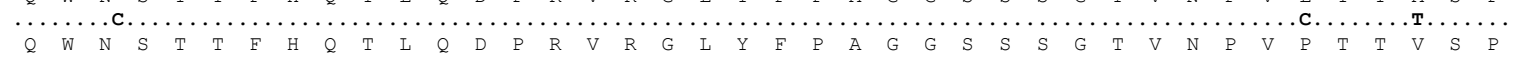

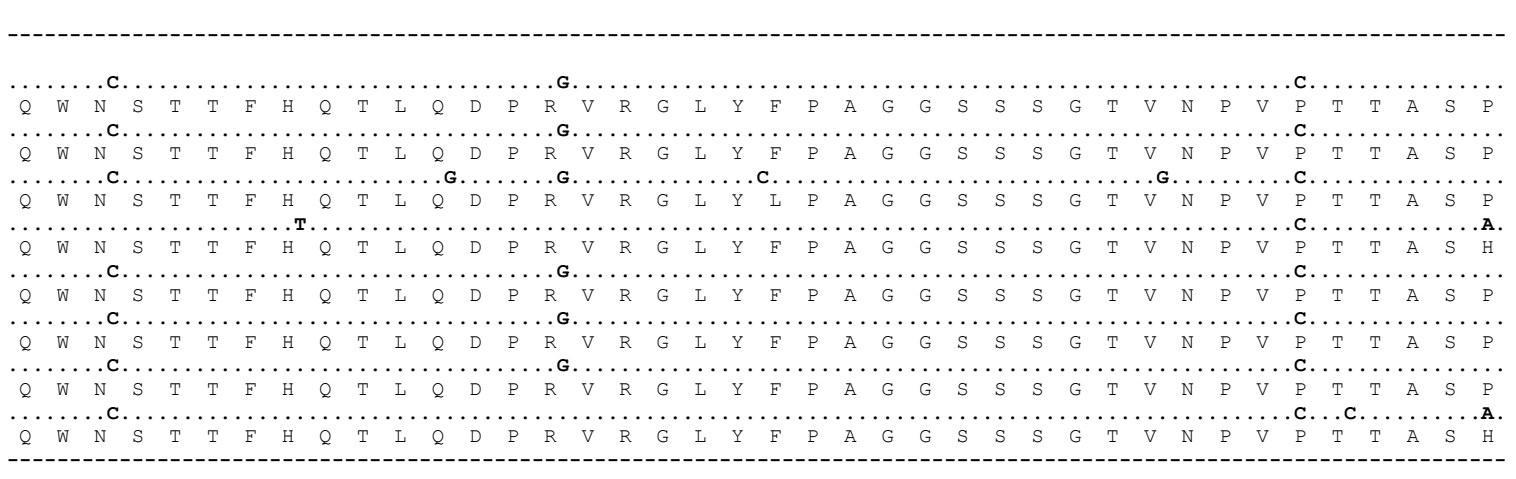

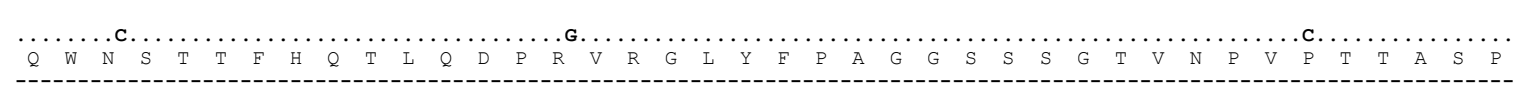

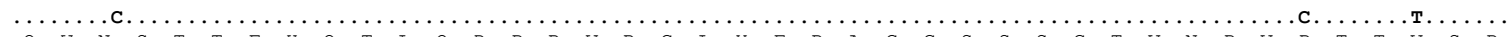

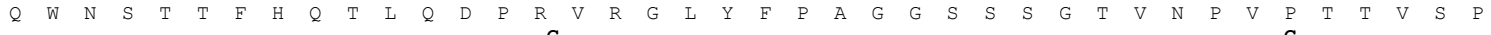

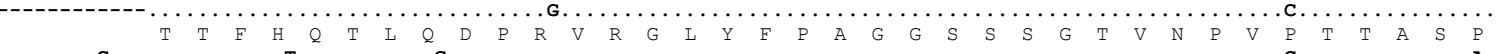

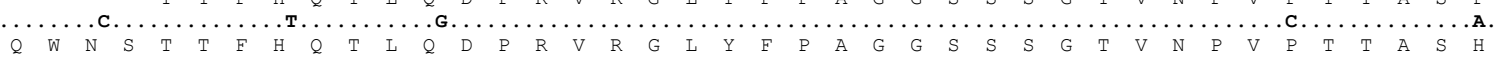


v01460

x02496

AH 7-496

AH 7-1010

AH 7-1275

AH 7-1442

AH $8-2186$

AH 9-2772

AH $9-296$

AH 9-297

AH 9-3163

AH $9-3113$

AH 9-3897

AH 9-4070

AH $10-486$

AH 10-737

AH $10-885$

AH $10-1108$

AH 10-1612

AH 10-2085

AH $10-2425$

AH 11-965

AH 11-1941

AH 11-2165

AH 11-2514

AH 11-2759

AH 12-116

CH 1-1546

CH $\quad 6-2107$

CH 7-1117A

CH 9-599

$\mathrm{CH} \quad 11-884$

CH 11-2343A

CH 12-958

COMPL 4-1725

COMPL 11-3028

COMPL $11-3179$

X75657

AH 2-10101

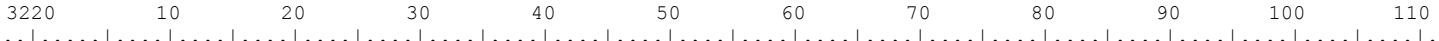
CAGTGGATTCCACAACCTTCCACCAAACTCTGCAAGATCCAGAGTGAGAGGCCTGTATTTCCCTGCTGGTGGCTCCAGTTCAGGAACAGTAAACCCTGTTCTGACTACTGCCTCTCCC $\begin{array}{llllllllllllllllllllllllllllllllllllllllllllllllll}Q & W & N & S & T & T & F & H & Q & T & L & Q & D & P & R & V & R & G & L & Y & F & P & A & G & G & S & S & S & G & T & V & N & P & V & L & T & T & A & S & P\end{array}$ $Q$
$W$

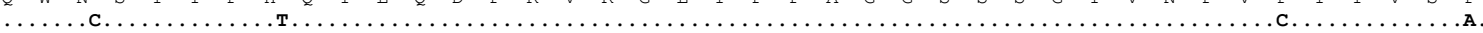

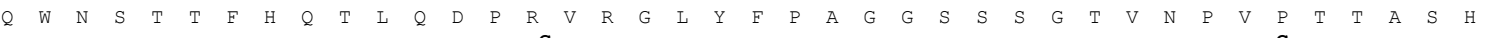

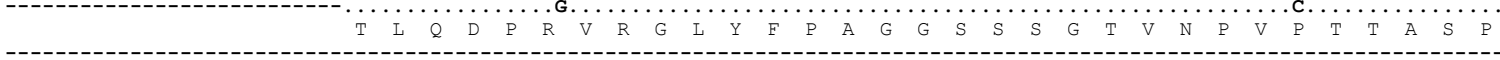

$-$

$-$

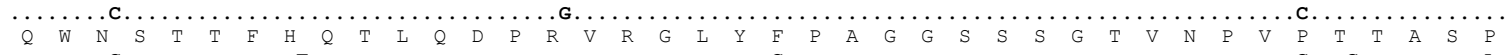

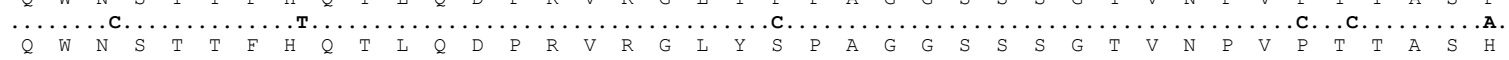

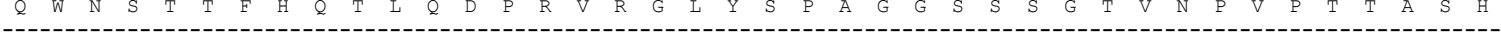
$\ldots \ldots$
$Q$
$Q$

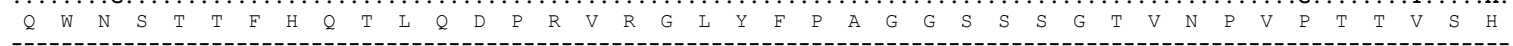
(1)

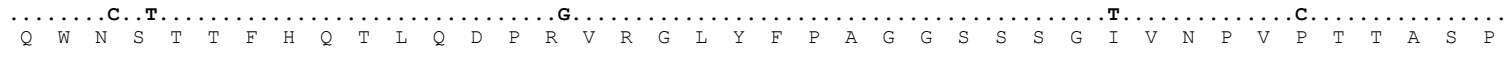

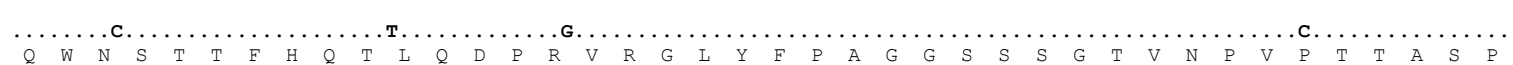
CAGTGGAATTCCACAACATTCCACCAAGCTCTGCAGGATCCCAGAGTAAGAGGCCTGTATTTTCCTGCTGGTGGCTCCAGTTCCGGAACAGTGAACCCTGTTCCGACTACTGCCTCACTC $\begin{array}{lllllllllllllllllllllllllllllllllllllllll}Q & W & N & S & T & T & F & H & Q & A & L & Q & D & P & R & V & R & G & L & Y & F & P & A & G & G & S & S & S & G & T & V & N & P & V & P & T & T & A & S & L\end{array}$

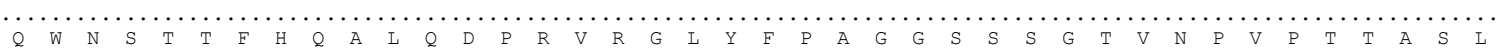


$\mathrm{x} 02763$

AH 1. II

AH 4 . I

AH 4-1835

AH 4-2015

AH 5-2878

AH 7-624

AH 7-640

AH 9-454

AH 7-1884

AH $10-2400$

AH $10-2596$

CH 3-3608

CH 5-2628

CH $6-444 \mathrm{~A}$

CH 11-913

CH 12-82

CH $12-2805$

COMPL 4-2971

COMPL 9-454

COMPL 9-1553

V01460

X02496

AH 2-2658

AH 3.I

AH 3-577

AH 3-987

AH 3-7755

AH 3-7991

AH 4-775

AH 4-778

AH $\quad 4-2307$

AH $\quad 4-3445$

AH 5.I

AH 5-556

AH 5-920

AH $5-1976$

AH 6.I

AH $\quad 6-217$

AH $6-220$

AH $7-283$

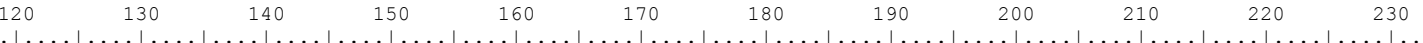
ATCTCGTCAATCTCCGCGAGGACTGGGGACCCTGTGACGAACATGGAGAACATCACATCAGGATTCCTAGGACCCCTGCTCGTGTTACAGGCGGGTTTTTCTTGTTGACAAGAATCCTC

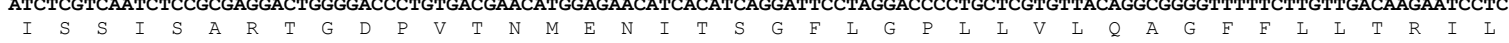

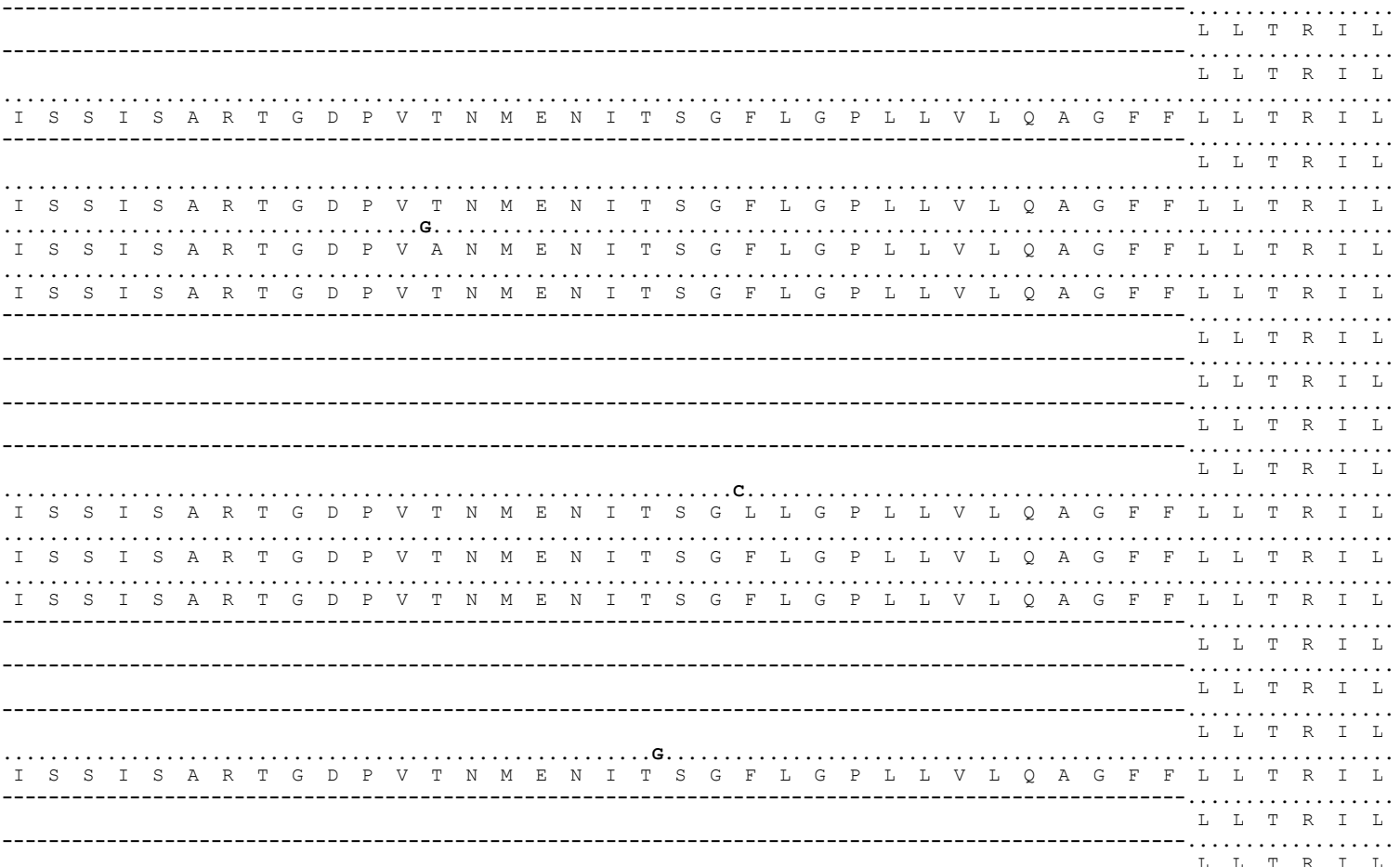

TTATCGTCAATCTTCTCGAGGATTGGGGACCCTGCGCTGAACATGGAGAACATCACATCAGGATTCCTAGGACCCCTTCTCGTGTTACAGGCGGGTTTTTCTTGTTGACAAGAATCCTC $\begin{array}{llllllllllllllllllllllllllllllllllllllllll}L & S & S & I & F & S & R & I & G & D & P & A & L & N & M & E & N & I & T & S & G & F & L & G & P & L & L & V & L & Q & A & G & F & F & L & L & T & R & I & L\end{array}$

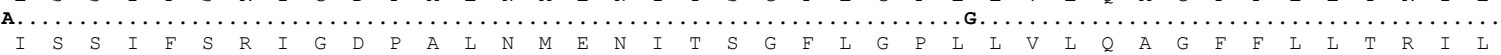

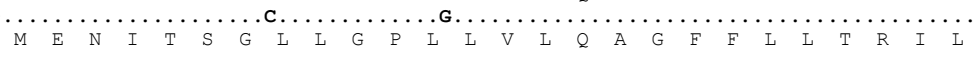
$\begin{array}{llllll} & L & T & R & I & L\end{array}$

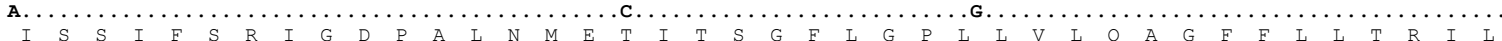

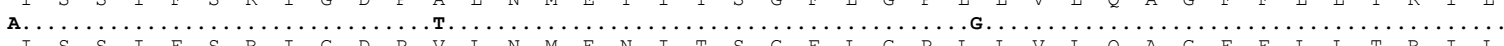

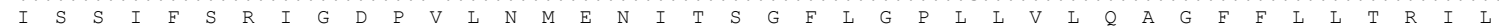

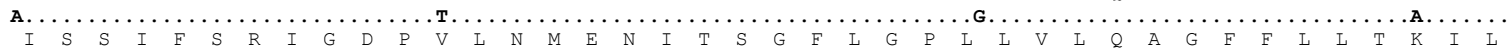

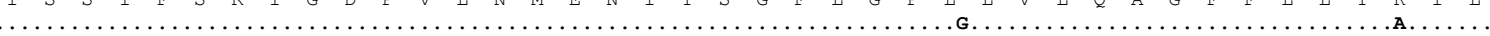

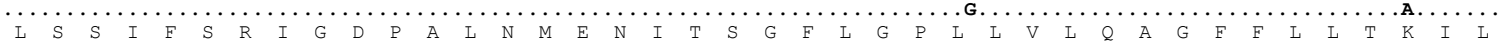

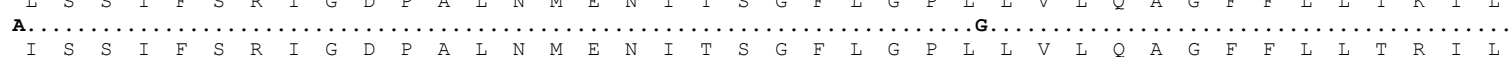

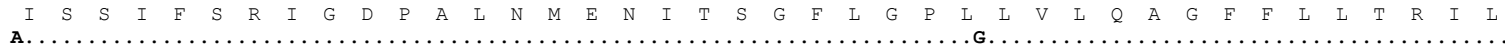

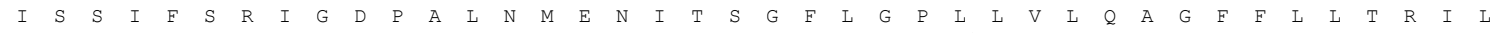

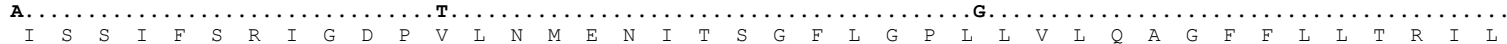

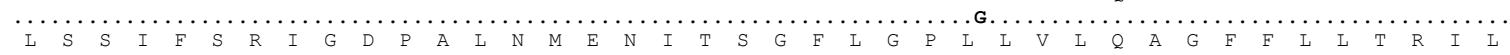

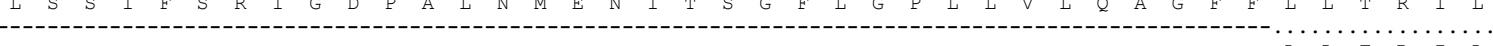

L $L$ T $\quad$ R $\quad$ I

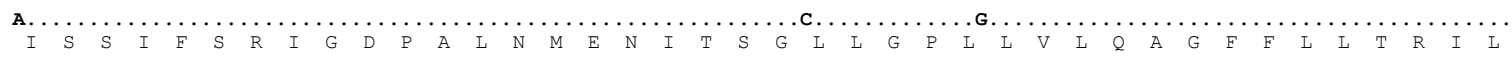

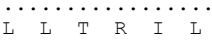
$\begin{array}{lllllll} & L & T & R & I & L\end{array}$

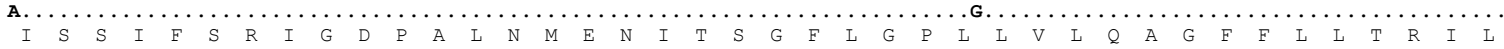

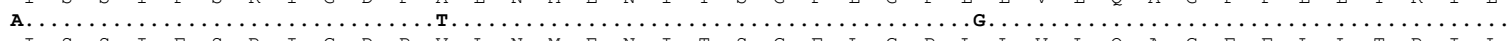

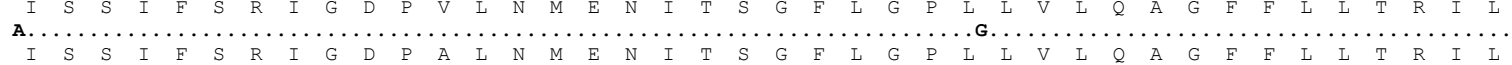


v01460

x02496

AH 7-496

AH 7-1010

AH 7-1275

AH 7-1442

AH $8-2186$

AH 9-2772

AH 9-296

AH $\quad$ 9-297

AH 9-3163

AH $9-3113$

AH 9-3897

AH 9-4070

AH $10-486$

AH $10-737$

AH $10-885$

AH $10-1108$

AH 10-1612

AH 10-2085

AH $10-2425$

AH 11-965

AH 11-1941

AH $11-2165$

AH 11-2514

AH 11-2759

AH 12-116

CH $1-1546$

CH 6-2107

CH 7-1117A

CH 9-599

CH 11-884

CH 11-2343A

CH 12-958

COMPL 4-1725 COMPL 11-3028

COMPL 11-3179

X75657

AH 2-10101

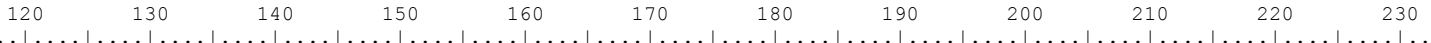
TTATCGTCAATCTTCTCGAGGATTGGGGACCCTGCGCTGAACATGGAGAACATCACATCAGGATTCCTAGGACCCCTTCTCGTGTTACAGGCGGGTTTTTCTTGTTGACAAGAATCCTC $\begin{array}{lllllllllllllllllllllllllllllllllllllllllllllll}L & S & S & I & F & S & R & I & G & D & P & A & L & N & M & E & N & I & T & S & G & F & L & G & P & L & L & V & L & Q & A & G & F & F & L & L & T & R & I & L\end{array}$

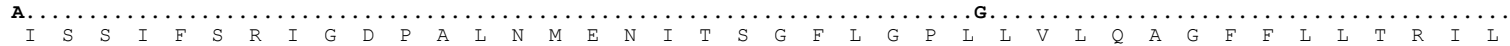

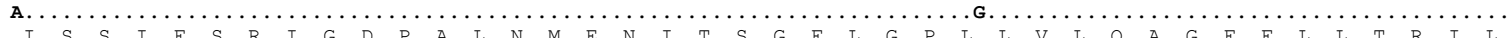

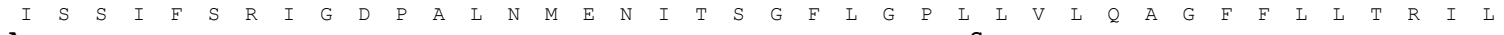

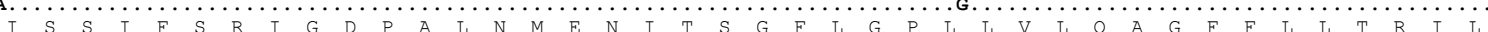
\begin{tabular}{l}
$S T H$ \\
\hline
\end{tabular} $\begin{array}{llllll} & L & T & R & I & L\end{array}$

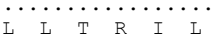
$\begin{array}{llllll} & L & T & R & I & L\end{array}$ $\begin{array}{lllll} & L & T & R & I\end{array}$ $\ldots \ldots \ldots \ldots \ldots$ $\begin{array}{llllll} & L & T & R & \ldots & \ldots\end{array}$ $\begin{array}{llllll} & L & T & R & I & \end{array}$ $\begin{array}{llllll}L & L & T & R & I & L\end{array}$

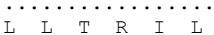

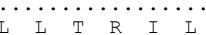
$\ldots \ldots \ldots \ldots \ldots \ldots$

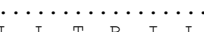
$\begin{array}{llllll} & L & T & R & I & L\end{array}$

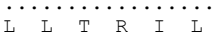

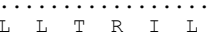

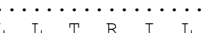
$\ldots \ldots \ldots \ldots \ldots$ ......................

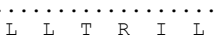

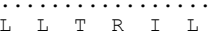
$\begin{array}{lllll} & L & T & R & I\end{array}$ $A$
$I$

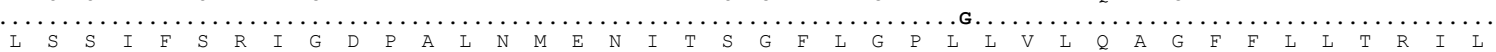

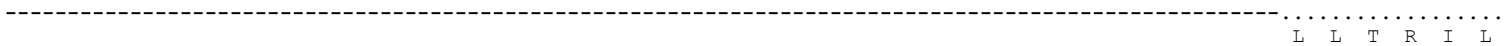

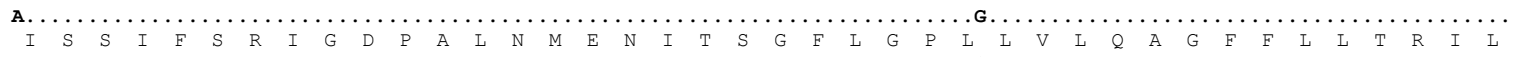

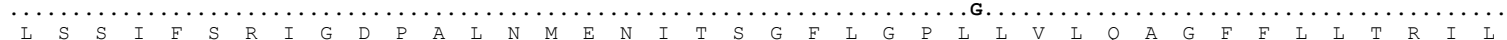
$\cdots \ldots \ldots \ldots$

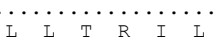

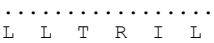

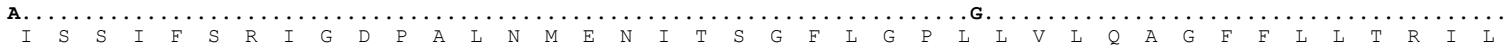

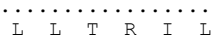
$A$
$I$ ATCTCGTCAATCTTCTCGAGGATTGGGGACCCTGCACCGAACATGGAAAGCATCACATCAGGATTCCTAGGACCCCTGCTCGTGTTACAGGCGGGGTTTTTCTTGTTGACAAAAATCCTC $\begin{array}{lllllllllllllllllllllllllllllllllllllllllllllllllllll}I & S & S & I & F & S & R & I & G & D & P & A & P & N & M & E & S & I & T & S & G & F & L & G & P & L & L & V & L & Q & A & G & F & F & L & L & T & K & I & L\end{array}$

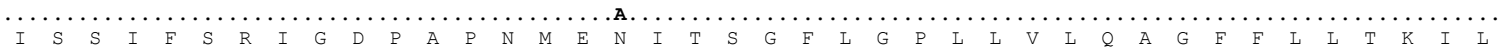


$\mathrm{X} 02763$

AH 1.II

AH 4 . I

AH 4-1835

AH 4-2015

AH 5-2878

AH 7-624

AH 7-640

AH 9-454

AH $7-1884$

AH $10-2400$

AH 10-2596

CH 3-3608

CH 5-2628

CH $6-444 \mathrm{~A}$

CH 11-913

CH 12-82

CH $12-2805$

COMPL 4-2971

COMPL 9-454

COMPL 9-1553

V01460

X02496

AH 2-2658

AH 3.I

AH 3-577

AH 3-987

AH 3-7755

AH 3-7991

AH 4-775

AH 4-778

AH 4-2307

AH $\quad 4-3445$

AH 5.I

AH 5-556

AH 5-920

AH 5-1976

AH $6 . \mathrm{I}$

AH $6-217$

AH $6-220$

AH $7-283$ $\begin{array}{lllllllllll}240 & 250 & 260 & 270 & 280 & 290 & 300 & 310 & 320 & 330 & 340\end{array}$ ACAATACCGCAGAGTCTAGACTCGTGGTGGACTTCTCTCAATTTTCTAGGGGATCTCCCGTGTGTCTTGGCCAAAATTCGCAGTCCCCAACCTCCAATCACTCACCAACCTCCTGTCCT

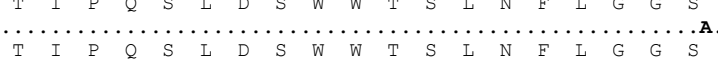

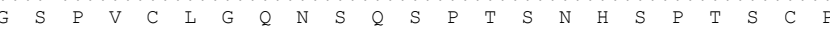

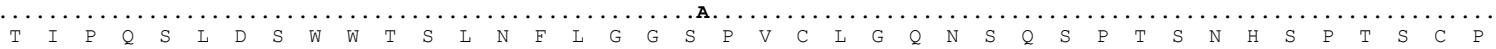

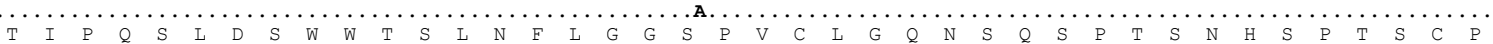

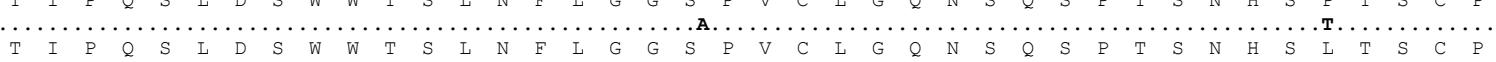

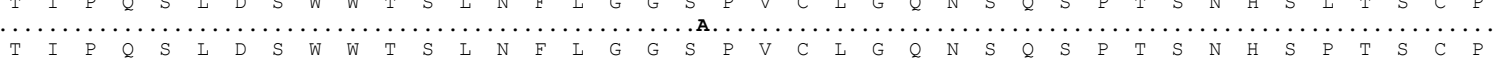

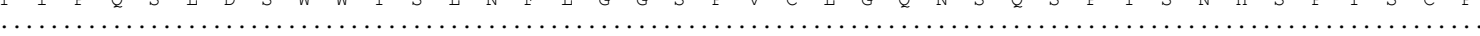

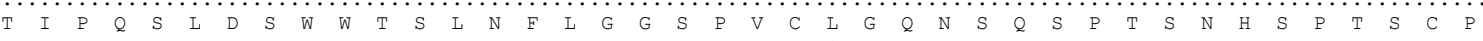

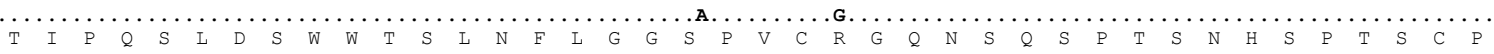

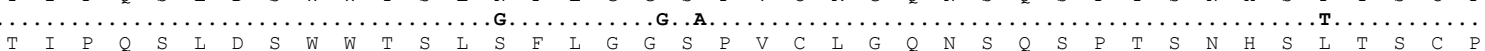

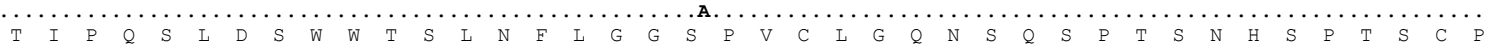

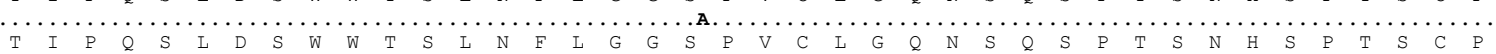

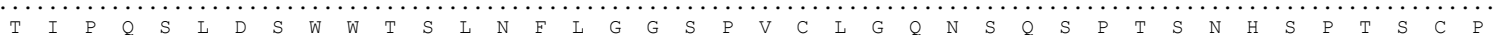

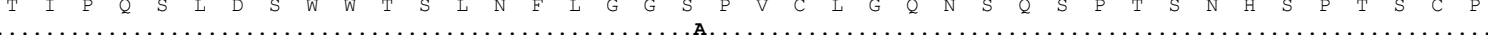

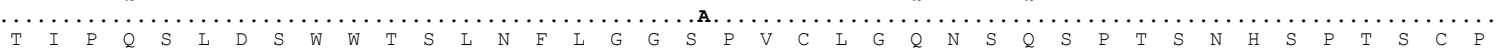

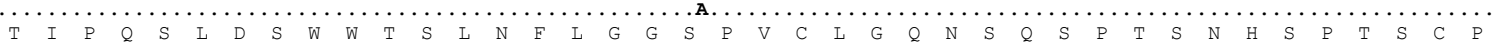

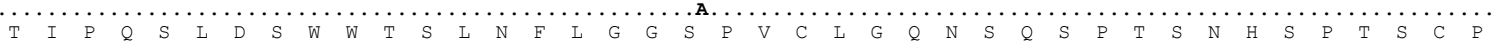

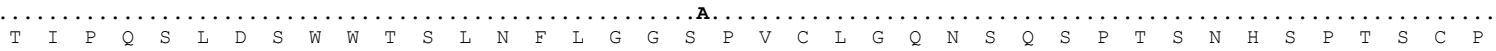

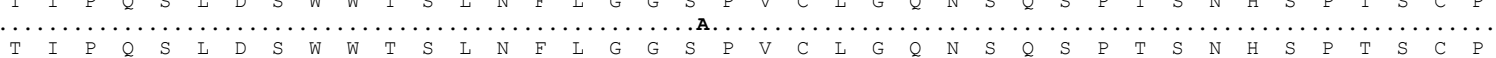

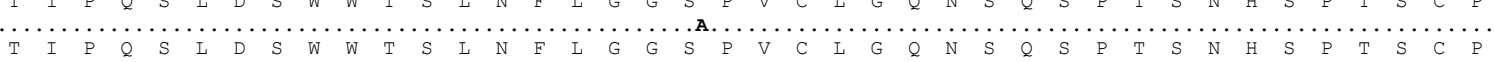

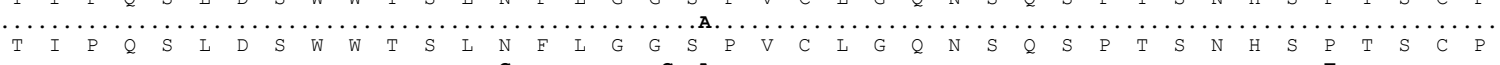

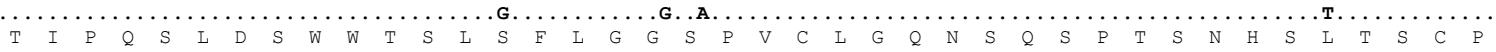

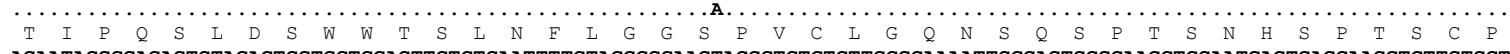
ACAATACCGCAGAGTCTAGACTCGTGGTGGACTTCTCTCAATTTTCTAGGGGGAACTACCGTGTGTCTTGGCCAAAATTCGCAGTCCCCAACCTCCAATCACTCACCAACCTCTTGTCCT

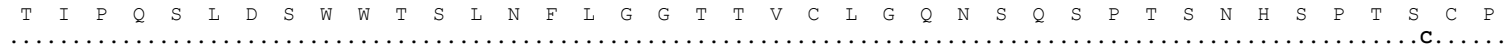

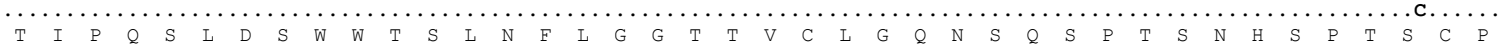

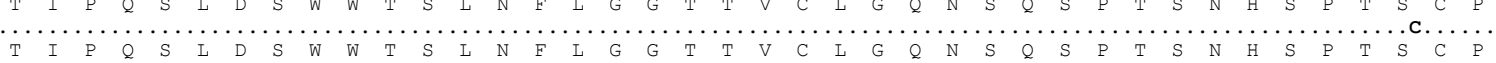

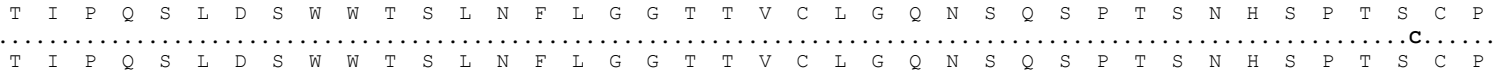

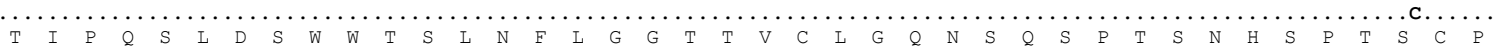

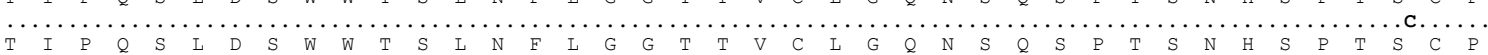

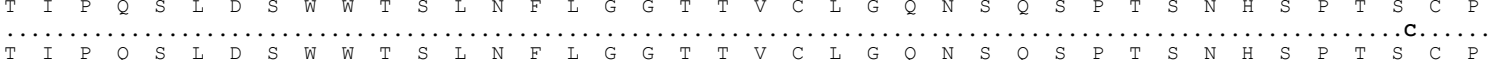

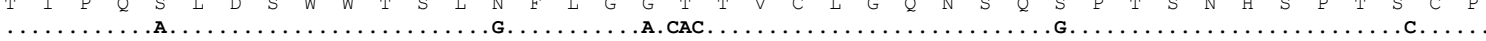
$T$
$T$

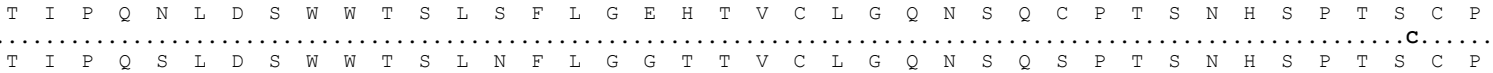
$T$
$T$

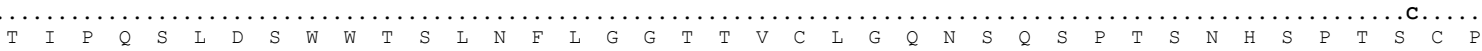

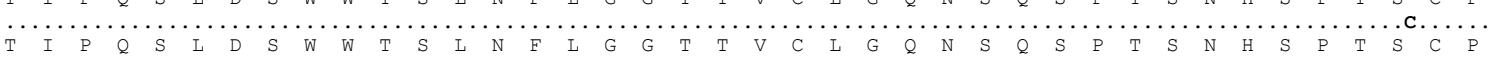

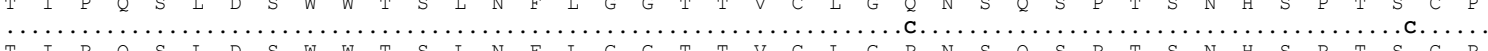

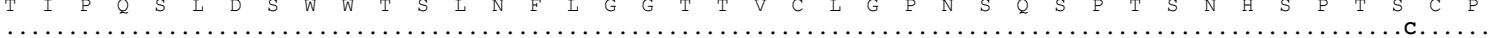

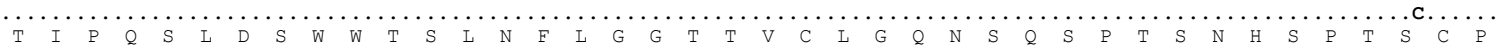
$T$
$T$

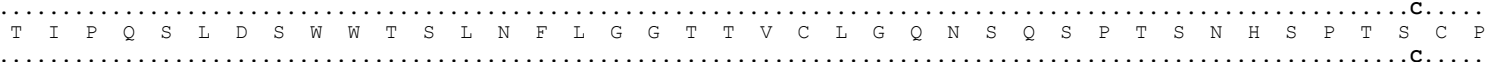

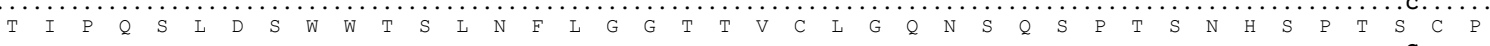

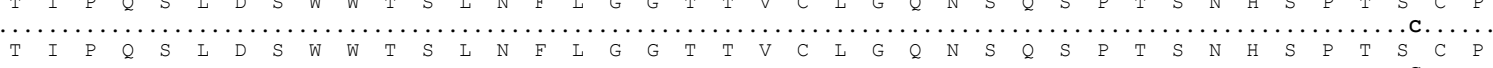

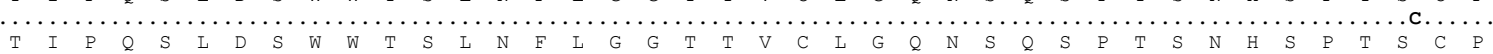

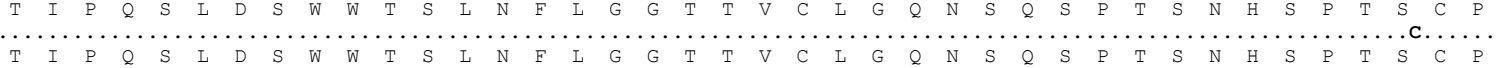


v01460

x02496

AH 7-496

AH 7-1010

AH 7-1275

AH 7-1442

AH $8-2186$

AH 9-2772

AH 9-296

AH 9-297

AH $9-3163$

AH 9-3113

AH 9-3897

AH 9-4070

AH $10-486$

AH 10-737

AH $10-885$

AH $10-1108$

AH 10-1612

AH 10-2085

AH $10-2425$

AH 11-965

AH 11-1941

AH 11-2165

AH 11-2514

AH 11-2759

AH 12-116

CH 1-1546

CH 6-2107

CH 7-1117A

CH 9-599

CH 11-884

CH 11-2343A

CH 12-958

COMPL 4-1725

COMPL 11-3028

COMPL 11-3179

X75657

AH 2-10101

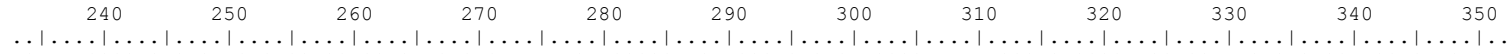

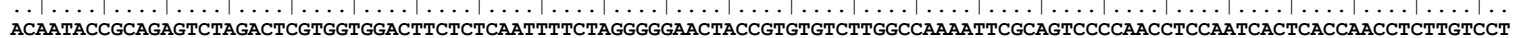

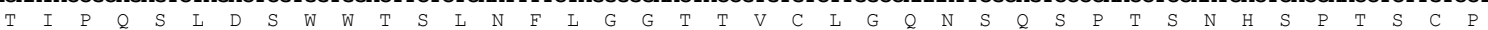

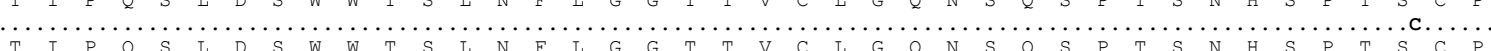

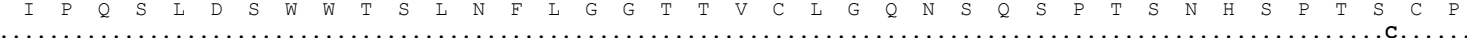

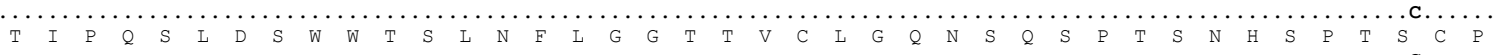

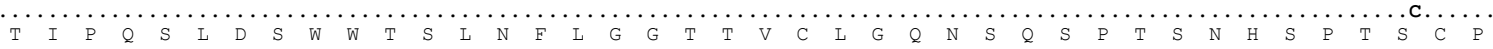

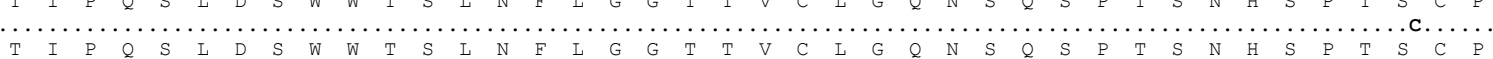

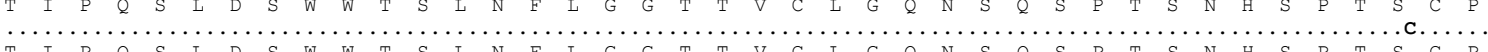
$\begin{array}{llllllllllllllllll}2 & N & S & Q & S & P & T & S & N & H & S & P & T & S & C & P\end{array}$

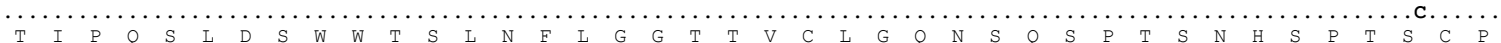

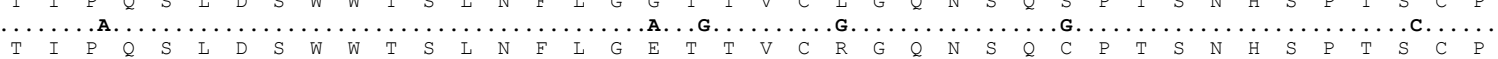
$\cdots$
$T$

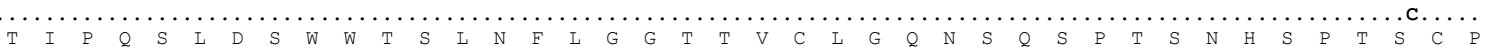

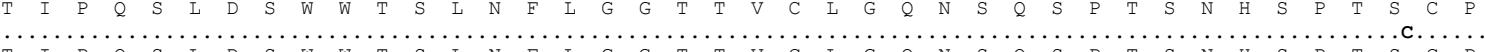

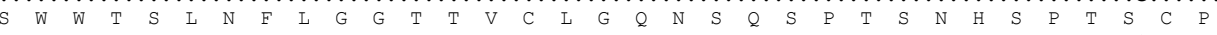

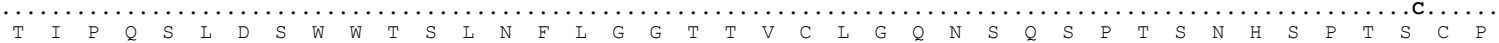

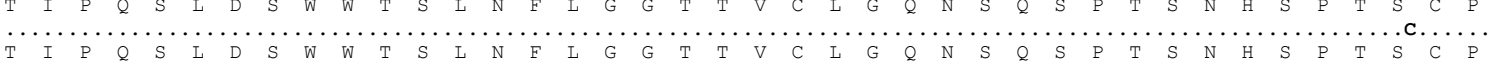

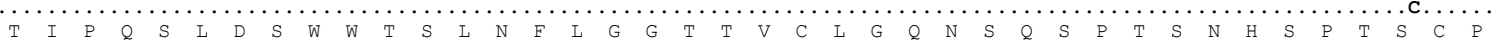

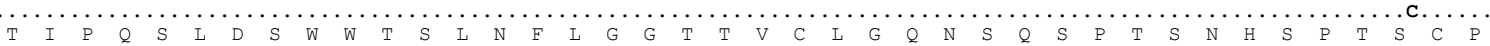

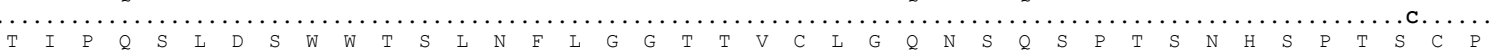

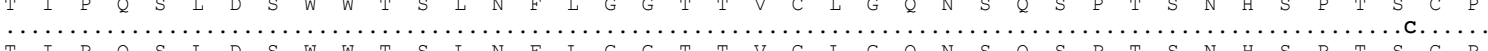

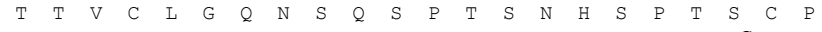

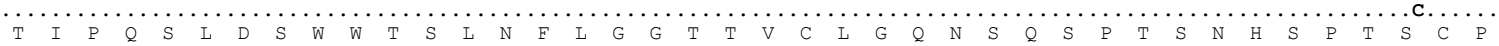
$T$
$T$

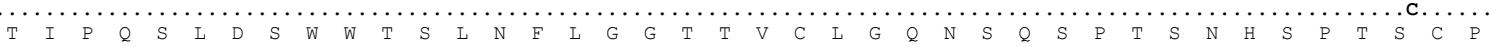

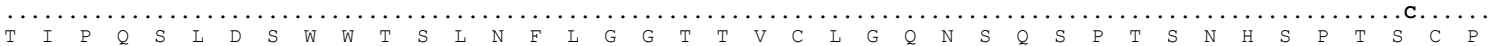

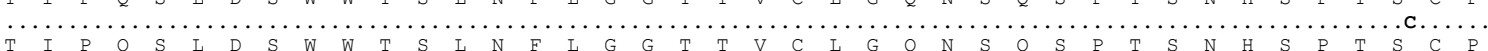

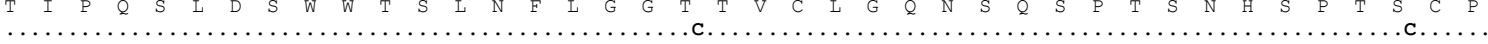

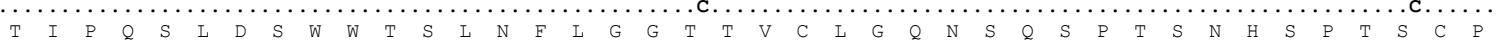

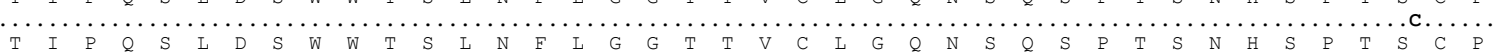

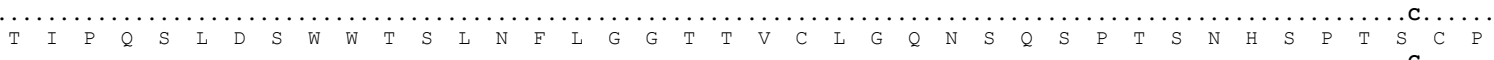

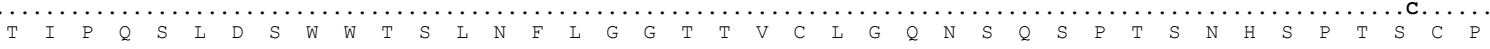

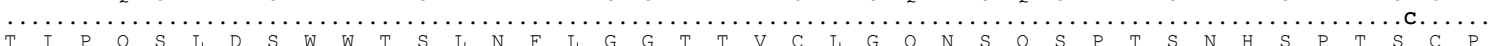

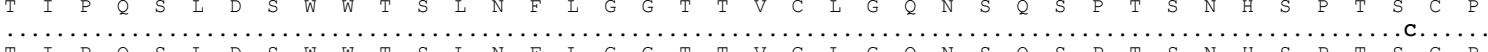

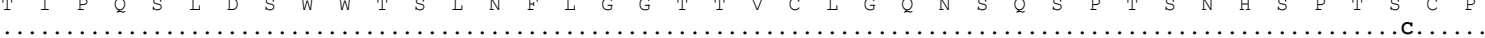

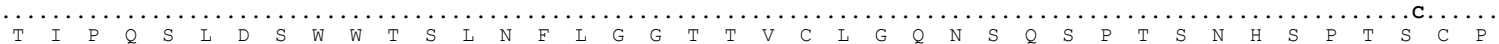

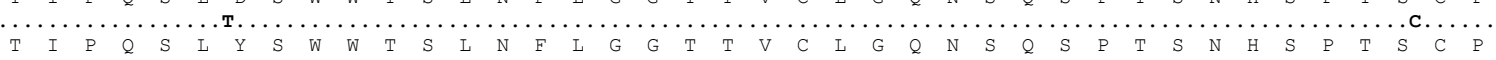

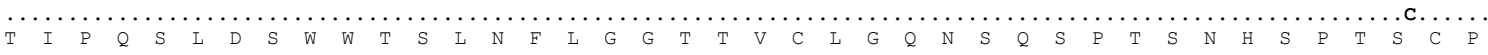

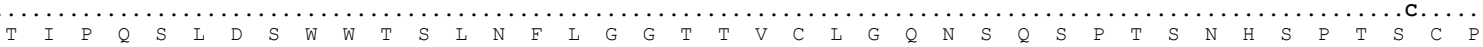

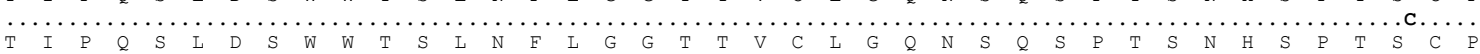

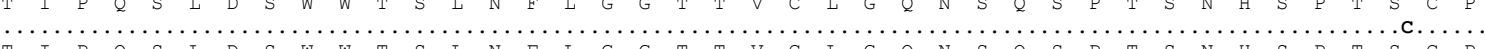

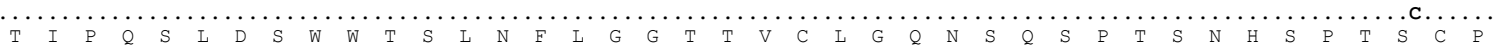

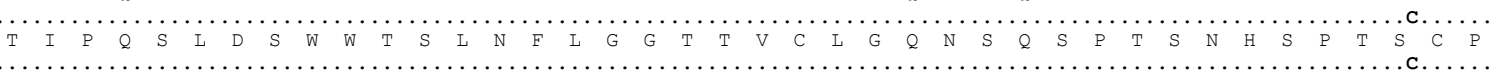

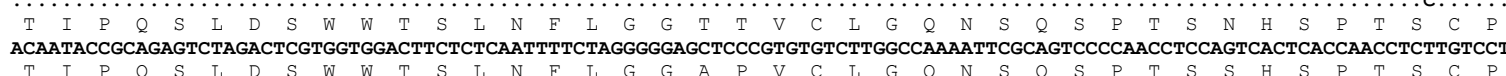
$T$
$T$ 
$\times 02763$

AH 1.II

AH 4 . I

AH 4-1835

AH 4-2015

AH 5-2878

AH 7-624

AH 7-640

AH 9-454

AH 7-1884

AH $10-2400$

AH 10-2596

CH 3-3608

CH 5-2628

CH $6-444 \mathrm{~A}$

CH 11-913

CH $12-82$

CH $12-2805$

COMPL 4-2971

COMPL 9-454

COMPL 9-1553

v01460

X02496

AH 2-2658

AH 3 . I

AH 3-577

AH 3-987

AH 3-7755

AH 3-7991

AH 4-775

AH 4-778

AH 4-2307

AH $4-3445$

AH 5.I

AH 5-556

AH 5-920

AH 5-1976

AH $6 . \mathrm{I}$

AH $6-217$

AH $6-220$

AH $7-283$ $\begin{array}{llllllllllll}360 & 370 & 380 & 390 & 400 & 410 & 420 & 430 & 440 & 450 & 460 & 470\end{array}$ CCAATTGTCCTGGTTATCGCTGGATGTGTCTGCGGCGTTTTATCATATTCCTCTTCATCCTGCTGCTATGCCTCATCTTCTTATTGGTTCTTCTGGATTATCAAGGTATGTTGCCCGTT

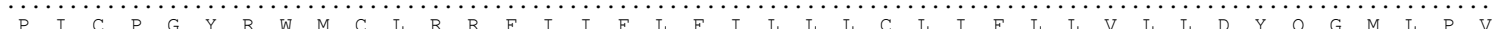

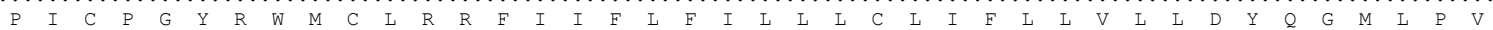

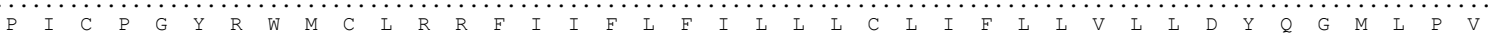

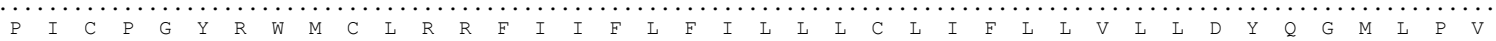

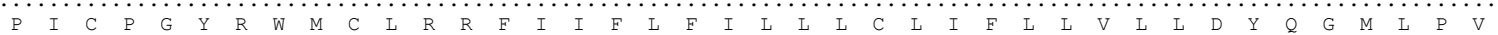

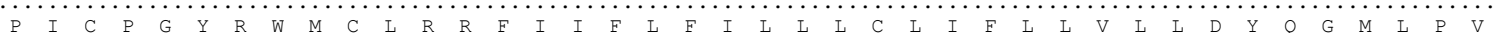

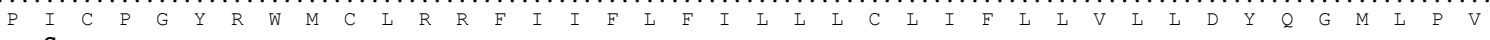

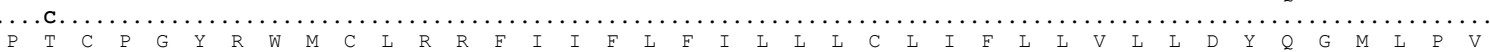

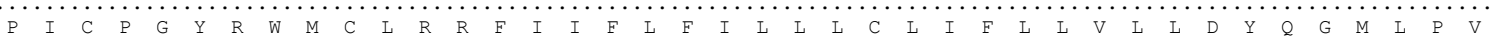
$P$
$P$

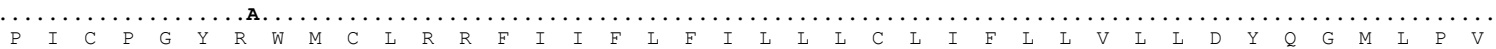

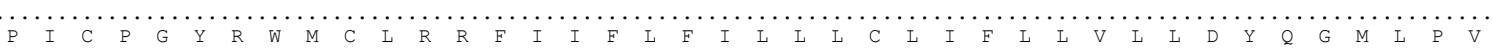

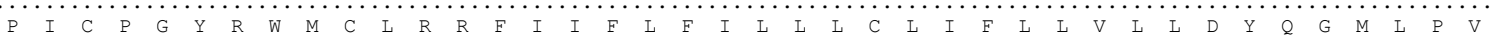

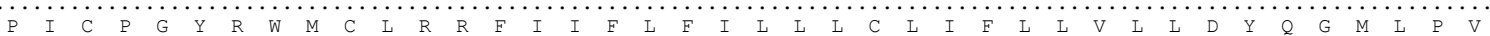

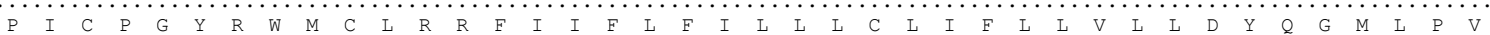

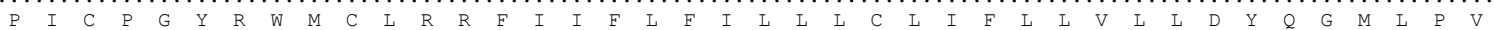

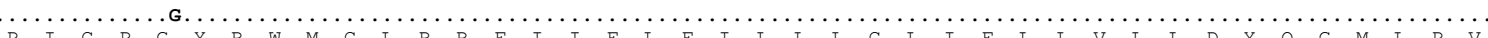

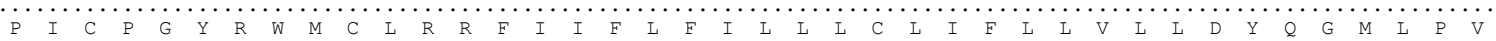

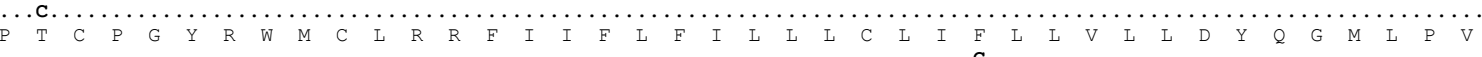

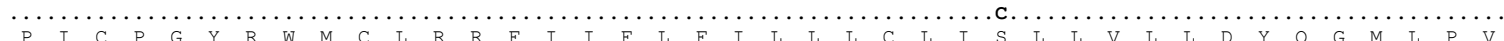
CCAACTTGTCCTGGTTATCGCTGGATGTGTCTGCGGCGTTTTATCATCTTCCTCTTCATCCTGCTGCTATGCCTCATCTTCTTGTTGGTTCTTCTGGACTATCAAGGTATGTTGCCCGTT

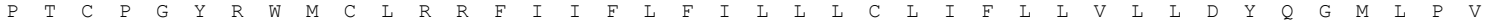

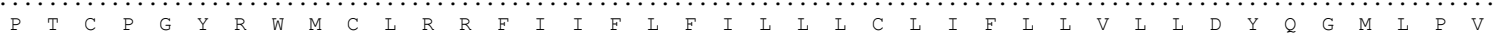

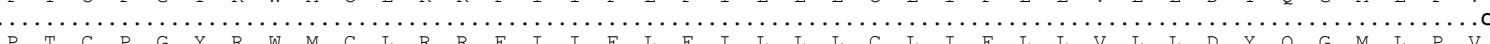

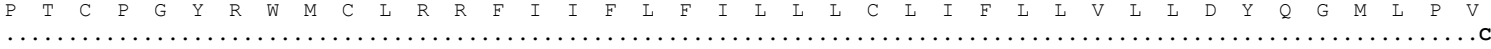

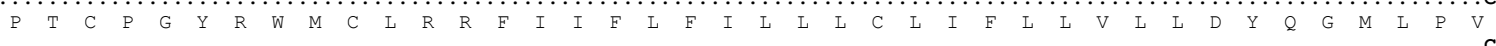

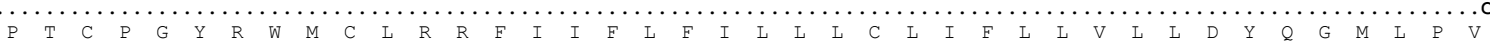

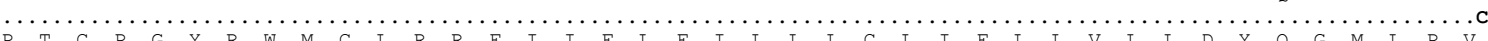

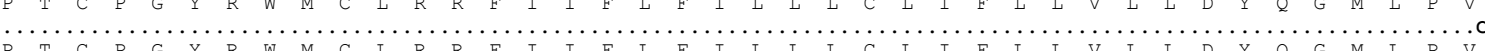

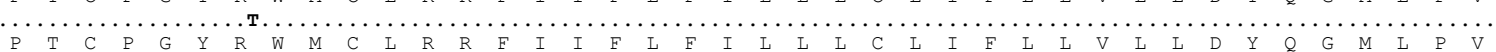

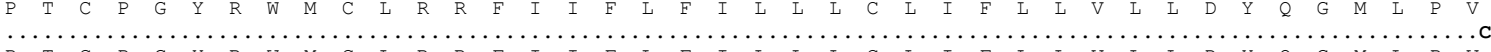
$P$
$P$

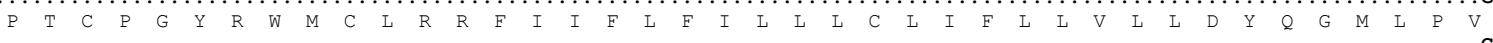
$P$
$P$

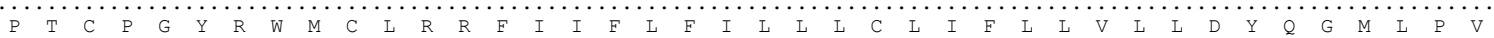

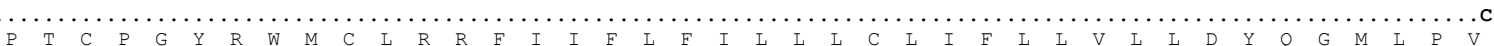

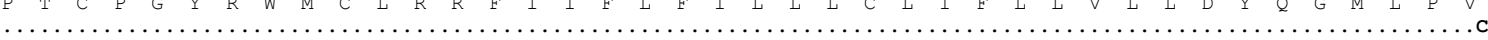

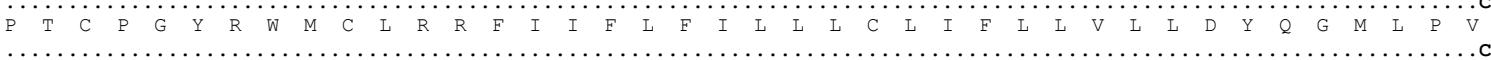

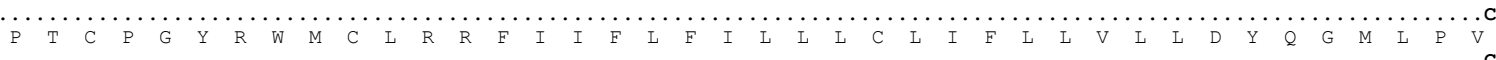

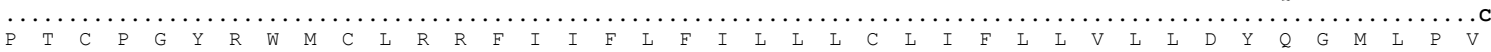

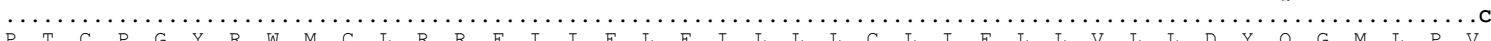

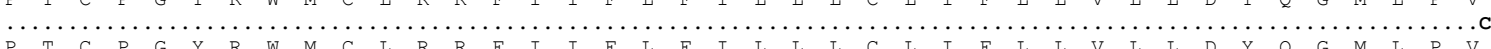

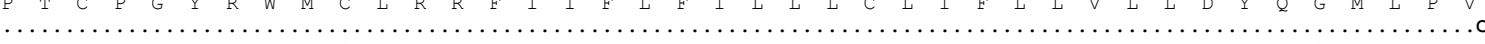

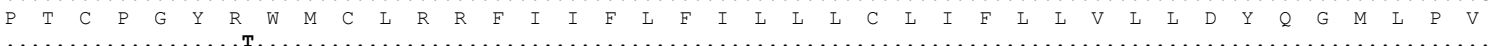

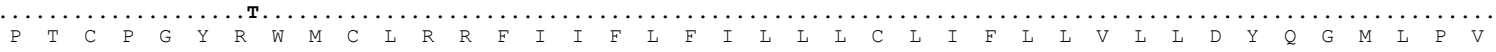


v01460

X02496

AH 7-496

AH 7-1010

AH 7-1275

AH 7-1442

AH $8-2186$

AH 9-2772

AH 9-296

AH 9-297

AH $9-3163$

AH 9-3113

AH 9-3897

AH 9-4070

AH $10-486$

AH $\quad$ 10-737

AH $10-885$

AH $10-1108$

AH $10-1612$

AH 10-2085

AH $10-2425$

AH 11-965

AH 11-1941

AH 11-2165

AH 11-2514

AH 11-2759

AH 12-116

CH 1-1546

CH 6-2107

CH 7-1117A

CH 9-599

CH 11-884

CH 11-2343

CH 12-958

COMPL 4-1725

COMPL 11-3028

COMPL 11-3179

X75657

AH 2-10101 $\begin{array}{lllllllllll}360 & 370 & 380 & 390 & 400 & 410 & 420 & 430 & 440 & 450 & 460\end{array}$

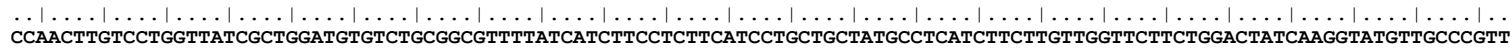
CCAACTTGTCCTGGTTATCGCTGGATGTGTCTGCGGCGTTTTATCATCTTCCTCTTCATCCTGCTGCTATGCCTCATCTTCTTGTTGGTTCTTCTGGACTATCAAGGTATGTTGCCCGTT

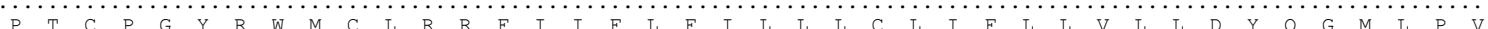

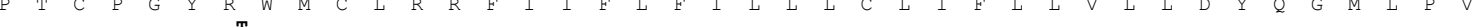

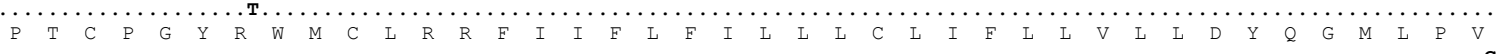

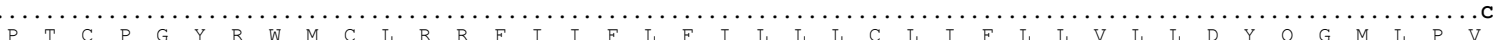

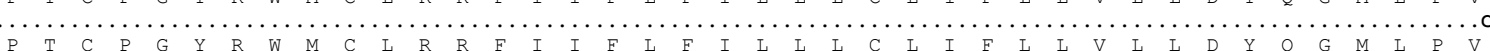

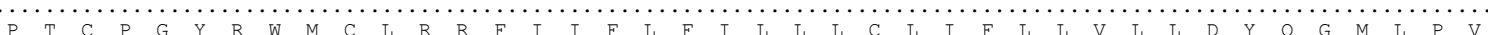

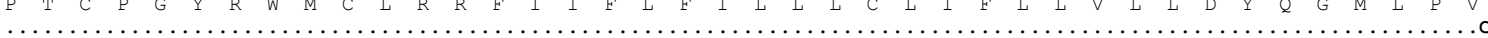

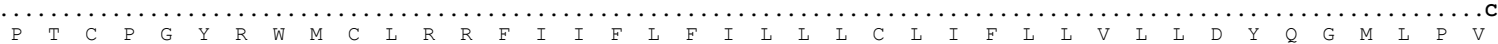

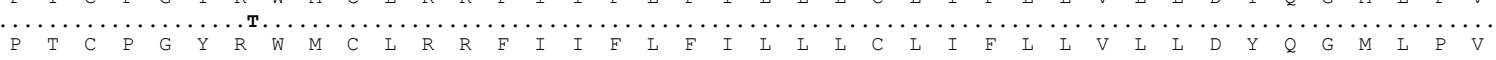

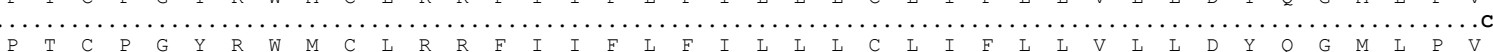

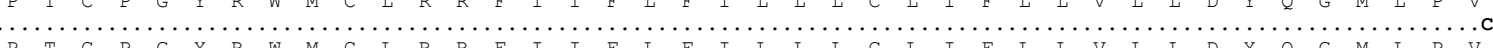

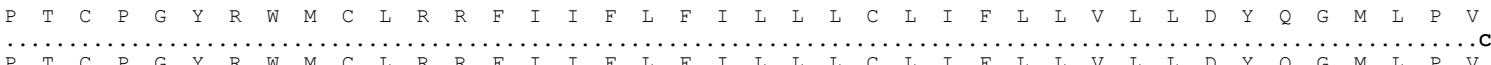

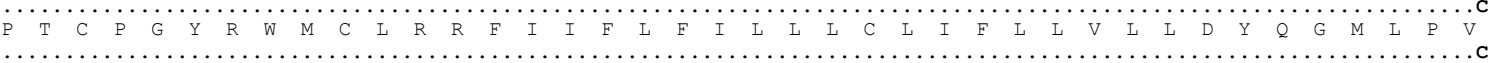

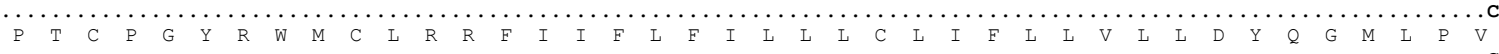

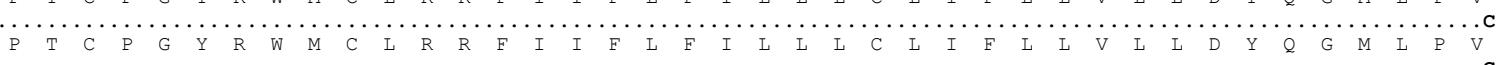

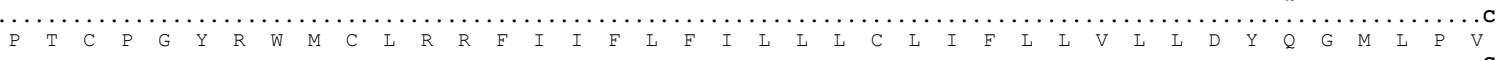

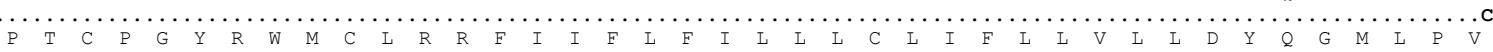

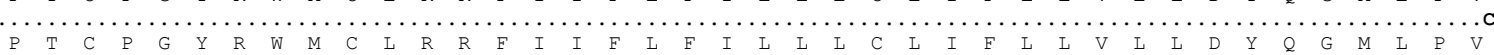

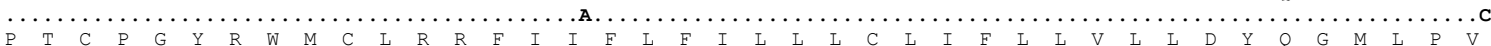

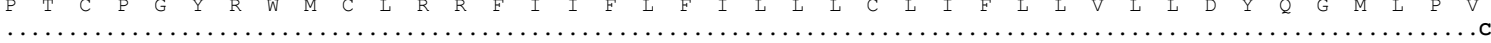

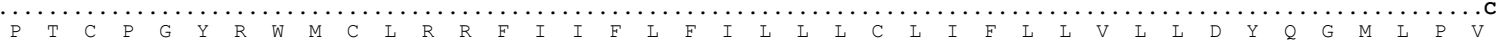

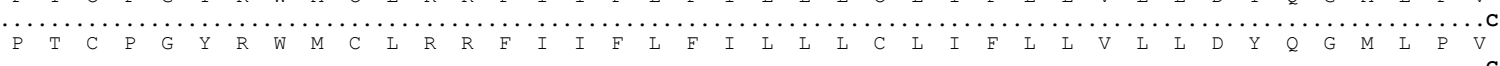

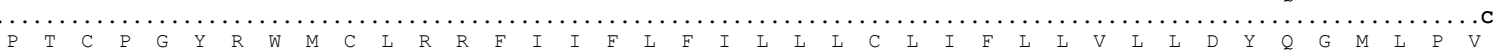

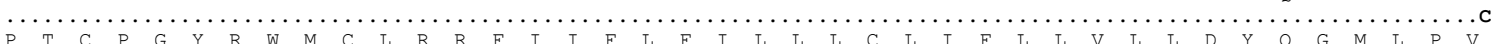

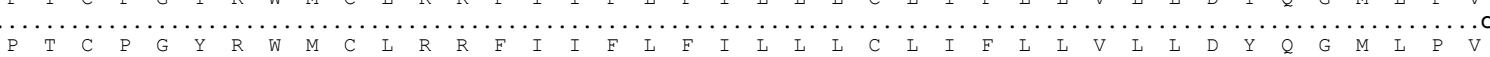

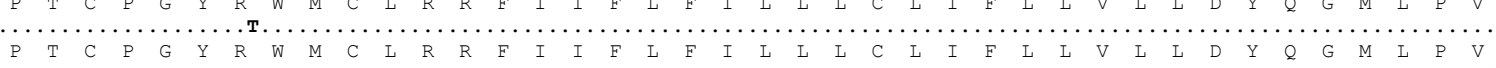

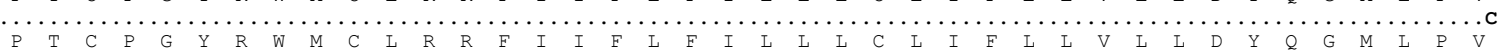

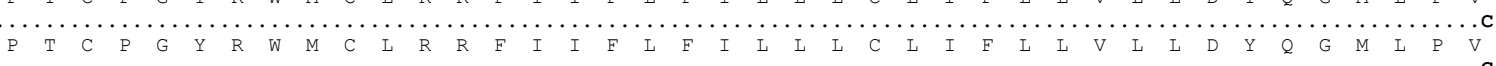
$P$
$P$

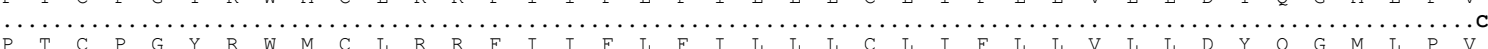

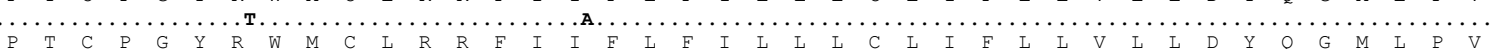

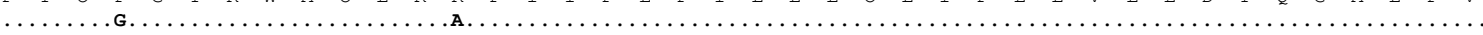

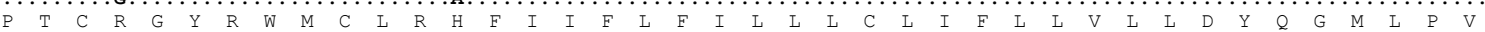

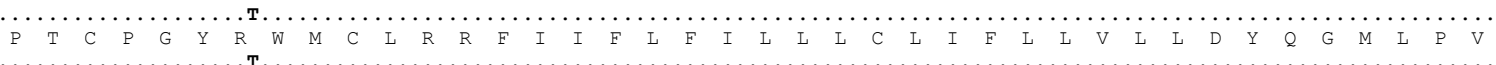

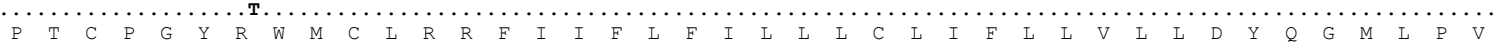

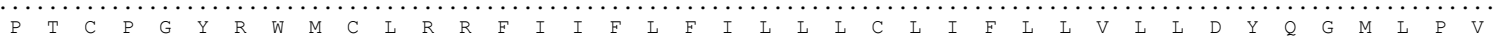

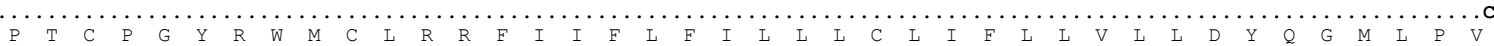

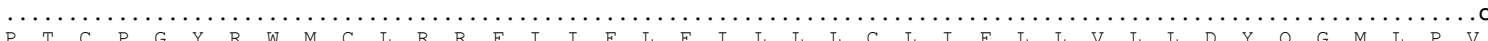

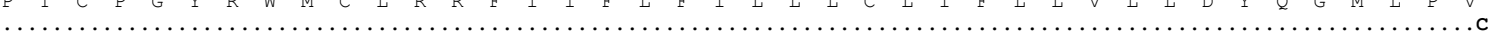

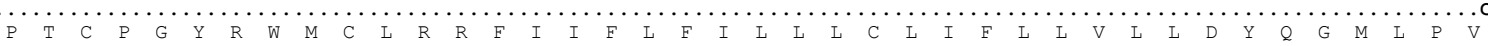

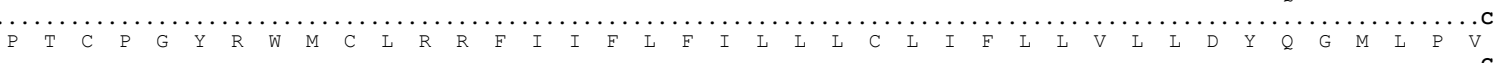
P
T PATTTTCCTGGCTATCGCTGGATGTGTCTGCGGCGTTTTATCATCTTCCTCTTCATCCTGCTGCTATGCCTCATCTTCTTGTTGGTTCTTCTGGACTATCAAGTATGTTGCCCGTI

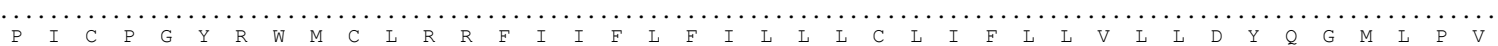


$x 02763$

AH 1.II

AH 4 . I

AH 4-1835

AH 4-2015

AH 5-2878

AH 7-624

AH 7-640

AH 9-454

AH 7-1884

AH $10-2400$

AH 10-2596

CH 3-3608

CH 5-2628

CH $6-444 \mathrm{~A}$

CH $11-913$

CH $12-82$

CH $12-2805$

COMPL 4-2971

COMPL 9-454

COMPL 9-1553

V01460

X02496

AH 2-2658

AH 3.I

AH 3-577

AH 3-987

AH 3-7755

AH 3-7991

AH 4-775

AH 4-778

AH 4-2307

AH $\quad 4-3445$

AH 5.I

AH 5-556

AH 5-920

AH 5-1976

AH 6 .I

AH $\quad 6-217$

AH $\quad 6-220$

AH 7-283

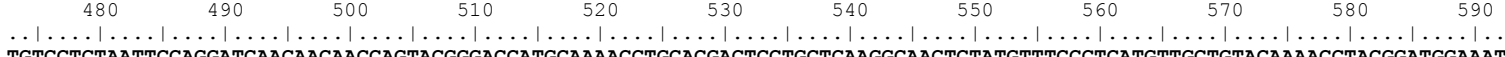
TGTCCTCTAATTCCAGGATCAACAACAACCAGTACGGGACCATGCAAAACCTGCACGACTCCTGCTCAAGGCAACTCTATGTTTCCCTCATGTTGCTGTACAAAACCTACGGATGGAAAT

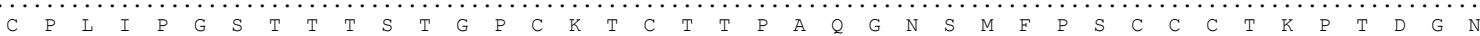

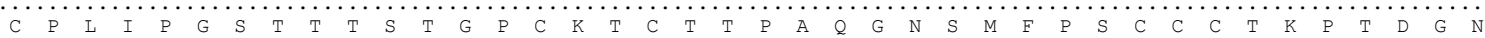

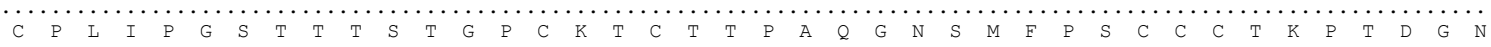

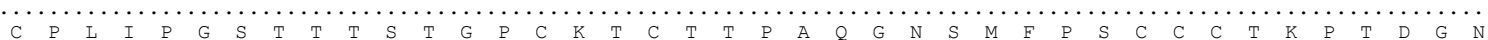

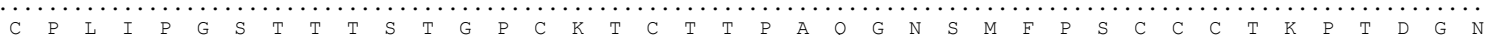

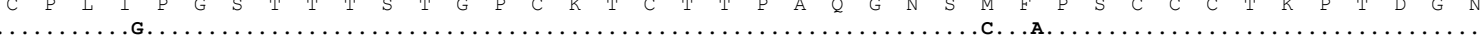
$C$
$C$ $C$
$C$

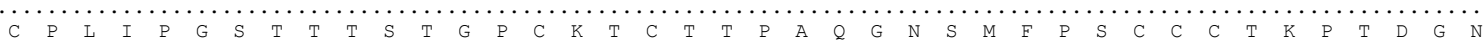

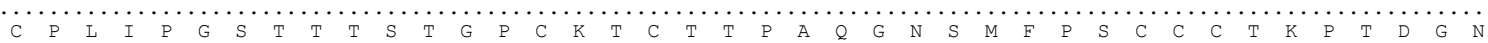

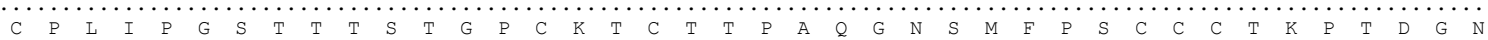

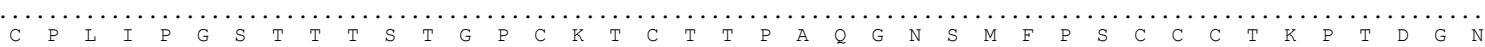

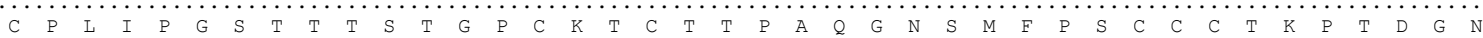

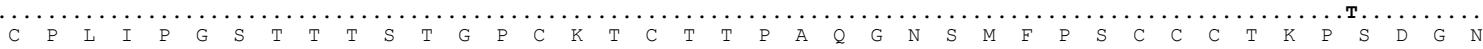
$C$
$C$

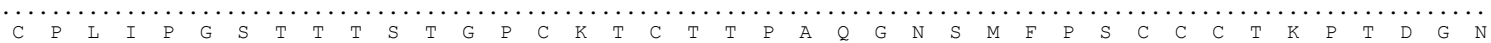
$C$
$C$

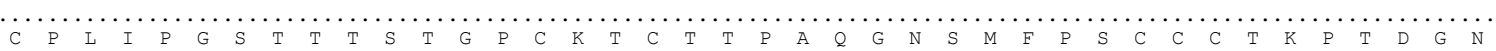

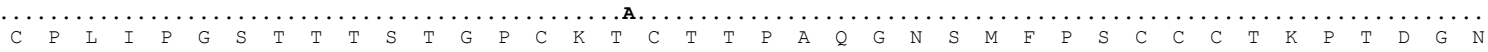

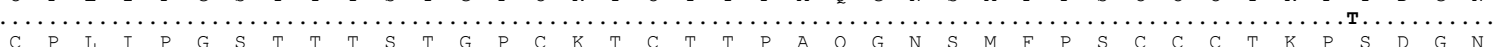
TGTCCTCTAATTCCAGGATCCTCAACAACCAGCACGGGACCATGCCGGACCTGCATGACTACTGCTCAAGGAACCTCTATGTATCCCTCCTGTTGCTGTACCAAACCTTCGGACGGAAAT

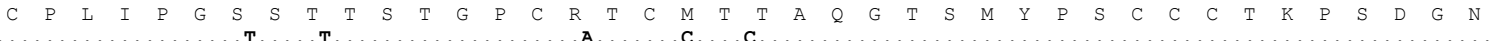
$C$
$C$

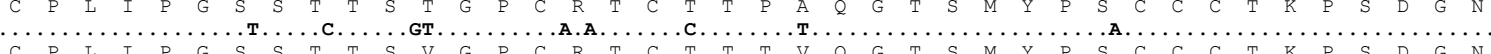

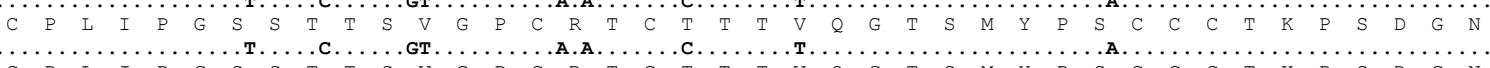
$C$
$C$

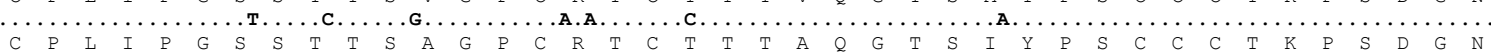

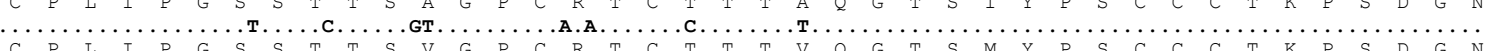
$\ldots \ldots \ldots$
$\ldots \ldots$ $C$
$C$

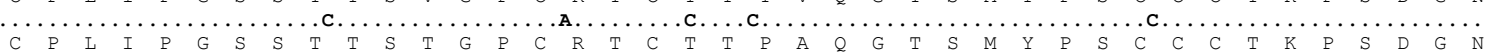

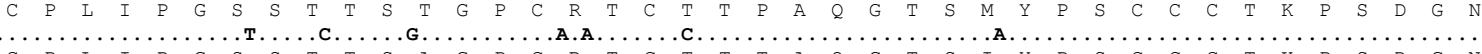

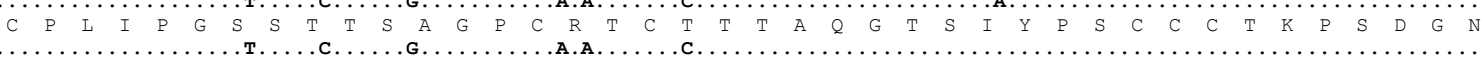
C $P$ P

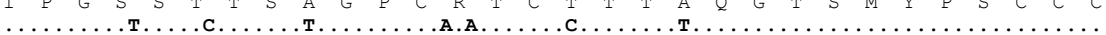

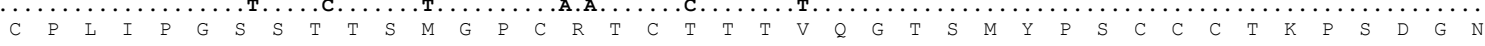

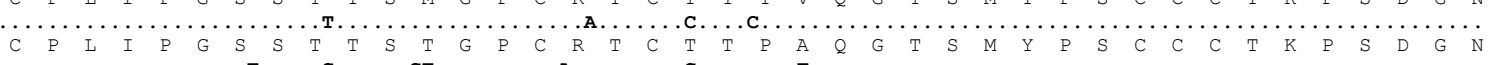

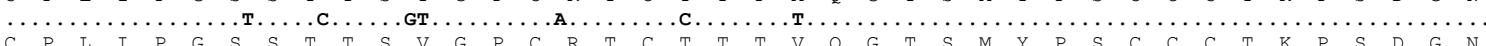

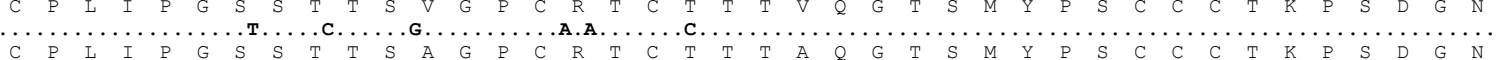

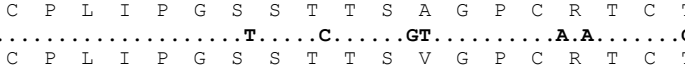
C $P$ L I P G S S T T S V G P C R T C T

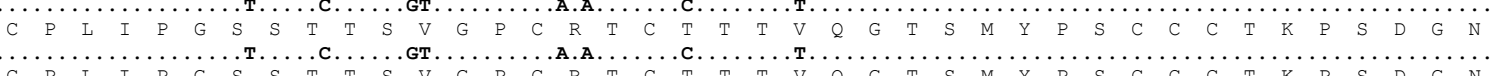

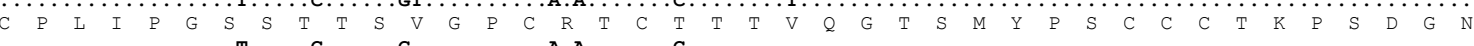

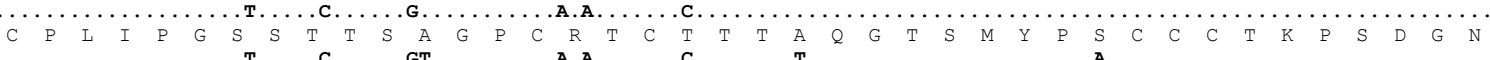

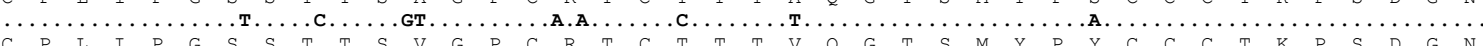

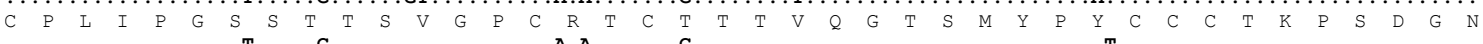

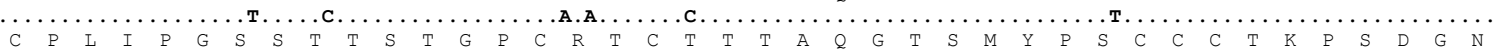


v01460

X02496

AH 7-496

AH 7-1010

AH 7-1275

AH 7-1442

AH $8-2186$

AH 9-2772

AH 9-296

AH 9-297

AH $9-3163$

AH 9-3113

AH 9-3897

AH 9-4070

AH $10-486$

AH 10-737

AH $10-885$

AH $\quad 10-1108$

AH 10-1612

AH 10-2085

AH $\quad$ 10-2425

AH 11-965

AH 11-1941

AH 11-2165

AH 11-2514

AH 11-2759

AH 12-116

CH 1-1546

CH 6-2107

CH 7-1117A

CH 9-599

CH 11-884

CH 11-2343A

CH 12-958

COMPL 4-1725

COMPL 11-3028

COMPL 11-3179

X75657

AH 2-10101

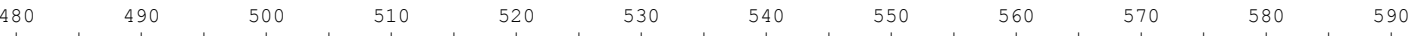
TGTCCTCTAATTCCAGGATCCTCAACAACCAGCACGGGACCATGCCGGACCTGCATGACTACTGCTCAAGGA.CCTCTATGTATCCCTCCTGTTGCTGTACCAAACCTTCGGACGGAAAT TGTCCTCTAATTCCAGGATCCTCAACAACCAGCACGGGACCATGCCGGACCTGCATGACTACTGCTCAAGGAACCTCTATGTATCCCTCCTGTTGCTGTACCAAACCTTCGGACGGAAA C...

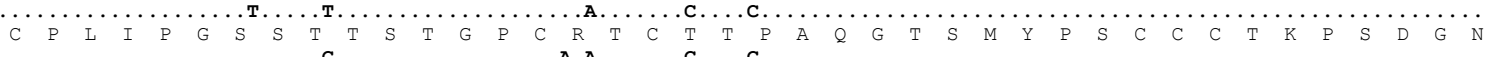

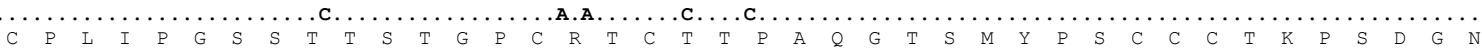

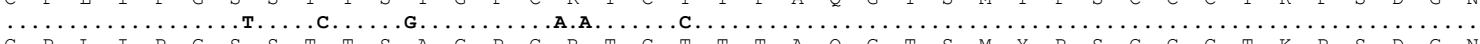

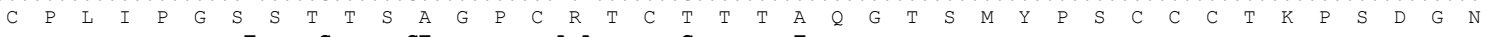

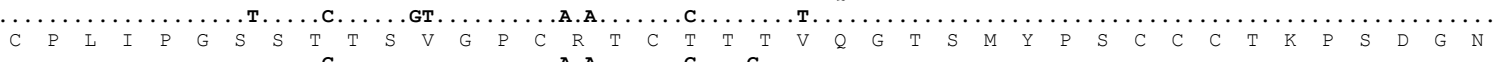

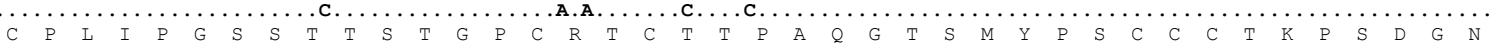

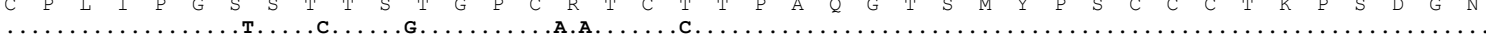

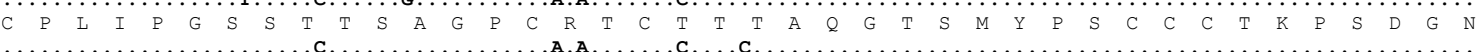
$\ldots \ldots$
$C$
$\ldots$

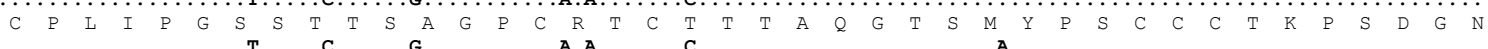
$C$
$C$

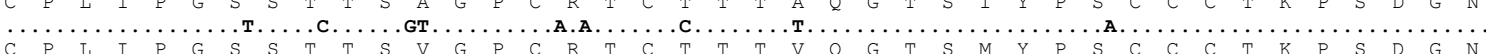

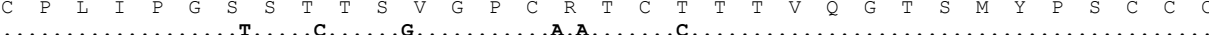
$C$
$P$ $\ldots$
$C$

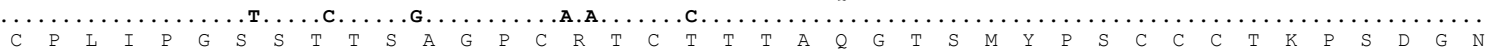

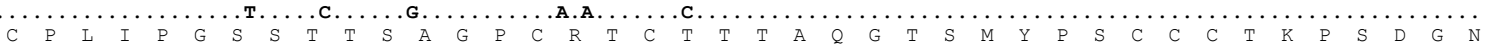

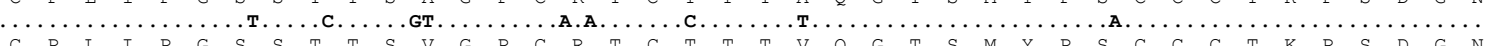

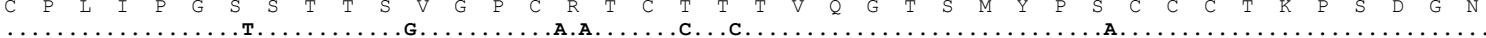

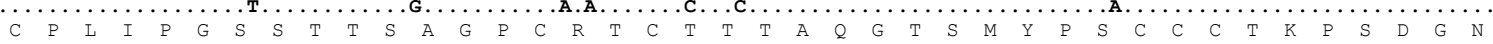

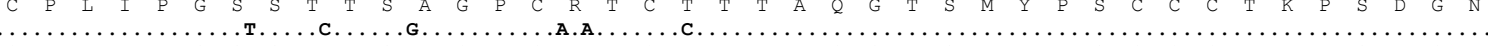

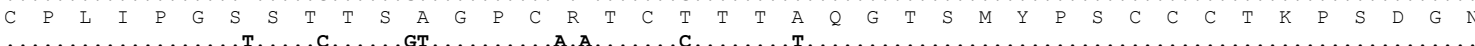

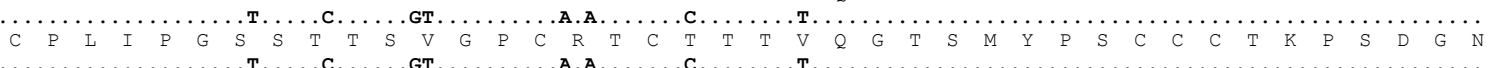
$C$
$C$

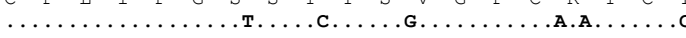
$C$
$C$

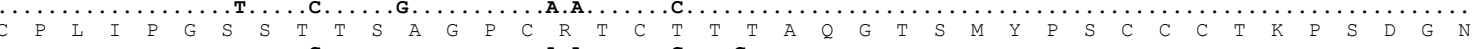

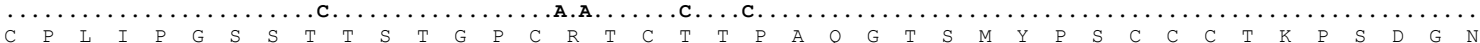

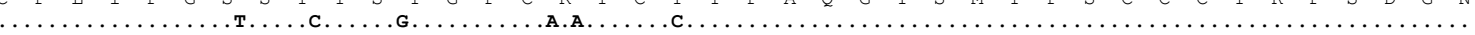

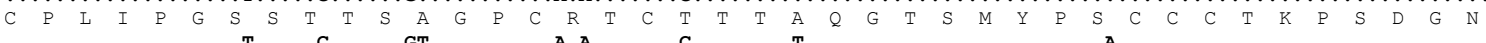
$C$
$C$

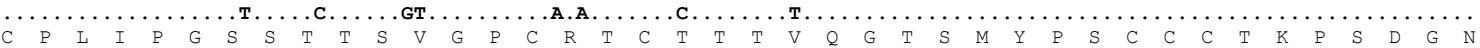

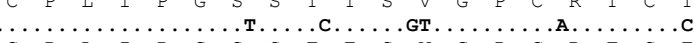

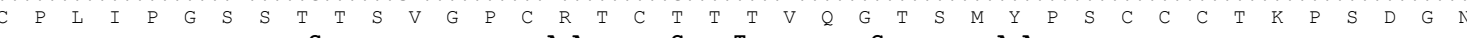

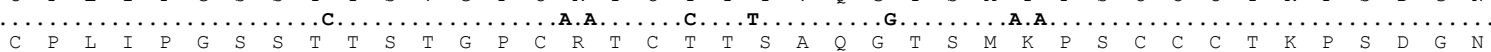

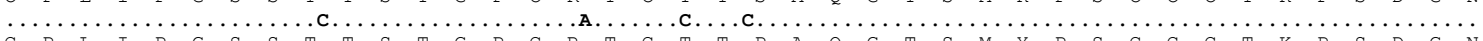
$\begin{array}{llllllllllllllllllllllllll}C & P & L & I & P & G & S & S & T & T & S & T & G & P & C & R & T & C & T & T & P & A & Q & G & \end{array}$

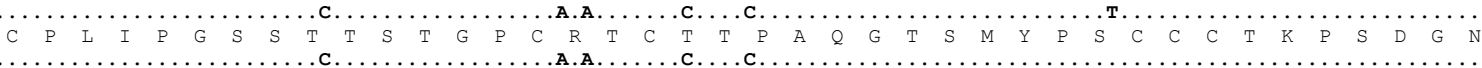

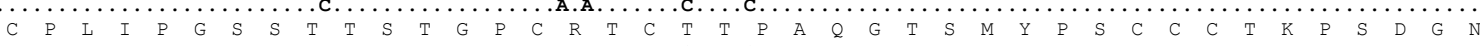

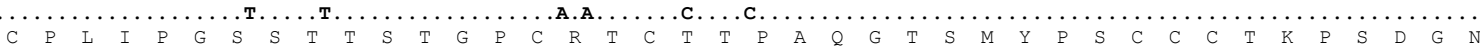

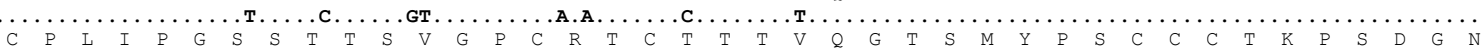

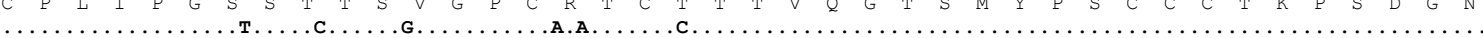
$C$
$C$

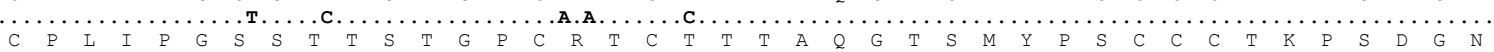

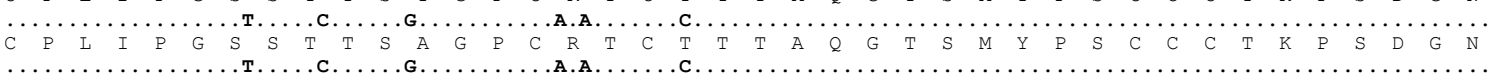

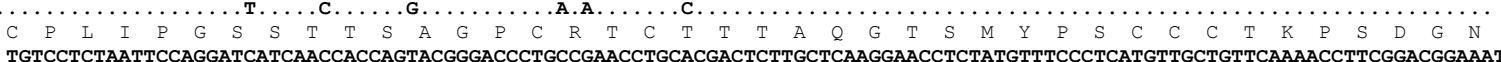

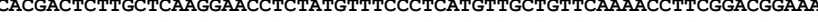

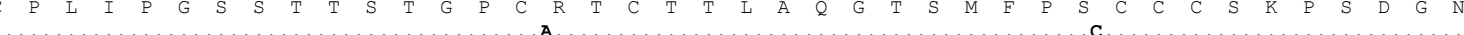

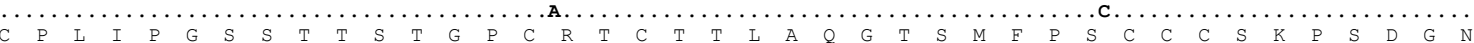


$\mathrm{x} 02763$

AH 1.II

AH 4 . I

AH 4-1835

AH 4-2015

AH 5-2878

AH 7-624

AH 7-640

AH 9-454

AH 7-1884

AH $10-2400$

AH 10-2596

CH 3-3608

CH 5-2628

CH $6-444 \mathrm{~A}$

CH 11-913

CH $12-82$

CH 12-2805

COMPL 4-2971

COMPL 9-454

COMPL 9-1553

V01460

X02496

AH 2-2658

AH 3 . I

AH 3-577

AH 3-987

AH 3-7755

AH 3-7991

AH 4-775

AH 4-778

AH 4-2307

AH $\quad 4-3445$

AH 5.I

AH 5-556

AH 5-920

AH 5-1976

AH $6 . \mathrm{I}$

AH $6-217$

AH $6-220$

AH 7-283

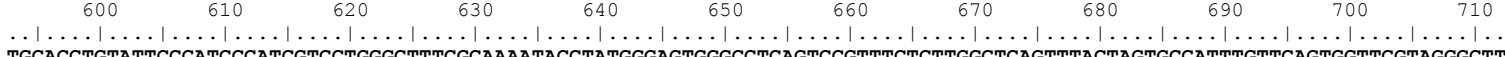
TGCACCTGTATTCCCATCCCATCGTCCTGGGCTTTCGCAAAATACCTATGGGAGTGGGCCTCAGTCCGTTTCTCTTGGCTCAGTTTACTAGTGCCATTTGTTCAGTGGTTCGTAGGGCTT

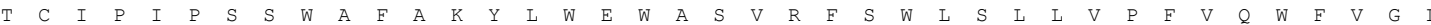

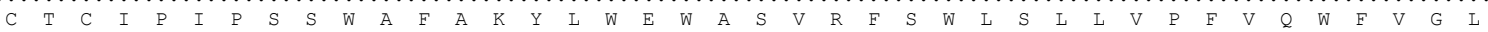

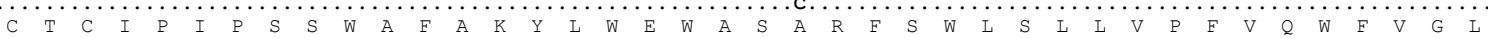

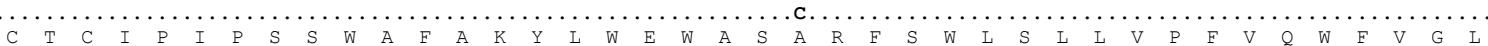
C .

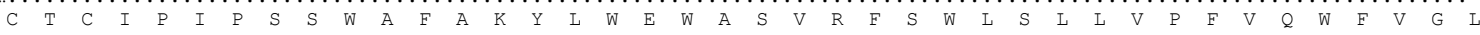

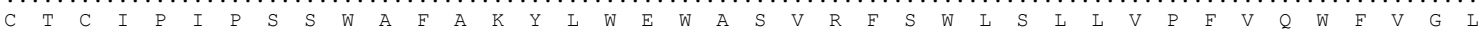

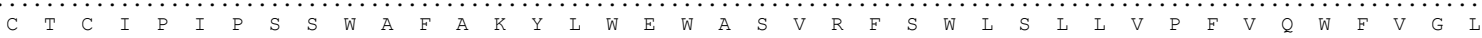

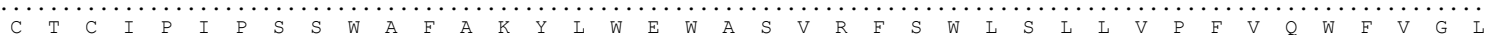

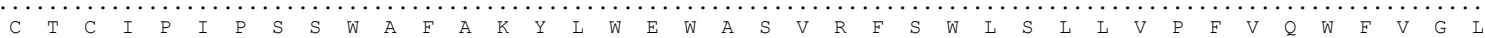

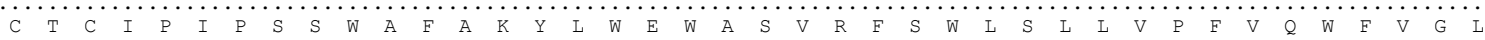

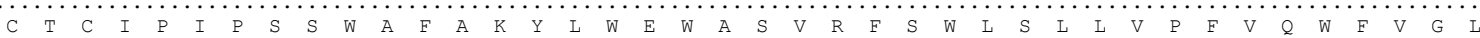
$C$
$C$

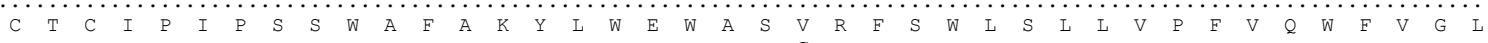

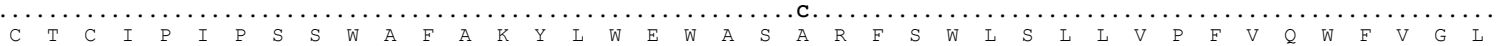

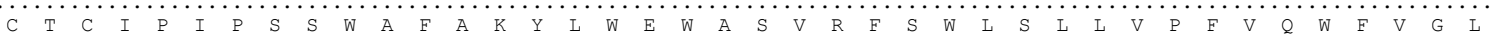

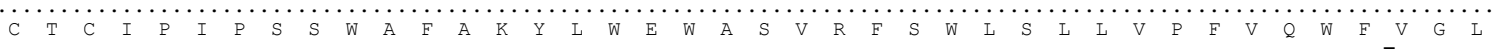

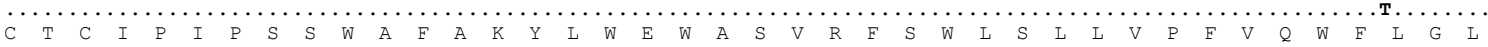

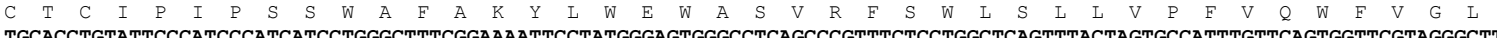

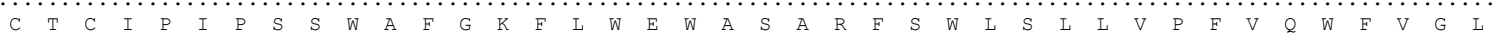

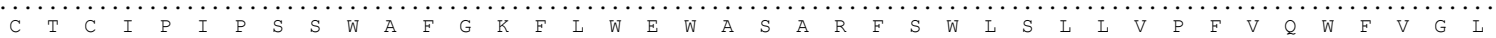

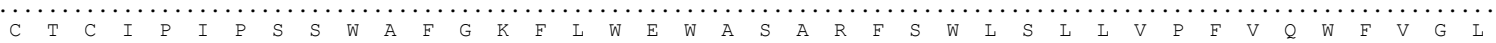

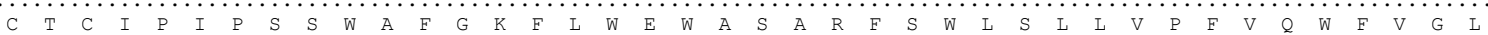

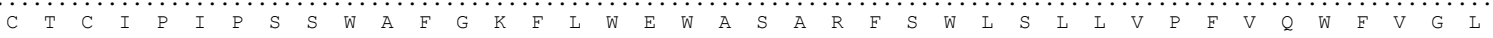

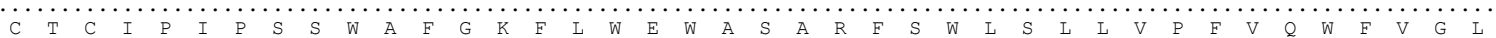

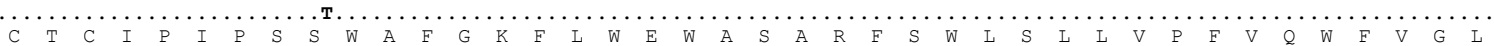

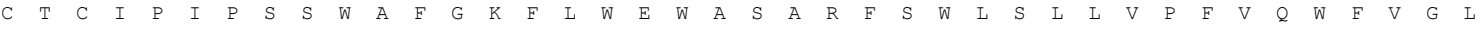

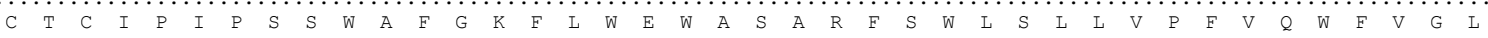

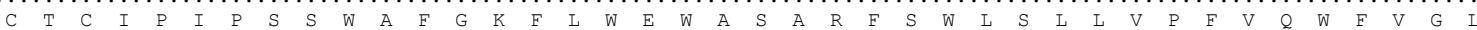
$C$
$C$ $C$
$T$

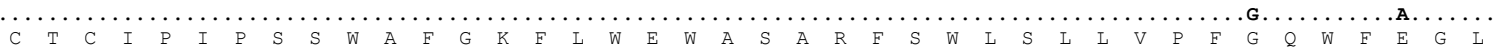

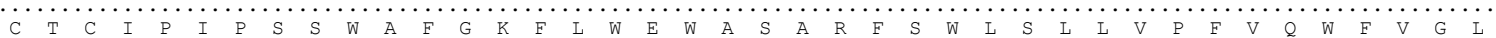

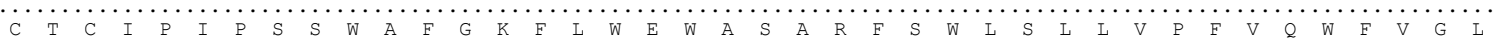

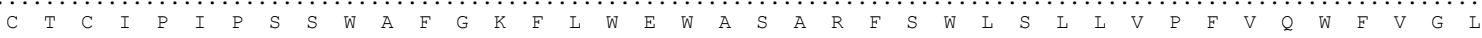

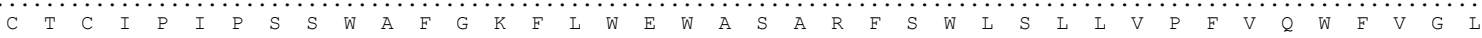

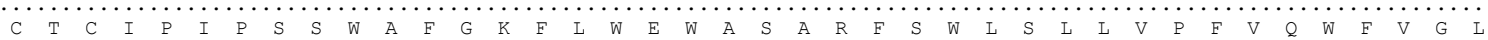

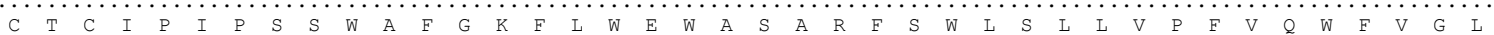

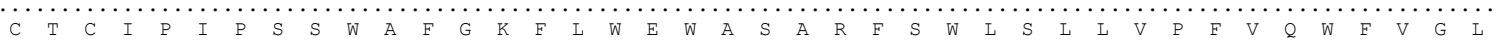


v01460

$\mathrm{x} 02496$

AH 7-496

AH 7-1010

AH 7-1275

AH 7-1442

AH $8-2186$

AH 9-2772

AH 9-296

AH 9-297

AH $9-3163$

AH 9-3113

AH 9-3897

AH $9-4070$

AH $10-486$

AH 10-737

AH $10-885$

AH $10-1108$

AH 10-1612

AH 10-2085

AH $10-2425$

AH 11-965

AH 11-1941

AH $11-2165$

AH 11-2514

AH 11-2759

AH 12-116

CH 1-1546

CH 6-2107

CH 7-1117A

CH 9-599

CH 11-884

CH 11-2343A

CH 12-958

COMPL 4-1725

COMPL 11-3028

COMPL 11-3179

X75657

AH 2-10101

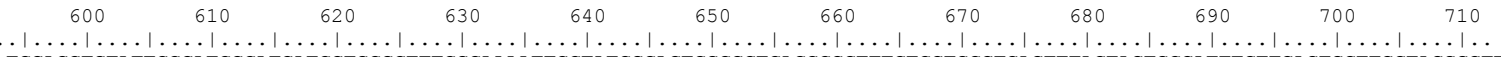
TGCACCTGTATTCCCATCCCATCATCCTGGGCTTTCGGAAAATTCCTATGGGAGTGGGCCTCAGCCCGTTTCTCCTGGCTCAGTTTACTAGTGCCATTTGTTCAGTGGTTCGTAGGGCTI

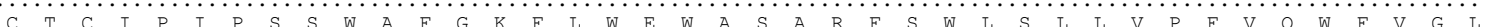

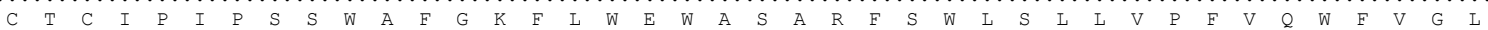
C $T$ C I P I P S S W A F G K F L W E W A S A R F S W L S I L V P F V O W F V G L

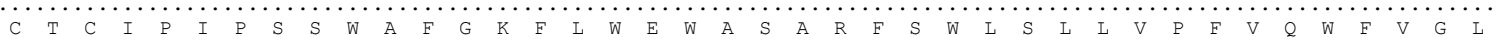

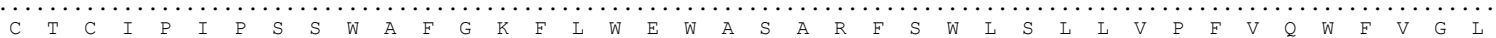

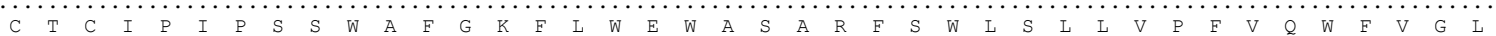

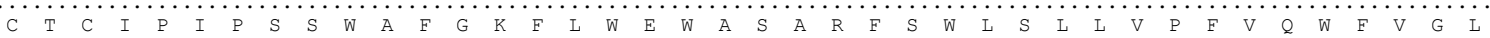

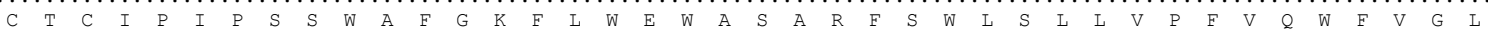

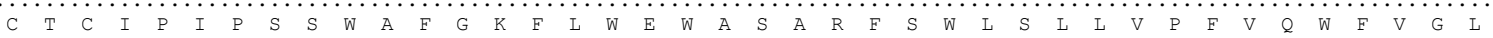

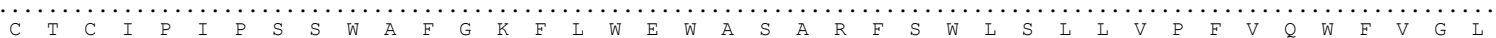

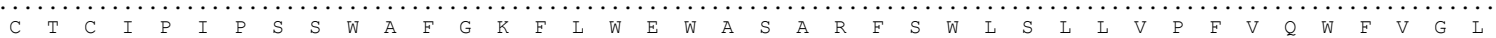

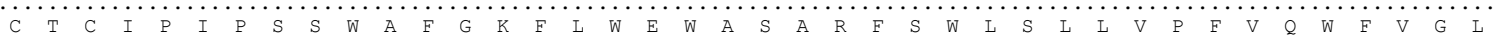

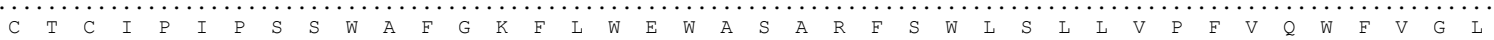

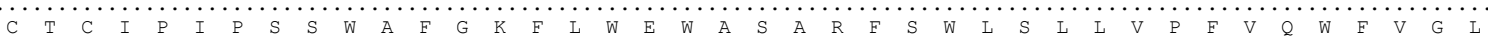

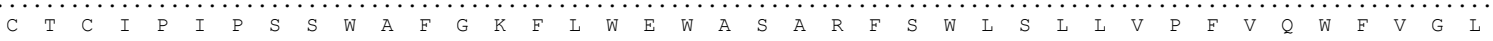

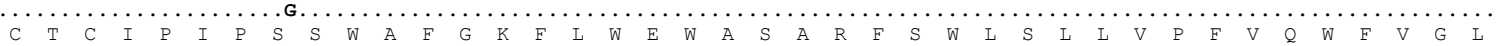

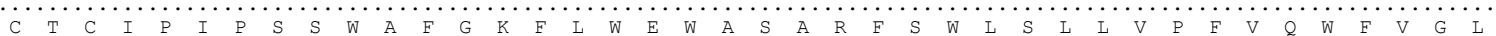

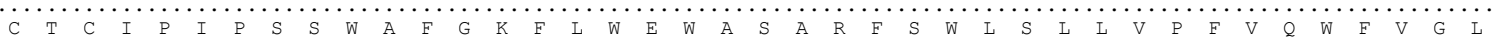

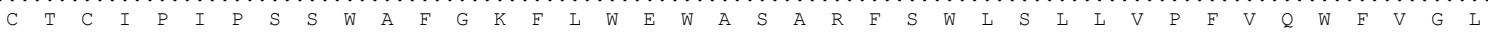

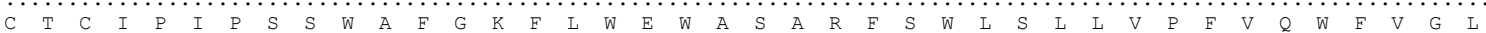

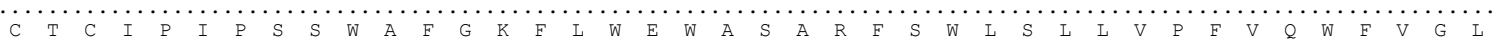

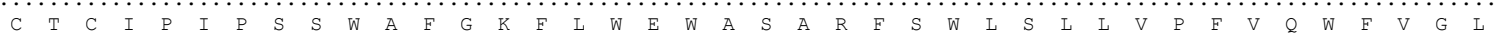

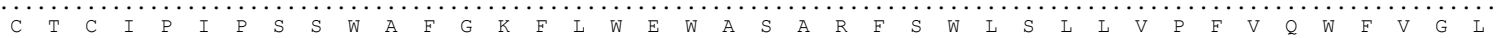

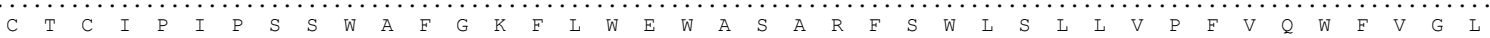

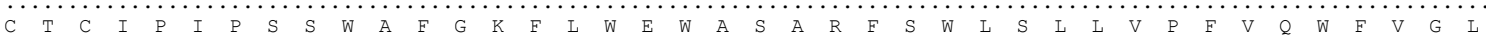

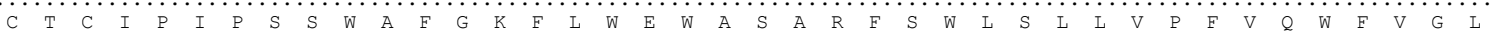

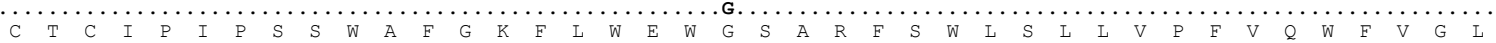

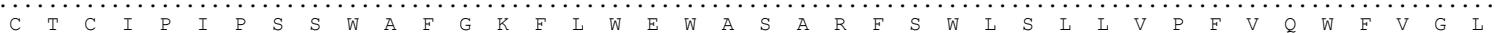

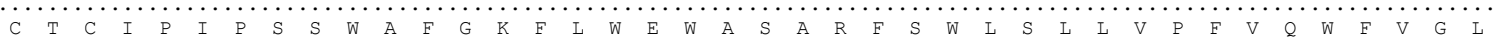

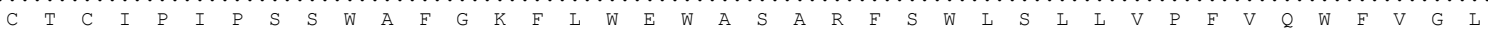

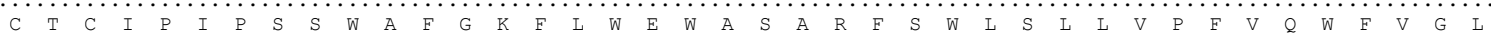

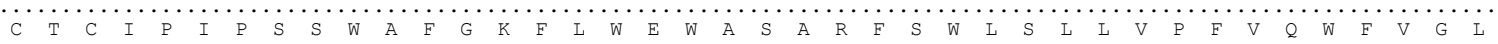

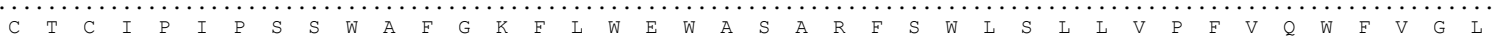

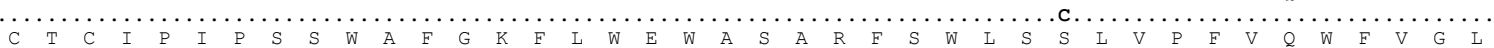

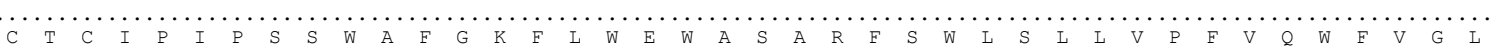

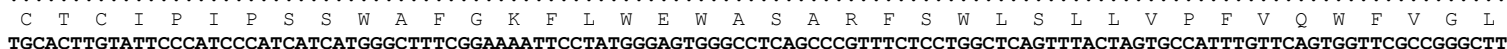

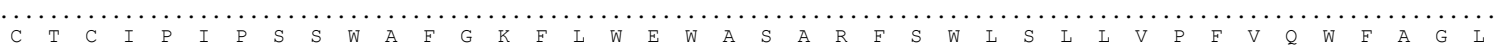


$\mathrm{X} 02763$

AH 1. II

AH 4 . I

AH 4-1835

AH 4-2015

AH 5-2878

AH 7-624

AH $7-640$

AH $9-454$

AH 7-1884

AH $10-2400$

AH 10-2596

CH 3-3608

CH 5-2628

CH $6-444 \mathrm{~A}$

CH 11-913

CH 12-82

CH 12-2805

COMPL 4-2971

COMPL 9-454

COMPL 9-1553

v01460

X02496

AH 2-2658

AH 3.I

AH 3-577

AH $3-987$

AH 3-7755

AH 3-7991

AH 4-775

AH 4-778

AH 4-2307

AH 4-3445

AH 5.I

AH 5-556

AH 5-920

AH 5-1976

AH 6.I

AH 6-217

AH $6-220$

AH 7-283

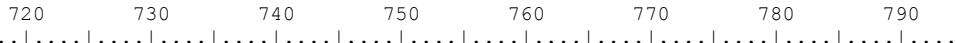
TCCCCCACTGTTTGGCTTTCAGCTATATGGATGATGTGGTATTGGGGCCAAGTCTGTACAGCATCGTGAGTCCCTTTATACC

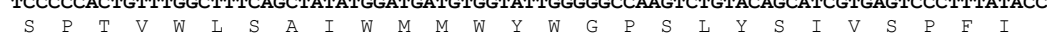

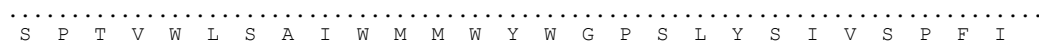

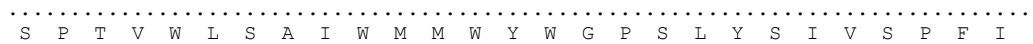

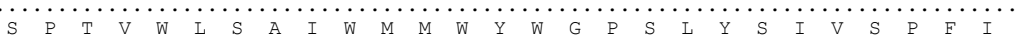

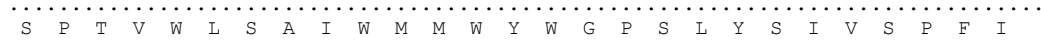

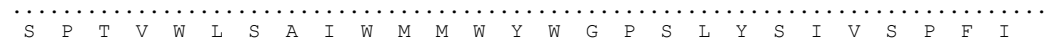

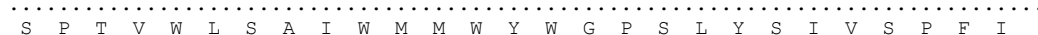

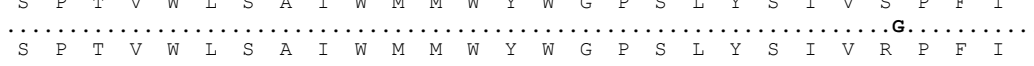

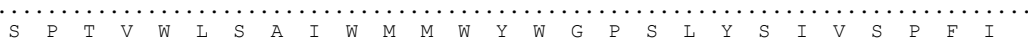

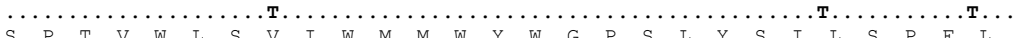
n.m. $\begin{array}{llllllllllllllllllllllllllllllllll} & P & T & V & W & L & S & A & I & W & M & M & W & Y & W & G & P & S & L & Y & S & I & V & S & P & F & I\end{array}$

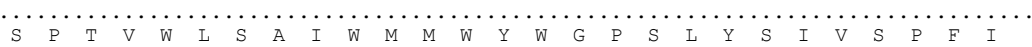

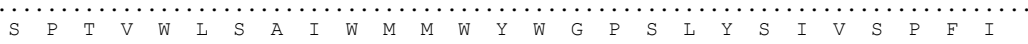

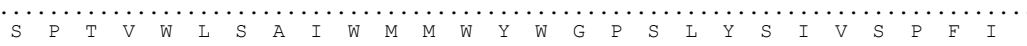

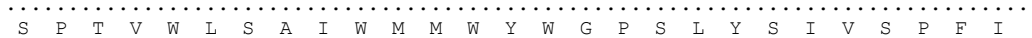

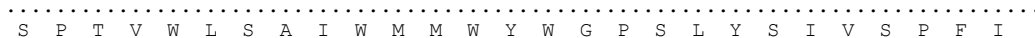

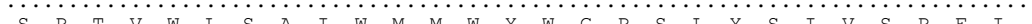

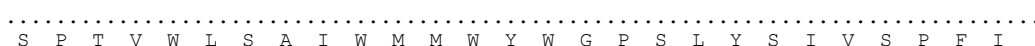
$\begin{array}{lllllllllllllllllllllllllllllllll}S & P & T & V & W & L & S & A & I & W & M & M & W & Y & W & G & P & S & L & Y & S & I & V & S & P & F & I\end{array}$

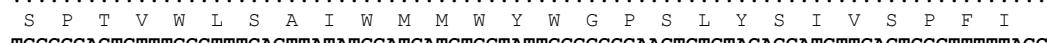

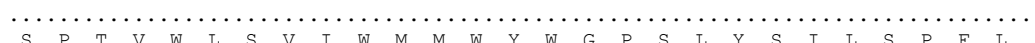

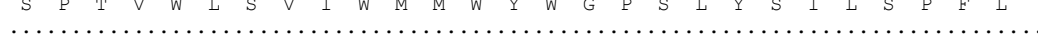
$M$
$S$ \begin{tabular}{l}
$M$ \\
\hline
\end{tabular}

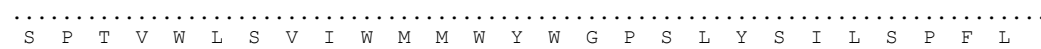

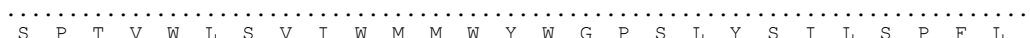

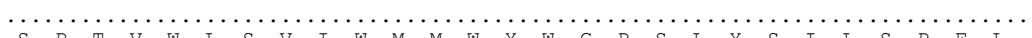

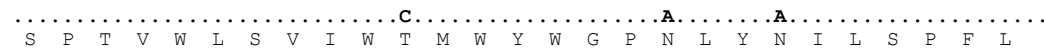

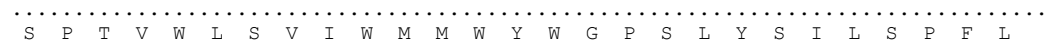

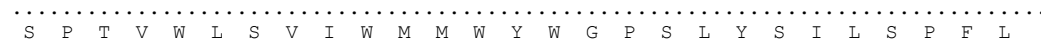

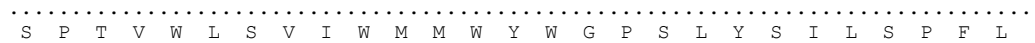

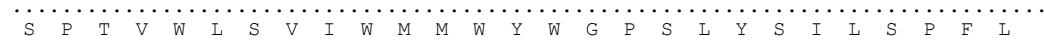

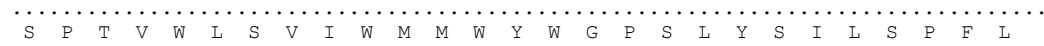

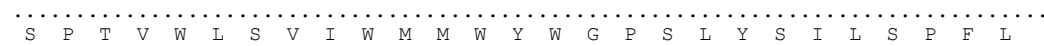
$M$
$S$

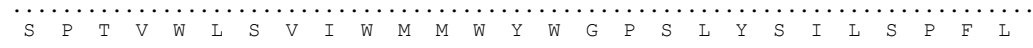

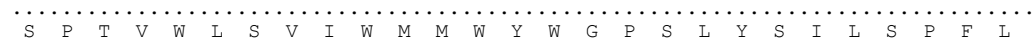

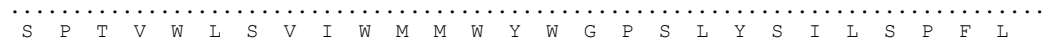

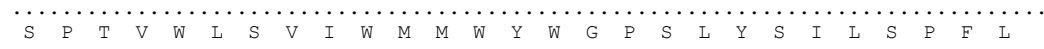

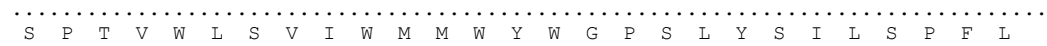




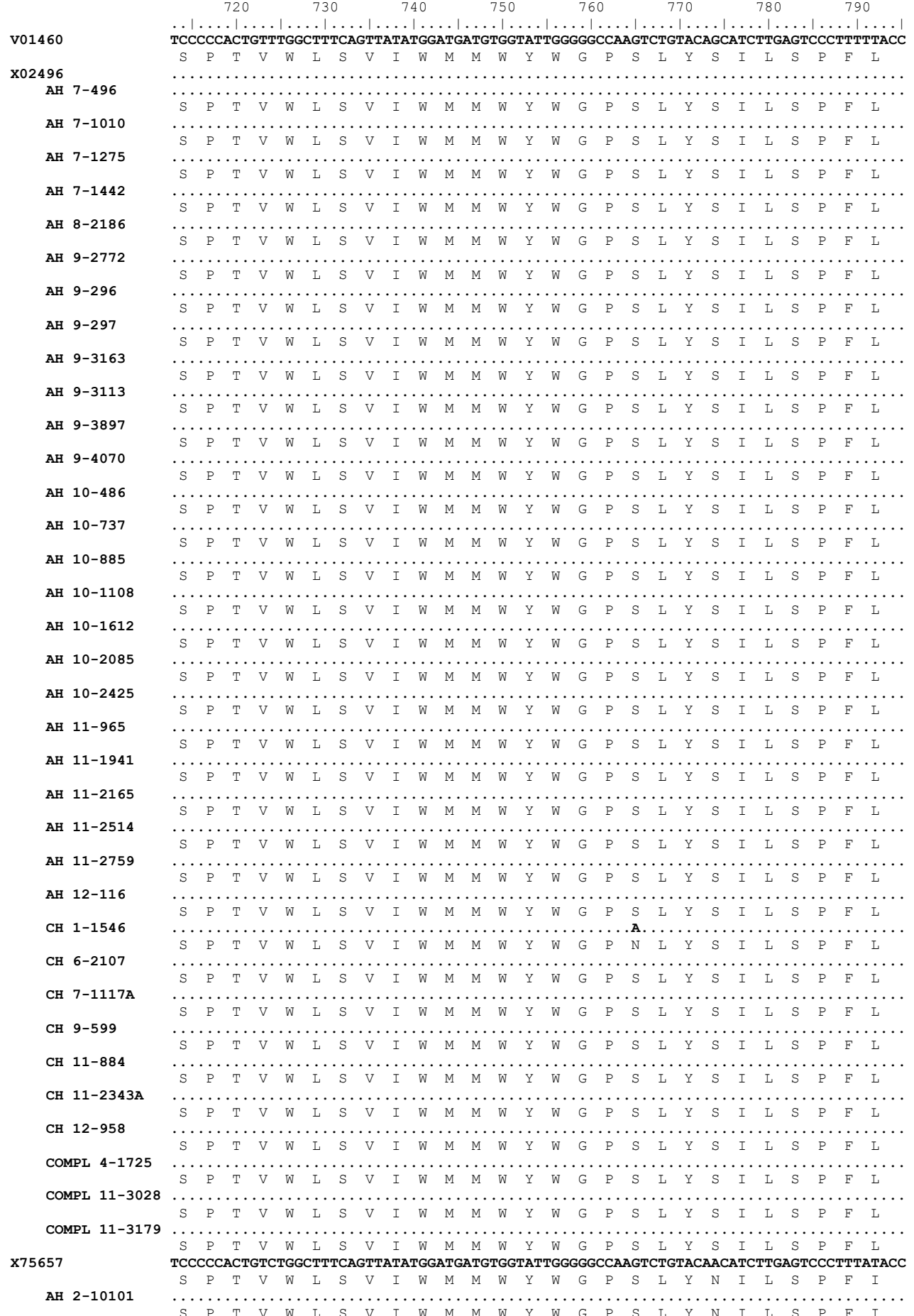


Appendix Part C. Sequences of gene X obtained during the study.

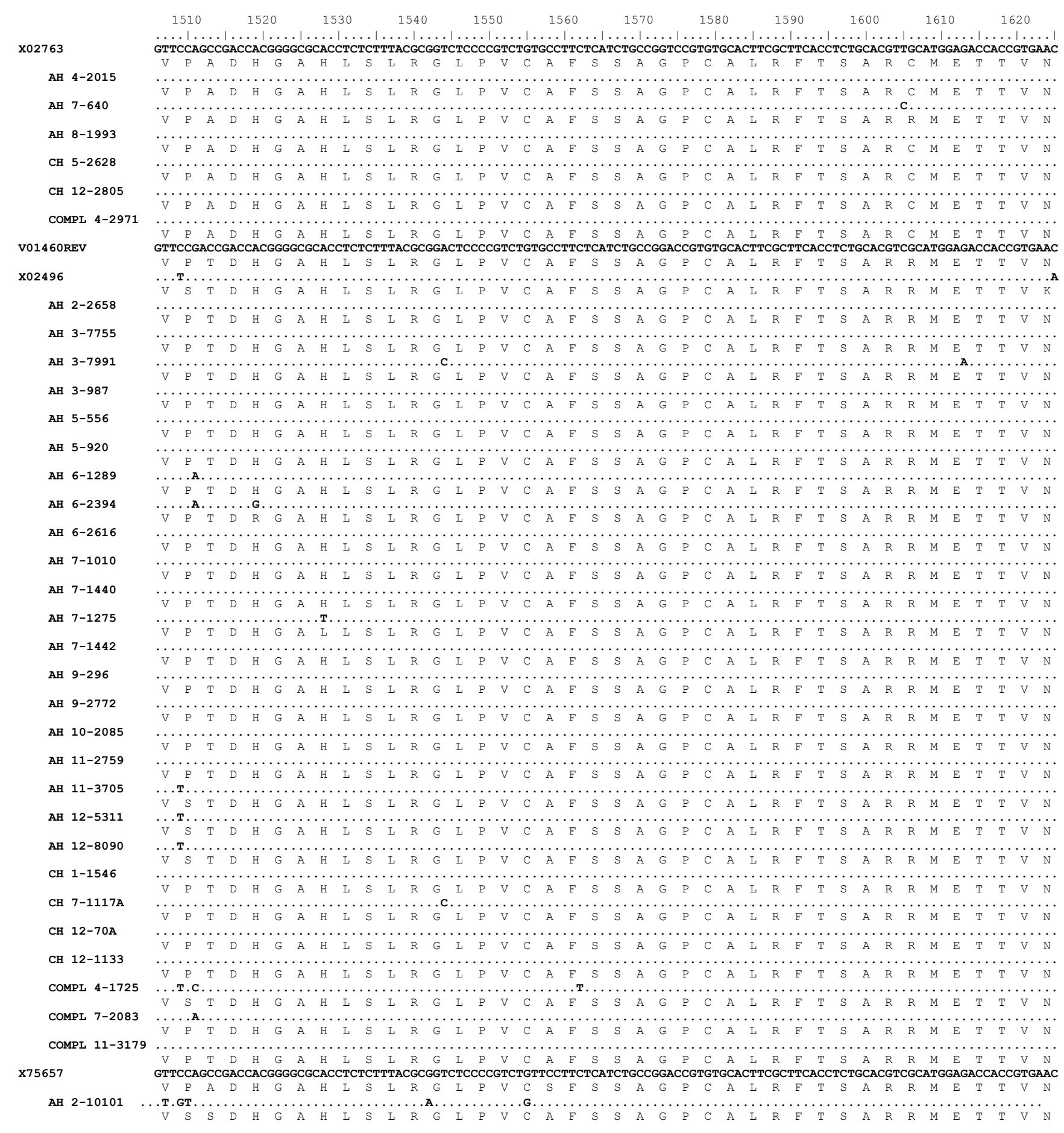


X02763

AH 4-2015

AH 7-640

AH $8-1993$

CH 5-2628

CH 12-2805

COMPL 4-2971

V01460REV

$\mathrm{X} 02496$

AH 2-2658

AH 3-7755

AH 3-7991

AH 3-987

AH 5-556

AH $5-920$

AH 6-1289

AH $\quad$ 6-2394

AH $6-2616$

AH $7-1010$

AH 7-1440

AH $7-1275$

AH 7-1442

AH 9-296

AH 9-2772

AH 10-2085

AH 11-2759

AH 11-3705

AH 12-5311

AH $12-8090$

CH 1-1546

CH 7-1117A

CH 12-70A

CH $12-1133$

COMPL 4-1725

COMPL 7-2083

COMPL 11-3179

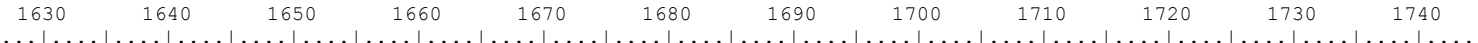
GCCCATCAGATCCTGCCCAAGGTCTTACATAAGAGGACTCTTGGACTCCCAGCAATGTCAACGACCGACCTTGAGGCTACTTCAAAGACTGTGTGTTTAAGGACTGGGAGGAGCTGGG

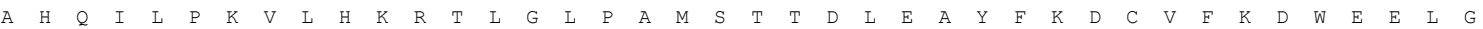

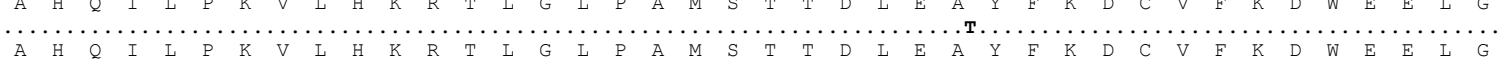

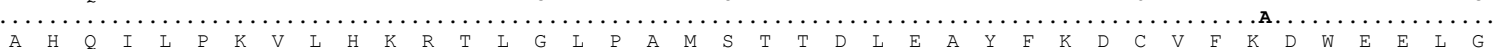

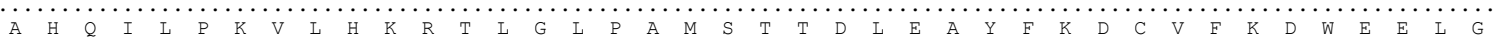

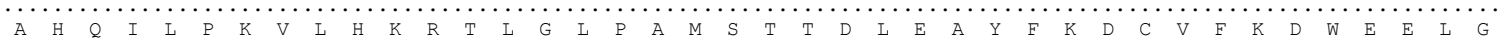

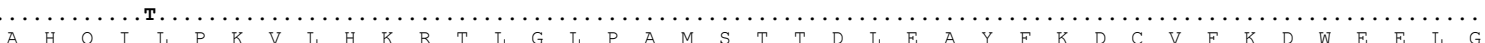

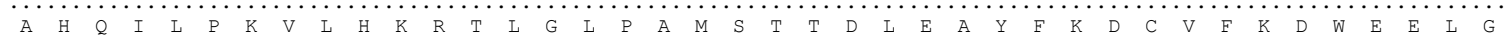
GCCCACCAAATATTGCCCAAGGTCTTACATAAGAGGACTCTTGGACTCTCAGCAATGTCAACGACCGACCTTGAGGCATACTTCAAAGACTGTTTGTTTAAAGACTGGGAGGAGTTGGGG

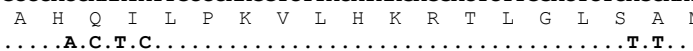

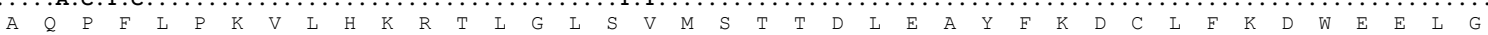

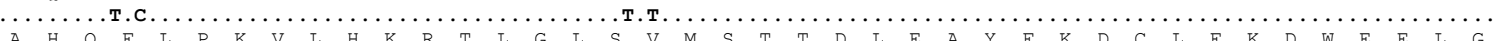
A $\mathrm{H}$ Q $\mathrm{F}$ L $\mathrm{L}$ K $\mathrm{K}$ L

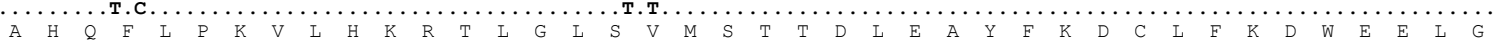

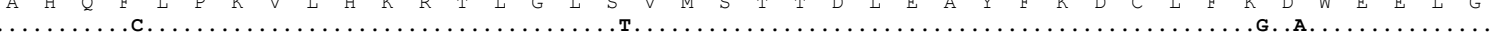

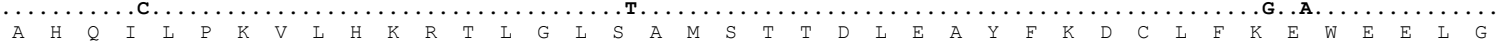
$\ldots$
$A$

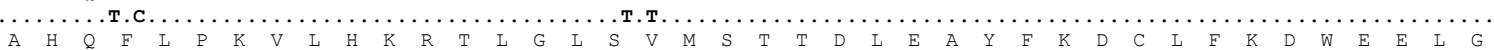

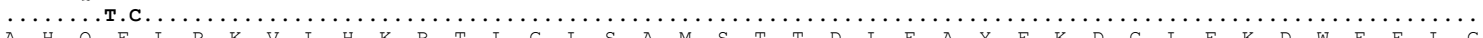

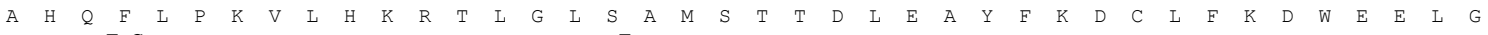

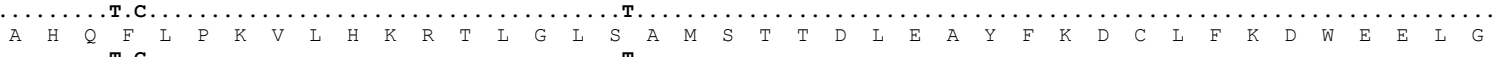

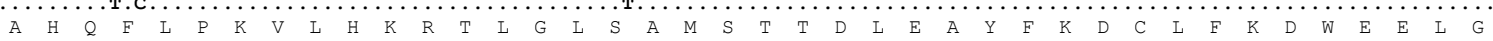

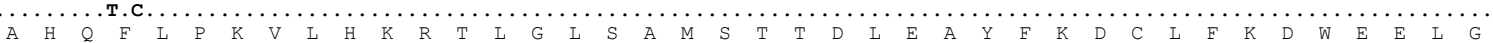

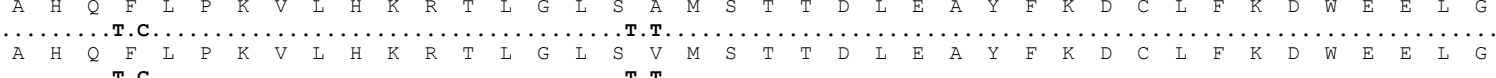

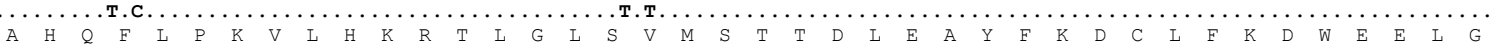

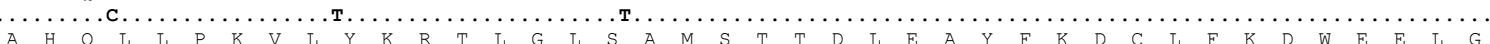

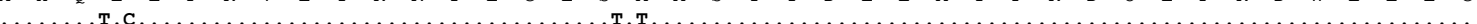

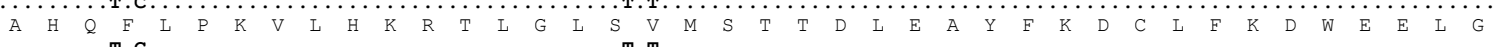

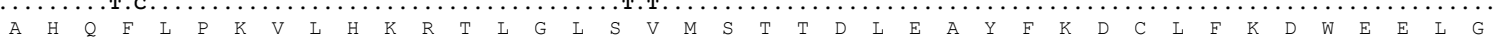

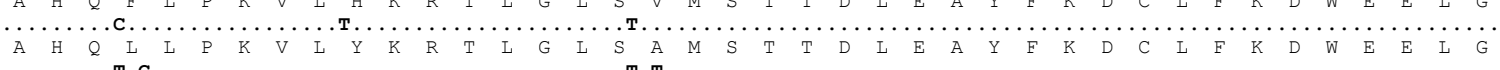

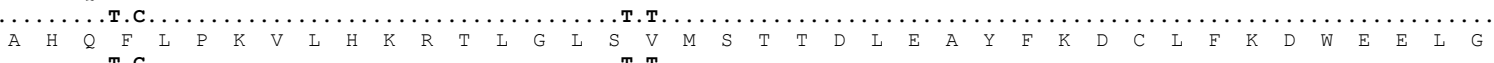

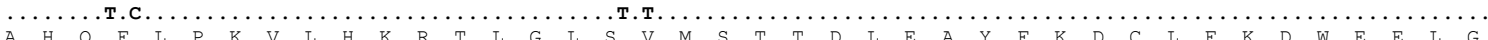

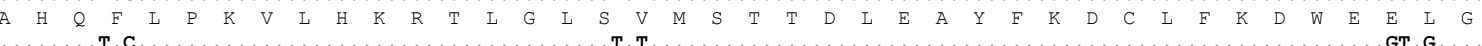

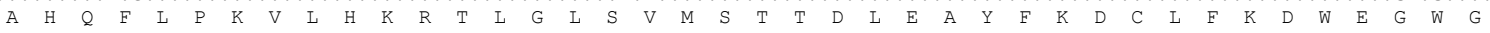
$\underset{A}{A}$\begin{tabular}{l}
$H$ \\
\hline
\end{tabular}

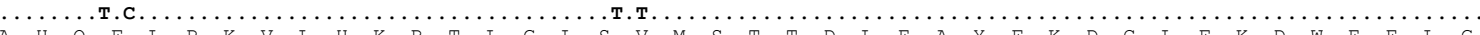

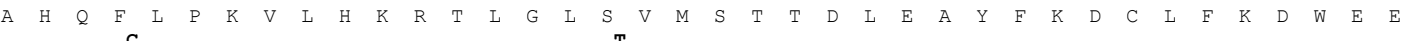

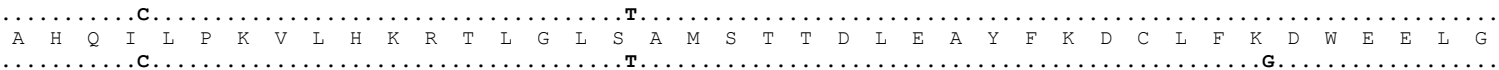

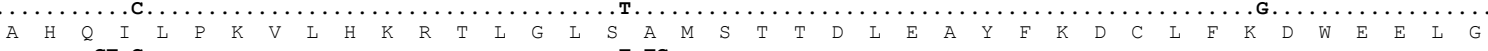

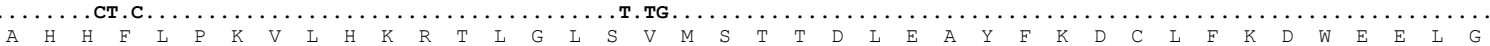

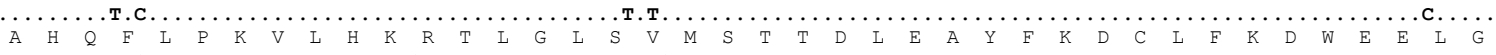

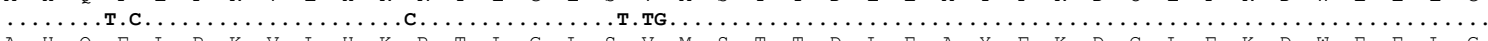

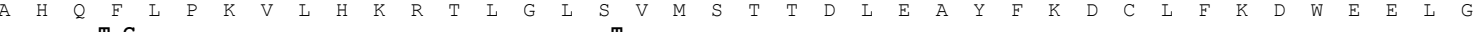

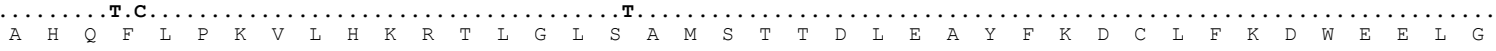

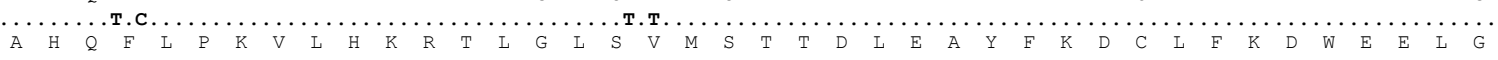




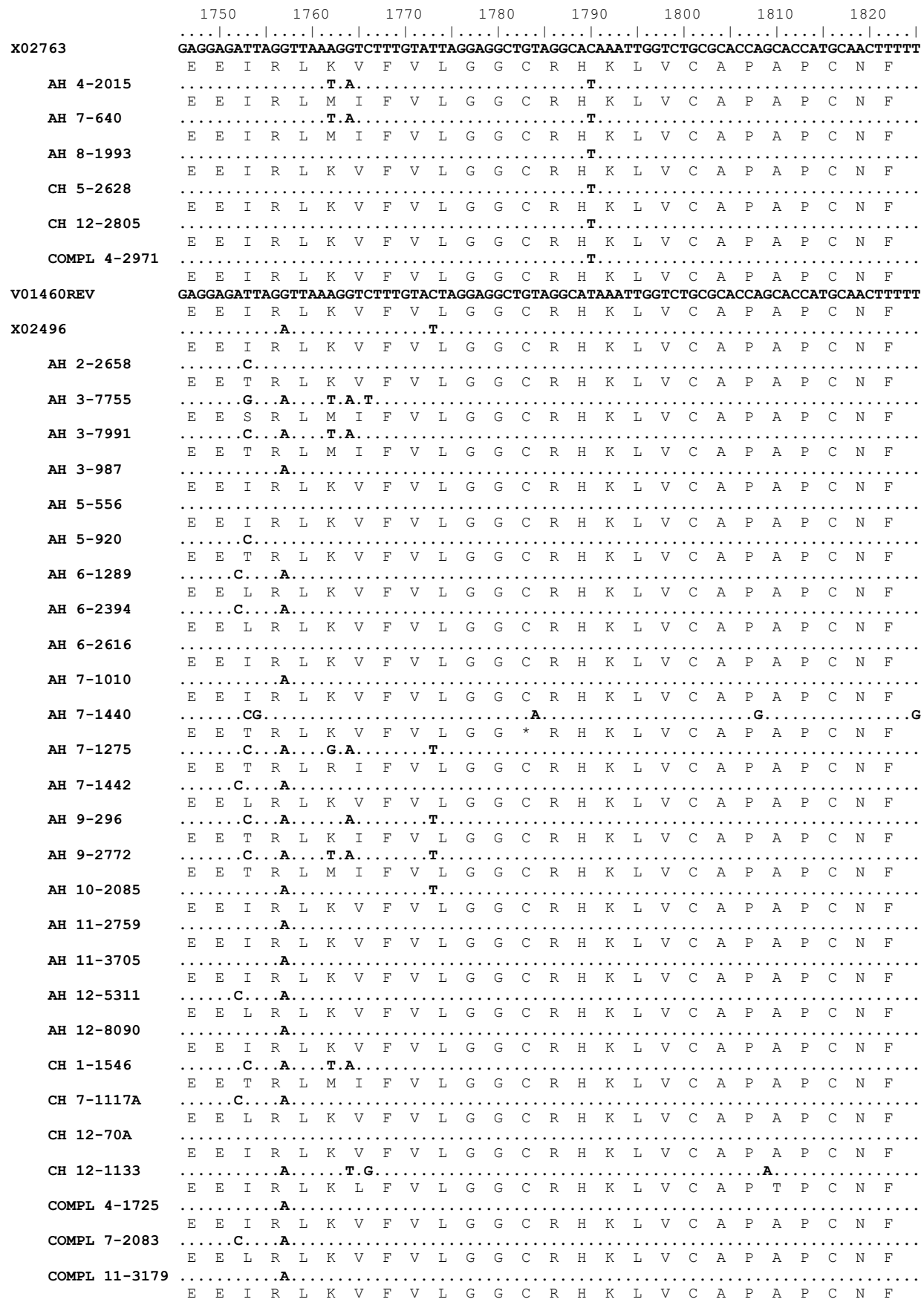

\section{REFERENCES}

[1] Arauz-Ruiz P, Norder H, Robertson BH, Magnius LO. Genotype $\mathrm{H}$ : a new Amerindian genotype of hepatitis $\mathrm{B}$ virus revealed in Central America. J Gen Virol 2002; 83(Pt 8): 2059-73.

[2] Norder H, Courouce AM, Magnius LO. Complete genomes, phylogenetic relatedness, and structural proteins of six strains of the hepatitis B virus, four of which represent two new genotypes. Virology 1994; 198(2): 489-503.

[3] Stuyver L, De GS, Van GC, et al. A new genotype of hepatitis B virus: complete genome and phylogenetic relatedness. J Gen Virol 2000; 81(Pt 1): 67-74.

[4] Kramvis A, Weitzmann L, Owiredu WK, Kew MC. Analysis of the complete genome of subgroup $\mathrm{A}^{\prime}$ hepatitis B virus isolates from South Africa. J Gen Virol 2002; 83(Pt 4): 835-9.

[5] Kurbanov F, Tanaka Y, Fujiwara K, et al. A new subtype (subgenotype) Ac (A3) of hepatitis B virus and recombination between genotypes A and E in Cameroon. J Gen Virol 2005; 86(Pt 7): 2047-56

[6] Norder H, Courouce AM, Coursaget P, et al. Genetic diversity of hepatitis B virus strains derived worldwide: genotypes, subgenotypes, and HBsAg subtypes. Intervirology 2004; 47(6): 289-309.

[7] Magnius LO, Norder H. Subtypes, genotypes and molecular epidemiology of the hepatitis B virus as reflected by sequence variability of the S-gene. Intervirology 1995; 38(1-2): 24-34.

[8] Chu CJ, Lok AS. Clinical significance of hepatitis B virus genotypes. Hepatology 2002; 35(5): 1274-6.

[9] Courouce-Pauty AM, Plancon A, Soulier JP. Distribution of HBsAg subtypes in the world. Vox Sang 1983; 44(4): 197-211.

[10] Pumpen PP, Dishler AV, Kozlovskaia TM, Bychko VV, Gren EI. Cloning of hepatitis B virus DNA in Escherichia coli. Dokl Akad Nauk SSSR 1981; 260(4): 1022-4. 
[11] Pumpen PP, Kozlovskaia TM, Dishler AV, Bychko VV, Kalis I. The comparative mapping of virion and cloned DNA of the hepatitis B virus. Mol Biol (Mosk) 1982; 16(6): 1314-21.

[12] Dumpis U, Kovalova Z, Jansons J, et al. An outbreak of HBV and $\mathrm{HCV}$ infection in a paediatric oncology ward: epidemiological investigations and prevention of further spread. J Med Virol 2003; 69(3): 331-8.

[13] Sominskaya I, Mihailova M, Jansons J, et al. Hepatitis B and C virus variants in long-term immunosuppressed renal transplant patients in Latvia. Intervirology 2005; 48(2-3): 192-200.

[14] Preikschat P, Meisel H, Will H, Gunther S. Hepatitis B virus genomes from long-term immunosuppressed virus carriers are modified by specific mutations in several regions. J Gen Virol 1999; 80 (Pt 10): 2685-91.

[15] Gunther S. Genetic variation in HBV infection: genotypes and mutants. J Clin Virol 2006; 36 (Suppl 1): S3-S11.

[16] Yotsuyanagi H, Hino K, Tomita E, Toyoda J, Yasuda K, Iino S. Precore and core promoter mutations, hepatitis B virus DNA levels and progressive liver injury in chronic hepatitis B. J Hepatol 2002; 37(3): 355-63.

[17] Takahashi K, Ohta Y, Kanai K, et al. Clinical implications of mutations C-to-T1653 and T-to-C/A/G1753 of hepatitis B virus genotype C genome in chronic liver disease. Arch Virol 1999; 144(7): 1299-308.

[18] Lindh M, Hannoun C, Dhillon AP, Norkrans G, Horal P. Core promoter mutations and genotypes in relation to viral replication and liver damage in East Asian hepatitis B virus carriers. J Infect Dis 1999; 179(4): 775-82.

[19] Brunetto MR, Giarin MM, Oliveri F, et al. Wild-type and e antigen-minus hepatitis B viruses and course of chronic hepatitis. Proc Natl Acad Sci USA 1991; 88(10): 4186-90.

[20] Parvez MK, Thakur V, Kazim SN, Guptan RC, Hasnain SE, Sarin SK. Base-pair alterations in the epsilon-lower stem due to a novel double substitution in the precore gene of $\mathrm{HBV}$-e negative variant were recovered by secondary mutations. Virus Genes 2001; 23(3): 315-20.

[21] Chen J, Zhao WD, Wang M, Liu FG, Li JM. Effects of ethanol on HBV replication and gene expression in HBV transgenic mice. Zhonghua Gan Zang Bing Za Zhi 2005; 13(6): 471-2.

[22] Ehata T, Omata M, Yokosuka O, Hosoda K, Ohto M. Variations in codons 84-101 in the core nucleotide sequence correlate with hepatocellular injury in chronic hepatitis B virus infection. J Clin Invest 1992; 89(1): 332-8.

[23] Asahina Y, Enomoto N, Ogura Y, et al. Complete nucleotide sequences of hepatitis $\mathrm{B}$ virus genomes associated with epidemic fulminant hepatitis. J Med Virol 1996; 48(2): 171-8.

[24] Aye TT, Uchida T, Becker SO, Shikata T, Mima S. Completely or nearly identical hepatitis $\mathrm{B}$ virus strains replicate between patients with acute or fulminant hepatitis B and their respective infectious sources. J Med Virol 1994; 42(1): 60-5.

[25] Aye TT, Uchida T, Becker SO, et al. Variations of hepatitis B virus precore/core gene sequence in acute and fulminant hepatitis B. Dig Dis Sci 1994; 39(6): 1281-7.

[26] Asahina Y, Enomoto N, Ogura Y, et al. Sequential changes in fulllength genomes of hepatitis B virus accompanying acute exacerbation of chronic hepatitis B. J Hepatol 1996; 25(6): 787-94.

[27] Hur GM, Lee YI, Suh DJ, Lee JH, Lee YI. Gradual accumulation of mutations in precore core region of HBV in patients with chronic active hepatitis: implications of clustering changes in a small region of the HBV core region. J Med Virol 1996; 48(1): 3846.

[28] Hong P, Ng LF, Ren EC, Chen WN. A cell-based system for hepatitis B virus replication: significance of clinically enhanced viral replication in relation to deletions in viral core promoter. Front Biosci 2005; 10: 2001-4.

[29] Yamashita K, Kagawa S, Matsuoka A, Takarada Y. Detection of hepatitis $\mathrm{B}$ virus by using polymerase chain reaction and nonradioactive DNA probes. II. Identification of mutations in the core gene by PCR-direct sequencing and ASO probe method. Kansenshogaku Zasshi 1996; 70(10): 1072-8.

[30] Bertoletti A, Costanzo A, Chisari FV, et al. Cytotoxic T lymphocyte response to a wild type hepatitis B virus epitope in patients chronically infected by variant viruses carrying substitutions within the epitope. J Exp Med 1994; 180(3): 933-43.
[31] Pumpens P, Grens E, Nassal M. Molecular epidemiology and immunology of hepatitis B virus infection - an update. Intervirology 2002; 45(4-6): 218-32.

[32] Okumura A, Takayanagi M, Aiyama T, et al. Serial analysis of hepatitis B virus core nucleotide sequence of patients with acute exacerbation during chronic infection. J Med Virol 1996; 49(2): 103-9.

[33] Okumura A, Ishikawa T, Yoshioka K, Yuasa R, Fukuzawa Y, Kakumu S. Mutation at codon 130 in hepatitis B virus (HBV) core region increases markedly during acute exacerbation of hepatitis in chronic HBV carriers. J Gastroenterol 2001; 36(2): 103-10.

[34] Takahashi K, Akahane Y, Hino K, Ohta Y, Mishiro S. Hepatitis B virus genomic sequence in the circulation of hepatocellular carcinoma patients: comparative analysis of 40 full-length isolates. Arch Virol 1998; 143(12): 2313-26.

[35] Webster GJ, Reignat S, Maini MK, et al. Incubation phase of acute hepatitis B in man: dynamic of cellular immune mechanisms. Hepatology 2000; 32(5): 1117-24.

[36] Carman WF, Boner W, Fattovich G, et al. Hepatitis B virus core protein mutations are concentrated in $\mathrm{B}$ cell epitopes in progressive disease and in $\mathrm{T}$ helper cell epitopes during clinical remission. J Infect Dis 1997; 175(5): 1093-100.

[37] Preikschat $\mathrm{P}$, Gunther S, Reinhold S, et al. Complex HBV populations with mutations in core promoter, $\mathrm{C}$ gene, and pre-S region are associated with development of cirrhosis in long-term renal transplant recipients. Hepatology 2002; 35(2): 466-77.

[38] Tai PC, Banik D, Lin GI, et al. Novel and frequent mutations of hepatitis B virus coincide with a major histocompatibility complex class I-restricted T-cell epitope of the surface antigen. J Virol 1997; 71(6): 4852-6.

[39] Weinberger KM, Bauer T, Bohm S, Jilg W. High genetic variability of the group-specific a-determinant of hepatitis B virus surface antigen (HBsAg) and the corresponding fragment of the viral polymerase in chronic virus carriers lacking detectable HBsAg in serum. J Gen Virol 2000; 81(Pt 5): 1165-74.

[40] Alexopoulou A, Baltayiannis G, Jammeh S, Waters J, Dourakis SP, Karayiannis P. Hepatitis B surface antigen variant with multiple mutations in the a determinant in an agammaglobulinemic patient. J Clin Microbiol 2004; 42(6): 2861-5.

[41] Chen WN, Oon CJ, Lim GK. Hepatitis B virus surface antigen mutants persist in chronic carriers receiving lamivudine therapy in Singapore. J Antimicrob Chemother 2002; 49(6): 1044-6.

[42] Bielawski KP, Dybikowska A, Lisowska-Charmuszko U, et al. Distribution of HBV genotypes and mutants among hepatitis B infected patients from northern Poland. Int J Mol Med 2004; 14(2): 301-4.

[43] Tallo T, Norder H, Tefanova V, et al. Hepatitis B virus genotype D strains from Estonia share sequence similarity with strains from Siberia and may specify ayw4. J Med Virol 2004; 74(2): 221-7.

[44] Olinger CM, Lazouskaya N, Erimin V, Muller CP. Molecular phylogenie of hepatitis $\mathrm{B}$ and $\mathrm{C}$ in Belarus. The International Meeting on the Molecular Biology of Hepatitis B and C Viruses 2005; pp. 157.

[45] Grannikova SA, Rusakova EV, Budnitskaia PZ, Koroleva VM, Lorinskaia TM. Subtype characteristics of the surface antigen of hepatitis B in different regions of the USSR. Zh Mikrobiol Epidemiol Immunobiol 1977; (10): 97-101.

[46] Abe K, Hayakawa E, Sminov AV, et al. Molecular epidemiology of hepatitis B, C, D and $\mathrm{E}$ viruses among children in Moscow, Russia. J Clin Virol 2004; 30(1): 57-61.

[47] Netesova IG, Swenson PD, Osipova LP, Gubina MA, Posukh OL, Netesov SV. Determination of HBsAg subtypes in Western Siberian part of Russia. J Med Virol 2003; 71(2): 183-7.

[48] Szomor KN, Dencs A, Toth G, et al. Variability of the PreS1/PreS2/S regions of hepatitis B virus in Hungary. Arch Virol 2007; 152(4): 697-704.

[49] Dal MG, Poli A, Croce LS, et al. Hepatitis B virus genotypes, core promoter variants, and precore stop codon variants in patients infected chronically in North-Eastern Italy. J Med Virol 2006; 78(6): 734-40.

[50] De MC, Giambelli C, Tanzi E, et al. High level of genetic heterogeneity in $\mathrm{S}$ and $\mathrm{P}$ genes of genotype $\mathrm{D}$ hepatitis $\mathrm{B}$ virus. Virology 2007; 365(1): 113-24.

[51] Echevarria JM, Avellon A, Magnius LO. Molecular epidemiology of hepatitis B virus in Spain: identification of viral genotypes and 
prediction of antigenic subtypes by limited sequencing. J Med Virol 2005; 76(2): 176-84.

[52] Jardi R, Rodriguez F, Buti M, et al. Mutations in the basic core promoter region of hepatitis $\mathrm{B}$ virus. Relationship with precore variants and HBV genotypes in a Spanish population of HBV carriers. J Hepatol 2004; 40(3): 507-14.
[53] Micalessi MI, De CL, Vranckx R. Hepatitis B virus (HBV) genotyping in Belgian patients with chronic HBV infection. Clin Microbiol Infect 2005; 11(6): 499-501.

[54] van Houdt R, Bruisten SM, Koedijk FD, et al. Molecular epidemiology of acute hepatitis B in the Netherlands in 2004: nationwide survey. J Med Virol 2007; 79(7): 895-901.

Received: June 24, 2010

Revised: August 14,2010

Accepted: December 02, 2010

(C) Sominskaya et al.; Licensee Bentham Open.

This is an open access article licensed under the terms of the Creative Commons Attribution Non-Commercial License (http://creativecommons.org/licenses/by$\mathrm{nc} / 3.0 /$ ), which permits unrestricted, non-commercial use, distribution and reproduction in any medium, provided the work is properly cited. 